\title{
基于[PNP]配体的铬催化剂体系选择性催化乙烯齐聚的研究进展
}

\author{
刘点 ${ }^{a}$ 肖树萌 $b$ 钟向宏*, 曹育才 ${ }^{c}$ 梁胜彪 ${ }^{b}$ \\ 刘振宇 ${ }^{b}$ 叶晓峰 ${ }^{c}$ 沈 安 ${ }^{c}$ 朱红平*, $a$ \\ $\left({ }^{a}\right.$ 厦门大学化学化工学院 固体表面物理化学国家重点实验室 厦门 361005) \\ $\left({ }^{b}\right.$ 中国石油化工股份有限公司茂名分公司 茂名 525021) \\ ( ${ }^{c}$ 上海化工研究院 上海市聚烯烃催化技术重点实验室 上海 200062)
}

\begin{abstract}
摘要 乙烯选择性三聚和四聚催化反应为制备 1-已烯和 1-辛烯提供了重要途径. 在报道和披露的众多结构和组成的催 化剂体系中, 我们选择[PNP]配位骨架的铬催化剂体系, 对其发展和应用进行了综述. 论文以结构与催化性能的关联性 为主线, 阐述 $[\mathrm{PNP}]$ 骨架以及 $\mathrm{N}$ 和 $\mathrm{P}$ 上取代基的电子和立体空间效应对催化性能的影响. 从目前的研究态势来看, [PNP] 配位骨架的铬催化剂体系在乙烯选择性齐聚生产 1-辛烯等线性 $\alpha$-烯烃方面具有发展潜力.
\end{abstract}

关键词 乙烯选择性齐聚; [PNP]配体; 铬催化剂; 1-已烯; 1-辛烯; 催化机理

\section{Advances in Selective Ethylene Oligomerization Based on [PNP]-Ligand Chromium Catalysts}

\author{
Liu, Rui ${ }^{a} \quad$ Xiao, Shumeng ${ }^{b} \quad$ Zhong, Xianghong* ${ }^{*} b$ Cao, Yucai $^{c} \quad$ Liang, Shengbiao ${ }^{b}$ \\ Liu, Zhenyu ${ }^{b} \quad$ Ye, Xiaofeng ${ }^{c} \quad$ Shen, $\mathrm{An}^{c} \quad$ Zhu, Hongping ${ }^{*, a}$ \\ ( ${ }^{a}$ State Key Laboratory of Physical Chemistry of Solid Surfaces, Xiamen University, Xiamen 361005) \\ ( ${ }^{b}$ Maoming Branch R\&D Institute, SINOPEC, Maoming 525011) \\ ( ${ }^{c}$ Shanghai Key Laboratory of Catalysis Technology for Polyolefins, Shanghai Research Institute of Chemical Industry, \\ Shanghai 200062)
}

\begin{abstract}
The catalytic reaction for the selective ethylene trimerization and tetramerization provides a vital route to the production of 1-hexene and 1-octene. Among the numerous catalysts reported with diverse structures and compositions, we select the [PNP]-ligand based chromium catalysts and focus on illustration on their development and application. This contribution includes seven sections as ethylene (selective) oligomerization catalyst systems, [PNP]-ligand based Cr catalyst system, catalytic mechanism, ethylene oligomerization method, catalytic property, and conclusion and prospect, which will cover almost all of the [PNP]-ligand based Cr catalysts so far studied. In this article, we will mainly discuss the influence on the catalytic property owing to the electronic and/or steric characters of the skeleton as well as the substituents at both $\mathrm{N}$ and $\mathrm{P}$ atoms of the [PNP]-ligands. In view of the current advances in this field, this [PNP]-ligand based Cr catalyst system is of potencial in application for the production of LAO mainly as 1-octene.
\end{abstract}

Keywords selective ethylene oligomerization; [PNP] ligand; chromium catalyst; 1-hexene; 1-octene; catalytic mechanism

乙烯齐聚催化是制备线性 $\alpha$-烯烃(LAO)的非常重要 的反应, 也是烯烃聚合催化领域的重要研究内容. 它突 破了传统的 LAO 制备方法, 如蜡裂解、烷烃催化裂解、 烷烃脱氢、煤品抽提、萃取分离、脂肪醇脱氢、烯烃二 聚和歧化、内烯烃异构等, 无论在质量方面还是工业化
流程方面均具长足优势, 目前已经成为最主要的工业生 产方法之一 ${ }^{[1 \sim 3]}$. LAO 是重要的有机化工原料和化学中 间体，用以制备增塑剂、表面活性剂、润滑剂、长链羧 酸、环氧化合物等. LAO 中的很多成分如 1-丁烯、1-已 烯、1-辛烯甚至更高级的 $\alpha$-烯烃是制备高质量和优越性

*E-mail: zhongxh.mmsh@sinopec.com; hpzhu@xmu.edu.cn

Received April 8, 2015; revised May 19, 2015; published online May 26, 2015.

Project Supported by the Key Laboratory of Catalysis Technology for Polyolefins (No. 12DZ2260400), the National Natural Science Foundation of China (No. 21473142), and the Program for Innovative Research Team in Chinese Universities (No. IRT_14R31).

聚烯烃催化技术重点实验室基金(No. 12DZ2260400)、国家自然科学基金(No. 21473142)和教育部创新团队(No. IRT_14R31)资助项目. 
能聚烯烃的不可缺少的共聚单体 ${ }^{[3]}$.

传统的乙烯齐聚催化往往得到多组分的线性 $\alpha$-烯 烃, 呈正态分布特征. 工业上可以根据需要分离单一组 分或几种混合组分加以使用. 乙烯高选择性催化则为生 产单一的特定碳数的 $\alpha$-烯烃提供了重要途径, 对学术界 和工业界都具有积极的意义. 因此, 在乙烯齐聚催化发 展的同时, 乙烯选择性齐聚催化也随之发展.

目前，乙烯选择性齐聚催化报道的有二聚制 1-丁 烯、三聚制 1-己烯以及四聚制 1-辛烯，后两者是近年来 的研究热点. 这些催化集中体现了催化过程中催化剂的 结构调控, 催化剂的结构调控又取决于配体的结构. 乙 烯选择性三聚和四聚催化剂中使用的配体骨架类型有 单齿的 $\mathrm{N}$ 配体、多齿的 $[\mathrm{PNP}],\left[\mathrm{PC}_{n} \mathrm{P}\right],\left[\mathrm{PC}_{n} \mathrm{NC}{ }_{n} \mathrm{P}\right]$, $\left[\mathrm{PC}_{n} \mathrm{PC}_{n} \mathrm{P}\right],\left[\mathrm{SC}_{n} \mathrm{NC}{ }_{n} \mathrm{~S}\right],\left[\mathrm{PC}_{n} \mathrm{NC}_{n} \mathrm{~N}\right],\left[\mathrm{N}_{3}\left(\mathrm{C}_{n}\right)_{3}\right],\left[\mathrm{NC}{ }_{n} \mathrm{~N}\right]$, $\left[\mathrm{PNC}_{n} \mathrm{~N}\right],\left[\mathrm{PNC}_{n} \mathrm{NP}\right]$ 类 ( $n$ 为整数) 以及少部分的茂环及其 衍生类配体 ${ }^{[2]}$. 近年来有为数不多的相关综述报道, 如 2004 年 Morgan ${ }^{[1]} 、 2010$ 年 McGuinness ${ }^{[4]}$, 2011 年 Agapie $^{[5]}$ 和 2012 年 Belov 等 ${ }^{[6]}$ 从基础和应用角度对多种 配体类型的催化剂体系进行了阐述. 此外, 国内的科研 人员也对该领域的工作进行了一些介绍 ${ }^{[7]}$. 本工作围绕 着催化剂的结构与性能的关联性, 主要探讨 [PNP]配位 骨架的配体, 并详细地讨论了由[PNP]配体、铬化物前驱 体以及助剂或直接由 [PNP]配体铬化合物与助剂组成的 体系的催化反应结果, 以期对乙烯选择性三聚和四聚的 催化反应有一定的理解. 从目前的研究态势来看, [PNP] 配位骨架的铬催化剂体系在乙烯选择性齐聚生产 1-辛 烯等 $\alpha$-烯烃方面具有发展潜力.

\section{2 乙烯齐聚和选择性齐聚催化体系}

传统的乙烯齐聚反应使用的催化剂主要有金属铝 系、钛系、镍系、锆系、铁系、钴系等 ${ }^{[1,2]}$. 这些催化体 系的作用原理与经典的 Ziegler-Natta 聚合催化剂的原理 基本一致, 主要遵循 Cossee-Arlman 机理, 即乙烯分子 在催化剂金属中心配位插入线性链增长, 进而发生碳链 的 $\beta$-氢迁移和脱除, 生成 $\alpha$-烯烃. 但在反应过程中, 链 增长速率只是在一定程度上大于链脱除速率(亦称链转 移速率)或与其相当, 与聚合反应的链增长速率远大于 链脱除速率的特点不同. 研究表明, 这是由催化剂的组 成和结构决定的, 同时聚合反应条件如温度、压力、浓 度等也会有很大的影响. 因此, 在乙烯齐聚催化反应中, 碳链的增长有一定限度, 碳数在 $4 \sim 30$ 之间变化. 这样 生成的 $\alpha$-烯烃往往符合 Schulz-Flory 或 Poisson 分布, 其 中处于这些正态分布的峰值表明某些特定的 $\alpha$-烯烃在 所有 $\alpha$-烯烃产物中具有较高的含量. 而这些峰值的位置 变化受链增长因子 $K(K$ 为链增长速率与链增长速率和
链转移速率之和的比值)的影响.

Chevron 公司 ${ }^{[8]}$ 采用传统的 Ziegler 催化剂三烷基铝, 在 $190 \sim 220{ }^{\circ} \mathrm{C}$ 和 $17 \sim 34 \mathrm{MPa}$ 压力下催化乙烯生成不 同偶数碳数的 $\alpha$-烯烃, 呈 Poisson 分布, 低碳 $\mathrm{C}_{4} \sim \mathrm{C}_{8}$ 占 $40 \%$ 以上，其中直链 $\alpha$-烯烃的质量分数达到 $96 \%$. 碳链 增长和碳链置换一步进行(亦称一步法技术). Ineos 公司 同样采用这种催化剂, 但链增长与链置换反应分两步进 行(亦称两步法技术), 得到的 $\alpha$-烯烃中 $\mathrm{C}_{4} \sim \mathrm{C}_{8}$ 约占 $50 \%$, $\mathrm{C}_{20}$ 以上馏分小于 $3 \%, \mathrm{C}_{4} \sim \mathrm{C}_{8}$ 线性 $\alpha$-烯烃的质量分数大 于 $96 \%$, 产品的线性率随碳数的增加而下降, 碳数分布 可通过改变操作条件和产物循环量进行调节, 以符合市 场需求. Shell 公司 ${ }^{[9]}$ 主要使用氮磷配位骨架的金属镍化 合物催化剂, 采用 SHOP (Shell Higher Olefin Process)法, 反应在 $90 \sim 120{ }^{\circ} \mathrm{C}$ 和 9 14 MPa 乙烯压力下进行, 齐聚 产物呈 Flory-Schulz 分布, 烯烃线性率可达 99\%, 低碳 $\mathrm{C}_{6} \sim \mathrm{C}_{10}$ 约占 $52 \%$, 其中 $\alpha$-烯烃的含量高于 $98 \%$. UOP 公司和 UCC 公司 ${ }^{[10]}$ 则采用氯化镍、硽氢化钠及配体 2二苯邻酰基-1-菜磺酸组成的均相催化剂体系, 在 60 $93{ }^{\circ} \mathrm{C}$ 和 7.48 10.2 $\mathrm{MPa}$ 的条件下进行乙烯齐聚, 产品 组成遵循 Schulz-Flory 分布， $\alpha$-烯烃选择性接近 $100 \%$, $\mathrm{C}_{4} \sim \mathrm{C}_{8}$ 线性 $\alpha$-烯烃含量可在 $45 \% \sim 70 \%$ 范围内调节, $K$ 值为 $0.40 \sim 0.67$. 如果要生产 $\mathrm{C}_{4} \sim \mathrm{C}_{10} \alpha$-烯烃, 可通过调 变催化剂将 $K$ 值控制在 0.53 左右; 如果希望得到更多的 $\mathrm{C}_{12} \sim \mathrm{C}_{18} \alpha$-烯烃, 则需调变催化剂使 $K$ 值接近于 0.62 . 上世纪末, Brookhart ${ }^{[11]}$ 、Bennett ${ }^{[12]}$ 和 Gibson ${ }^{[13]}$ 独立地发 现由吡啶二亚胺铁(或钴)与甲基铝氧烷(MAO)组成的催 化剂体系催化乙烯齐聚, 产品为偶数 $\alpha$-烯烃, 选择性超 过 $98 \%$, 符合 Flory-Schulz 分布; 低碳 $\mathrm{C}_{4} \sim \mathrm{C}_{12}$ 约占 $75 \%$, 线性 $\alpha$-烯烃的质量分数大于 $96 \%$. 调节 $K$ 值低至 0.45 , 可生产 $\mathrm{C}_{4} \sim \mathrm{C}_{10} \alpha$-烯烃.

乙烯选择性齐聚的催化反应机理与 Cossee-Arlman 的线性链增长机理不同, 主要遵循金属环化机理 ${ }^{[2 \sim 4]}$. 在这种反应机理的作用下, $\mathrm{MC}_{n}$ 环的形成是乙烯选择性 齐聚反应的结构关键点, 本质上又决定于 $\mathrm{M}$ 及其周边 的配位环境, 外在的条件如温度、压力以及浓度等也起 着重要的影响.

乙烯选择性二聚制备 1-丁烯是最为简单的齐聚反 应 $^{[4,14]}$. 追溯源头, 该反应过程由 Ziegler 和 Martin 于 1960 年发现, 催化剂由烷氧基钛(或烷氧基锆)与三乙基 铝组成 ${ }^{[15]} .20$ 年后, Chauvin 等 ${ }^{[16]}$ 发展了这一催化过程, 称为生产 1-丁烯的 “Alphabutol Process”。最好的催化 剂是 $\mathrm{Ti}(\mathrm{OBu})_{4} / \mathrm{AlEt}_{3}$, 乙烯分子的转换频率达 $1 \times 10^{6}$ $\mathrm{h}^{-1}, 1$-丁烯的选择性达 93\%.

乙烯选择性三聚最早由美国联碳公司的 Manyik, Walker 和 Wilson 于 1964 年在研究乙烯聚合的过程中发 
现, 催化剂由有部分水解的三异丁基铝和三(2-乙基己 酸)铬组成 ${ }^{[17]}$. 在催化反应中, 部分乙烯三聚生成 1-己 烯, 并和乙烯共聚生成含丁基侧链的聚合物. Phillips 公 司在此基础上引入吡咯基配体, 并调变助剂, 形成了由 2-乙基己酸铬、2,5-二甲基吡咯、三乙基铝和二乙基氯 化铝组成的催化剂体系, 在环己烷溶剂中 $115{ }^{\circ} \mathrm{C}$ 和 10 $\mathrm{MPa}$ 压力下高选择性催化乙烯三聚, 生成 1-己烯高达 $93 \%$, 活性达到 $1.56 \times 10^{5} \mathrm{~g} /(\mathrm{g} \mathrm{Cr} \bullet \mathrm{h})^{[18]} .2002$ 年，英国石 油(BP)公司 Wass 等 ${ }^{[19]}$ 发现由三氯化铬、磷氮磷(PNP) 配体、甲基铝氧烷组成的体系在甲苯溶剂中 $80{ }^{\circ} \mathrm{C}$ 和 2.0 $\mathrm{MPa}$ 压力下也高选择性催化乙烯三聚, 生成 1-己烯近 $90 \%$, 活性达到 $1.03 \times 10^{6} \mathrm{~g} /(\mathrm{g} \mathrm{Cr} \bullet \mathrm{h})$, 反应条件比较温 和.

2004 年, Sasol 技术公司的研究人员在英国石油公 司的研究基础上, 将磷氮磷配体中磷原子上芳基取代基 由甲氧基变换为氢或烷基基团, 其与三氯化铬和甲基铝 氧烷组成的体系在甲苯溶剂中 $65{ }^{\circ} \mathrm{C}$ 和 $3.0 \mathrm{MPa}$ 压力下 或 $45{ }^{\circ} \mathrm{C}$ 和 $4.5 \mathrm{MPa}$ 压力下选择性催化乙烯四聚, 生成 1-辛烯为主导产物，达 $44.0 \% \sim 67.5 \%$, 另含 $16.6 \%$ $32.7 \%$ 的 $\mathrm{C}_{6}$ 烯烃, 其中 1-已烯的选择性低于 $80 \%$, 活性 在 $8.05 \times 10^{3} \sim 4.36 \times 10^{4} \mathrm{~g} /(\mathrm{g} \mathrm{Cr} \cdot \mathrm{h})$ 范围内变化 ${ }^{[20]} .2010$ 年 Gambarotta 等 ${ }^{[21]}$ 报道采用 N-桥连的双吡啶配体铬化 合物 $\left[\left(2-\mathrm{C}_{5} \mathrm{H}_{4} \mathrm{~N}\right)_{2} \mathrm{NR}\right] \mathrm{CrCl}_{3}$ (THF) $\left(\mathrm{R}=\mathrm{Me}, \mathrm{CH}_{2} \mathrm{CMe}_{3}\right.$, $\mathrm{CH}_{2} \mathrm{SiMe}_{3}, \mathrm{C}_{16} \mathrm{H}_{33}$, benzyl, $\left.\mathrm{C}_{6} \mathrm{H}_{3} \mathrm{Si}(\mathrm{OEt})_{3}, \mathrm{C}_{4} \mathrm{H}_{8} \mathrm{OEt}\right)$ 在 MAO 的活化下, 于甲苯溶剂中 $35 \sim 110{ }^{\circ} \mathrm{C}$ 和 $4.0 \mathrm{MPa}$ 压力下催化乙烯反应, 产物基本上是 1-辛烯, 但产率很 低 $(2.2 \% \sim 13.5 \%)$, 大量的产物是聚乙烯蜡. 到目前为 止，乙烯五聚甚至更高级的选择性齐聚反应未见报道.

仔细研究这些结果可以发现, 催化剂组成中的金属 $\mathrm{M}$ 以及其周边辅助配体或多或少存在着差异. 也正是这 种差异, 允许化学家们进行更细致的探究, 并发现和创 造新的催化反应体系.

\section{3 [PNP]配位骨架的铬催化剂体系}

\section{1 [PNP]配体及其合成}

前面已经述及, [PNP]配位骨架的铬化合物催化剂 自 2002 年发现以来在乙烯选择性齐聚制备 1-已烯和 1辛烯方面展现了独特的结构调控作用 ${ }^{[19]}$. 这种独特的 调控作用本质上源于 [PNP]配体. [PNP]配体是指氮原子 上键联两个磷原子形成 $\mathrm{P}, \mathrm{N}, \mathrm{P}-$ 骨架的有机化合物, 氮原 子上可键联一个氢原子或一个有机基团 $\left(\mathrm{R}^{1}\right)$, 每个磷原 子可以独立地键联两个原子、两个基团或一个原子和一 个基团 $\left(\mathrm{R}^{2}, \mathrm{R}^{3}, \mathrm{R}^{4}\right.$ 和 $\mathrm{R}^{5}$ )(图 1). [PNP]配体的合成取决于 氮磷键的构筑方法, 但随氮和磷上取代基的变化会有所 不同. 含不同取代基的氮源和磷源在亲核(或亲电)和立
体空间作用方面有着差异性.<smiles>[R]PN([R])P[R7]</smiles>

图 1 [PNP]配体的结构式

Figure 1 The concise structure of PNP-ligand

构筑氮磷键的常用方法是三卤化磷与胺类分子作 用脱去卤化氢的反应. 早在 20 世纪 60 年代末和 70 年代 初, Nixon 等 ${ }^{[22]}$ 使用三氯化磷作为磷源, 伯胺或其盐酸 盐为氮源, 通过脱氯化氢的方法合成具有 $\mathrm{RN}\left(\mathrm{PCl}_{2}\right)_{2}$ 结 构式的化合物(Eqs. 1 和 2). 该合成反应需要控制好反应 物的计量比, 否则会生成环状的 $(\mathrm{NP})_{n}$ 类化合物. 如果 氮源使用硅基取代的前驱体 $\mathrm{MeN}\left(\mathrm{SiMe}_{3}\right)_{2}$, 反应则经过 脱 $\mathrm{Me}_{3} \mathrm{SiCl}$ 生成 $\mathrm{RN}\left(\mathrm{PCl}_{2}\right)_{2}(\mathrm{Eq} .3)^{[23]}$. 从本文讨论的乙 烯齐聚催化的角度考虑，磷原子键联氯原子的化合物 $\mathrm{RN}\left(\mathrm{PCl}_{2}\right)_{2}$ 是不适合用作目标配体. 但是这些氯原子可 以进一步进行基团变换反应，如 Eq. 5 , 其与 $\mathrm{F}^{-}$或 $\mathrm{OR}^{\prime}$ 发生基团交换生成需要的目标化合物 ${ }^{[24]}$. Bercaw 等 ${ }^{[25]}$ 也正是采用这样的化合物制备了特殊磷取代基的 [PNP] 配体(Scheme 1).
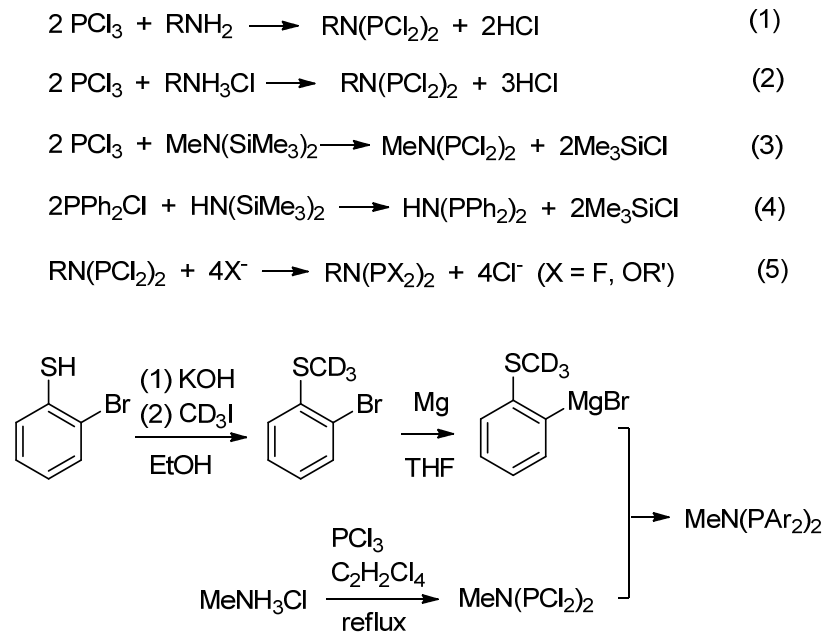

图式 1 多步 Metathesis 反应合成 $\mathrm{MeN}\left(\mathrm{PAr}_{2}\right)_{2}$

Scheme 1 Multiple-step metathesis synthesis of $\mathrm{MeN}\left(\mathrm{PAr}_{2}\right)_{2}$

相应地, 以 $\mathrm{PPh}_{2} \mathrm{Cl}$ 作为磷源和 $\mathrm{HN}\left(\mathrm{SiMe}_{3}\right)_{2}$ 为氮源, 两者反应脱去 $\mathrm{Me}_{3} \mathrm{SiCl}$ 生成 $\mathrm{HN}\left(\mathrm{PPh}_{2}\right)_{2}(\mathrm{Eq} .4)^{[26]}$. 该化 合物是很好的催化剂配体，同时也可以用作前驱体. 如 三甲基硅基取代的配体 $\mathrm{Ph}_{2} \mathrm{PN}\left(\mathrm{SiMe}_{3}\right) \mathrm{PPh}_{2}$ 则是以 $\mathrm{HN}\left(\mathrm{PPh}_{2}\right)_{2}$ 为前驱体通过锂化脱质子再进行无机盐消除 法制备(Eq. 6) ${ }^{[27]}$.

一些文献也报道 $\mathrm{PCl}_{3}$ 与 $\mathrm{PCl}_{2}(\mathrm{NH} t \mathrm{Bu})$ 反应制备 $t-\mathrm{BuN}\left(\mathrm{PCl}_{2}\right)_{2}$, 其中使用有机碱 $\mathrm{Et}_{3} \mathrm{~N}$ 为 $\mathrm{HCl}$ 的成盐体, 从而从有机溶剂中析出, 促进反应的进行以及产物的相 


$$
\mathrm{HN}\left(\mathrm{PPh}_{2}\right)_{2} \stackrel{\stackrel{(1) n \mathrm{BuLi}}{(2) \mathrm{Me}_{3} \mathrm{SiCl}}}{\mathrm{THF}} \mathrm{Ph}_{2} \mathrm{PN}\left(\mathrm{SiMe}_{3}\right) \mathrm{PPh}_{2}
$$

分离 $\left(\right.$ Eq. 7) ${ }^{[28]}$. 该反应可以看成是 $\mathrm{PCl}_{3}$ 与 $\mathrm{RNH}_{2}$ (或 $\mathrm{RNH}_{3} \mathrm{Cl}$ ) 合成 $\mathrm{RN}\left(\mathrm{PCl}_{2}\right)_{2}$ 的分步骤, $\mathrm{PCl}_{2}(\mathrm{NHR})$ 是中间产 物. 然而, 如果使用 $\mathrm{R}_{2}{ }_{2} \mathrm{P}(\mathrm{NHR})$ 为前驱体, 其与 $\mathrm{PR}_{2}{ }_{2} \mathrm{Cl}$ 反应则是制备不对称含磷取代基的 [PNP]配体的有效方 法. $\mathrm{Ph}_{2} \mathrm{PN}(i \mathrm{Pr}) \mathrm{P}\left(\mathrm{O}_{2} \mathrm{C}_{6} \mathrm{H}_{4}\right)$ 的合成路线见 Eq. $8^{[29]}$, Scheme 2 展示了变换较为复杂的合成路线 ${ }^{[30]}$.

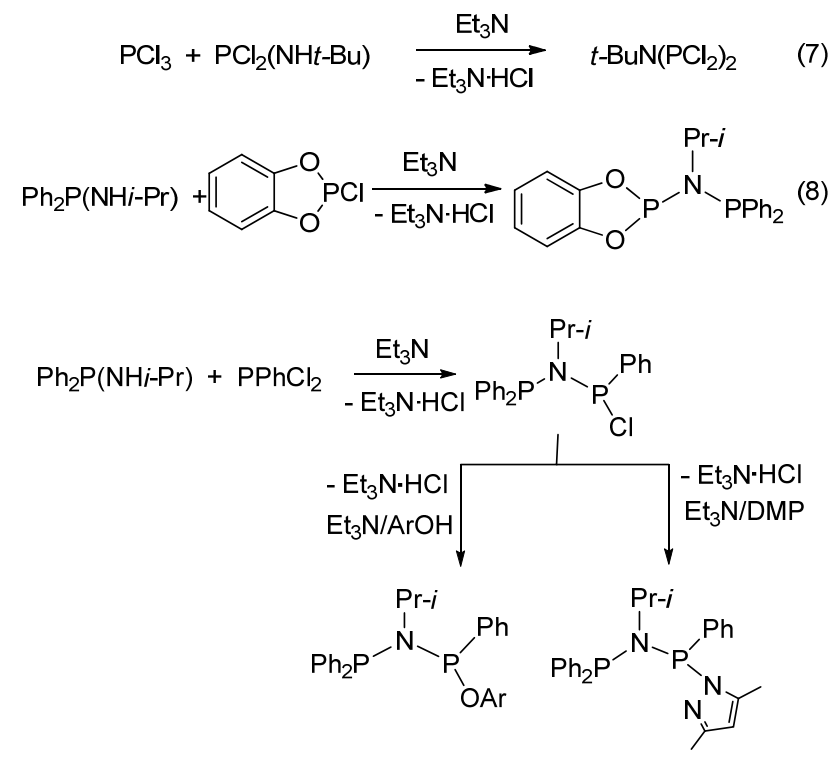

图式 2 制备不对称 [PNP]配体的有效方法

Scheme 2 The effective way to asymmetric PNP-ligand

总的来说, $[\mathrm{PNP}]$ 配体中氮和磷原子上的取代基主 要是有机基团(氮原子上的基团也可以是氢原子). 因此, 合成这类配体比较便捷的方法可如 Eq. 1 所示, 即以伯 胺为氮源和二取代的氯化膦为磷源在有机碱的存在下 脱氯化氢的反应, 其中磷源和氮源化合物的物质的量比 为 $2: 1$ (Eq. 7, $\mathrm{R}^{\prime}$ 与 $\mathrm{R}^{\prime \prime}$ 可相同或相异).

$$
2 \mathrm{R}^{\prime} \mathrm{R} " \mathrm{PCl}+\mathrm{RNH}_{2} \frac{2 \mathrm{Et}_{3} \mathrm{~N}}{-2 \mathrm{Et}_{3} \mathrm{~N} \cdot \mathrm{HCl}} \mathrm{RN}\left(\mathrm{PR}^{\prime} \mathrm{R}^{\prime \prime}\right)_{2}
$$

但是，一些基团较大的如叔丁基、金刚烷基、新戊 基等取代的伯胺, 采用 Eq. 9 所示的方法不能得到目标 产物, 往往只停留在中间物 $\mathrm{R}^{\prime} \mathrm{R}^{\prime \prime} \mathrm{P}(\mathrm{NHR})$, 相应的机理 推测和谱学分析认为按照这样的计量比, 反应最终会重 排成化合物 $\mathrm{Ph}_{2} \mathrm{P}(\mathrm{Ph})_{2} \mathrm{P}=\mathrm{NR}^{[31]}$. Maumela 等 ${ }^{[32]}$ 采用磷源 $\mathrm{R}^{\prime} \mathrm{R}^{\prime \prime} \mathrm{PCl}$ 和有机碱均过量的方法解决了这方面的问题. 最近, 我们 ${ }^{[33]}$ 制备了一种基于四氢康胺的 [PNP]配体, 发现不论使用计量比还是过量法, 均得不到目标化合 物. 采用分步形成胺基锂盐的盐消除反应, 则可以成功
(1) $n$-BuLi

(2) $\mathrm{PPh}_{2} \mathrm{Cl}$

$\mathrm{C}_{4} \mathrm{H}_{7} \mathrm{OCH}_{2} \mathrm{NH}_{2} \underset{n \text {-hexane }}{\stackrel{(2)}{\longrightarrow}} \mathrm{Ph}_{2} \mathrm{PNH}\left(\mathrm{CH}_{2} \mathrm{OC}_{4} \mathrm{H}_{7}\right)$

(1) $n$-BuLi

(2) $\mathrm{PPh}_{2} \mathrm{Cl}$

$\mathrm{Ph}_{2} \mathrm{PN}\left(\mathrm{CH}_{2} \mathrm{OC}_{4} \mathrm{H}_{7}\right) \mathrm{PPh}_{2}$

图式 3 逐步锂化法制备[PNP]配体

Scheme 3 Stepwise lithiation to prepare the PNP-ligand

地得到该类配体化合物(Scheme 3). 尽管该方法可能会 产生类似重排的产物或副产物，但是这是合成[PNP]配 体的另一种重要的行之有效的方法.

\section{2 [PNP]配体铬化合物及其合成}

合成 [P N P ] 配体铬化合物的铬源主要有 $\mathrm{CrCl}_{3}$ (THF) $)_{3}, \mathrm{Cr}(\mathrm{acac})_{3}, \mathrm{CrCl}_{2}$ (tolyl)(THF) $)_{3}, \mathrm{CrCl}_{2}$, $\mathrm{CrCl}_{2}(\mathrm{THF})_{2}, \mathrm{Cr}(\mathrm{CO})_{6}, \mathrm{Cr}(\mathrm{EH})_{3}\left(\mathrm{EH}=\mathrm{OOCCH}(\mathrm{Et}) \mathrm{CH}_{2}-\right.$ $\left.\mathrm{CH}_{2} \mathrm{CH}_{2} \mathrm{CH}_{3}\right), \mathrm{CrCl}_{2} \mathrm{Me}(\mathrm{THF})_{3}, \mathrm{Cr}(\mathrm{Ph})_{3}(\mathrm{THF})_{3}$. 方法相对 简单, 即配体和铬源化合物以等物质的量在有机溶剂中 反应. [PNP]配体与 $\mathrm{CrCl}_{3}(\mathrm{THF})_{3}$ 或 $\mathrm{Cr}(\mathrm{CO})_{6}$ 的反应通常 需要加热, 而与 $\mathrm{Cr}(\mathrm{Ph})_{3}(\mathrm{THF})_{3}$ 的反应在室温下进行即 可. 有文献报道[PNP]配体与 $\mathrm{CrCl}_{2}$ (tolyl)(THF) $)_{3}$ 和 $\mathrm{CrCl}_{2}$ 不反应 ${ }^{[39]}$. 与其它铬源如 $\mathrm{Cr}(\mathrm{acac})_{3}$ 和 $\mathrm{Cr}(\mathrm{EH})_{3}$ 反应生成 相应的铬化合物也未见报道. 但是, 当这些混合物在助 剂烷基铝的作用下会发生反应，并形成活性中心，其中 的反应过程比较复杂. 尽管化学家们对 [PNP]配体铬化 合物催化乙烯齐聚的机理有一定的通识，但活性中心的 模型结构物至今为止仍然未知. 在很多情况下, [PNP] 配体与铬源化合物混合，在助剂的作用下直接用于乙烯 的齐聚反应. 有报道称在聚合技术保证的前提下, 预先 制备好的和原位形成的[PNP]配体铬化合物的催化结果 差异不大 ${ }^{[20]}$.

Scheme 4 是化合物 $\left[\left(\mathrm{R}^{2} \mathrm{R}^{3} \mathrm{PN}\left(\mathrm{R}^{1}\right) \mathrm{PR}^{4} \mathrm{R}^{5}\right) \mathrm{CrCl}_{2}(\mu\right.$ $\mathrm{Cl})]_{2}$ 的合成方法. 该化合物固体状态以二聚体的形式存 在. 在电子给体分子的作用下可解聚成单体. 这些化合 物具顺磁性, 往往通过红外(IR)和顺磁共振(EPR)谱学 以及熔点、质谱(MS)和元素分析进行表征. 明确的分子 结构则需要经 X 射线单晶衍射结晶学确定. 2002 年, 英 国石油公司 (BP) 的 Wass 等 ${ }^{[19]}$ 报道 [PNP] 配体/ $\mathrm{CrCl}_{3}(\mathrm{THF})_{3} / \mathrm{MAO}(\mathrm{MAO}$, 甲基铝氧烷)催化体系用于乙 烯三聚反应，其中的配体铬化合物则是原位形成. 第一 例这种具有单晶结构的化合物由南非 Sasol 技术公司的 研究团队于 2004 年报道 ${ }^{[20]}$. [PNP]配体和 $\mathrm{CrCl}_{3}(\mathrm{THF})_{3}$ 在甲苯溶剂中 $80{ }^{\circ} \mathrm{C}$ 下加热 $12 \mathrm{~h}$ 得到蓝色的固体. 颜色 与原料相比([PNP]配体通常呈白色, $\mathrm{CrCl}_{3}(\mathrm{THF})_{3}$ 呈紫红 色)差异较大，可用于判断产物的形成. 
<smiles>[R]N(P([R])[R7])P([R])[R]</smiles>

$R, R^{1} \sim R^{5}$ : organic group

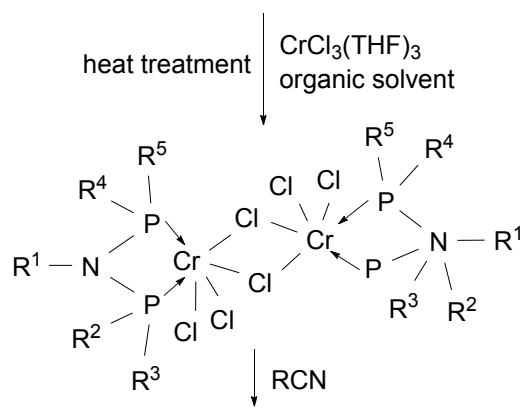<smiles>[R]N[C@]1(Cl)[P+]([R])([R])N([R])[PH]1([R7])Cl</smiles>

图式 $4\left[\left(\mathrm{R}^{2} \mathrm{R}^{3} \mathrm{PN}\left(\mathrm{R}^{1}\right) \mathrm{PR}^{4} \mathrm{R}^{5}\right) \mathrm{CrCl}_{2}(\mu-\mathrm{Cl})\right]_{2}$ 的合成及其解聚 Scheme 4 Synthesis and dissociation of $\left[\left(R^{2} R^{3} P N\left(R^{1}\right) P R^{4} R^{5}\right)\right.$ $\left.\mathrm{CrCl}_{2}(\mu-\mathrm{Cl})\right]_{2}$

上述二聚体化合物的单晶结构揭示 $\mathrm{Cr}-\mathrm{P}$ 键长在 $2.4 \sim 2.6 \AA$ 之间, $\mathrm{P}-\mathrm{N}-\mathrm{P}$ 的键角在 $106^{\circ}$ 左右, 而 $\mathrm{P}-$ $\mathrm{Cr}-\mathrm{P}$ 的键角在 $66^{\circ}$ 左右, 比较窄. 铬中心由于较窄的 $\mathrm{P}-\mathrm{Cr}-\mathrm{P}$ 角呈畸变的八面体配位几何, 其中铬中心有两 个氯原子端基键联, 并被一个氯原子桥联。化合物 $\left[\left(\mathrm{R}^{2} \mathrm{R}^{3} \mathrm{PN}\left(\mathrm{R}^{1}\right) \mathrm{PR}^{4} \mathrm{R}^{5}\right) \mathrm{CrCl}_{2}(\mu-\mathrm{Cl})\right]_{2}$ 呈二聚体结构, 本质上 可以归结为铬中心的 Lewis 酸性, 这种酸性也因多个具 吸电子性的氯原子基团的键联得到增强. 乙腈分子配位 的单核化合物中铬中心仍保持八面体配位几何, 相应的 $\mathrm{Cr}-\mathrm{P}$ 键长和 $\mathrm{P}-\mathrm{N}-\mathrm{P}$ 与 $\mathrm{P}-\mathrm{Cr}-\mathrm{P}$ 键角变化甚微.

[PNP]配体中磷原子上芳基邻位甲氧或甲硫取代基 容易与铬中心配位 ${ }^{[34,35]}$. Scheme 5 中化合物 $\left[\mathrm{Ar}_{2} \mathrm{PN}(\mathrm{Me})-\right.$ $\left.\mathrm{PAr}_{2}\right] \mathrm{CrPh}_{3} \mathrm{~A}$ 由于甲氧基的配位呈单核结构. 相应的甲 硫基配位能力更强, 会 “挤掉” 其中一个磷配位形成化 合物 B. 值得注意的是, 当使用电子给体分子如四氢呋 喃(THF)为反应溶剂分子时, 反应处于一个严格意义上 的可逆过程, 这点可以通过变温的 ${ }^{2} \mathrm{H}$ 核磁共振谱证明. 这种配位模式对铬中心的官能基转换几乎没有影响，如 Eq. 10 所示, 化合物 $\left[\mathrm{Ar}_{2} \mathrm{PN}(\mathrm{Me}) \mathrm{PAr}_{2}\right] \mathrm{CrCl}_{3}$ (C), 下面会 提及) 与 $\mathbf{A}$ 反应生成 $\left[\mathrm{Ar}_{2} \mathrm{PN}(\mathrm{Me}) \mathrm{PAr}_{2}\right] \mathrm{CrClPh}_{2}(\mathbf{D})$. 进一 步使用 $\left[\mathrm{H}\left(\mathrm{OEt}_{2}\right)_{2}\right]^{+}\left\{\mathrm{B}\left[\mathrm{C}_{6} \mathrm{H}_{3}\left(\mathrm{CF}_{3}\right)_{2}\right]_{4}\right\}^{-}$或 $\mathrm{Na}^{+}\left\{\mathrm{B}\left[\mathrm{C}_{6} \mathrm{H}_{3}-\right.\right.$ $\left.\left.\left(\mathrm{CF}_{3}\right)_{2}\right]_{4}\right\}^{-}$拔掉铬中心的一个官能基，相应的会有另一 个甲氧取代基 “补充” 配位，形成 $\left[\left(\mathrm{Ar}_{2} \mathrm{PN}(\mathrm{Me}) \mathrm{PAr}_{2}\right)-\right.$

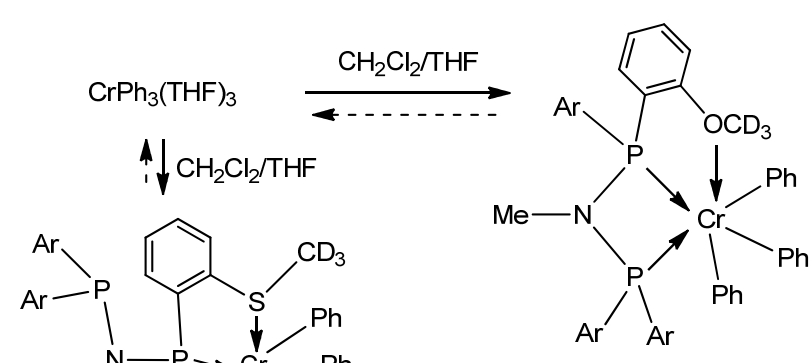

A $\mathrm{Ar}=0-\mathrm{OMeC} 6 \mathrm{H} 4$
图式 $5\left[\mathrm{Ar}_{2} \mathrm{PN}(\mathrm{Me}) \mathrm{PAr}_{2}\right] \mathrm{CrPh}_{3}$ 的合成 Scheme 5 Synthesis of $\left[\mathrm{Ar}_{2} \mathrm{PN}(\mathrm{Me}) \mathrm{PAr}_{2}\right] \mathrm{CrPh}_{3}$

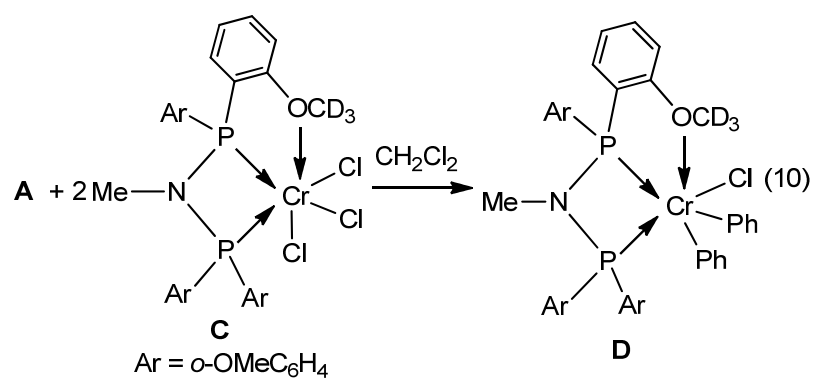

$\left.\mathrm{CrPh}_{2}\right]^{+}\left\{\mathrm{B}\left[\mathrm{C}_{6} \mathrm{H}_{3}\left(\mathrm{CF}_{3}\right)_{2}\right]_{4}\right\}^{-}(\mathbf{E})(\text { Scheme } 7)^{[34]}$. 单晶结构 揭示 $\mathrm{Cr}-\mathrm{O}$ 键长在 $2.25 \sim 2.45 \AA$ 间, 呈共价键, 反映了 铬中心与甲氧基的强的键合作用.

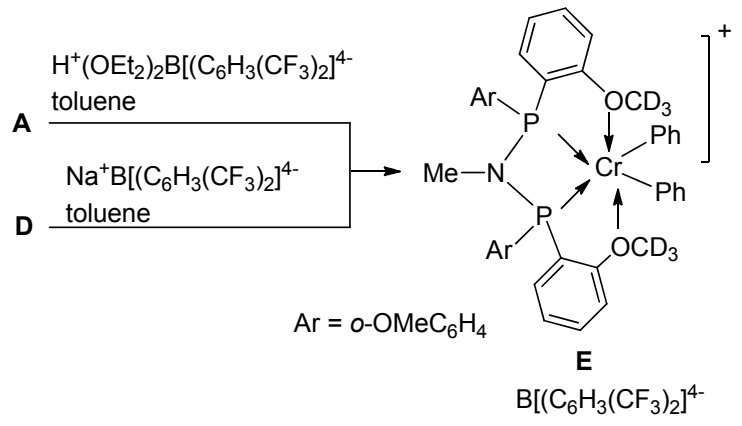

图式 $6\left[\mathrm{Ar}_{2} \mathrm{PN}(\mathrm{Me}) \mathrm{PAr}_{2}\right] \mathrm{CrPh}_{2} \mathrm{Cl}$ 的合成

Scheme 6 Synthesis of $\left[\mathrm{Ar}_{2} \mathrm{PN}(\mathrm{Me}) \mathrm{PAr}_{2}\right] \mathrm{CrPh}_{2} \mathrm{Cl}$

[PNP] 配体与 $\mathrm{Cr}(\mathrm{CO})_{6}$ 在有机溶剂中加热反应一定 时间脱去两个 $\mathrm{CO}$ 分子生成化合物 $\left[\mathrm{Ar}_{2} \mathrm{PN}(\mathrm{R}) \mathrm{PAr}_{2}\right] \mathrm{Cr}-$ $(\mathrm{CO})_{4}(\mathbf{F})$. 化合物 $\mathbf{F}$ 与 $\mathrm{Ag}\left\{\mathrm{Al}\left[\mathrm{OC}\left(\mathrm{CF}_{3}\right)_{3}\right]_{3}\right\}$ 发生单电子还 原反应生成 $\left.\left[\mathrm{Ar}_{2} \mathrm{PN}(\mathrm{R}) \mathrm{PAr}_{2}\right) \mathrm{Cr}(\mathrm{CO})_{4}\right]^{+}\{\mathrm{Al}[\mathrm{OC}-$ $\left.\left.\left(\mathrm{CF}_{3}\right)_{3}\right]_{4}\right\}^{-}(\mathbf{G})($ Scheme 7). 同时, 化合物 $\mathbf{F}$ 与卤素及其 衍生物反应生成三价态铬化合物 $\left[\mathrm{Ar}_{2} \mathrm{PN}(\mathrm{R}) \mathrm{PAr}_{2}\right] \mathrm{CrX}_{3}$ $(\mathbf{H}, \mathrm{X}=\mathrm{Cl}, \mathrm{Br}, \mathrm{I}), \mathbf{H}$ 与联苯基格氏试剂反应生成化合物 $\left[\mathrm{Ar}_{2} \mathrm{PN}(\mathrm{R}) \mathrm{PAr}_{2}\right] \mathrm{CrBr}$ (biphenyl) (I) (Scheme 8$)^{[35]}$. 化 

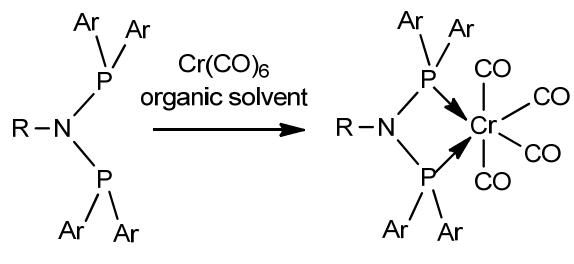

R: organic group Ar: aryl group
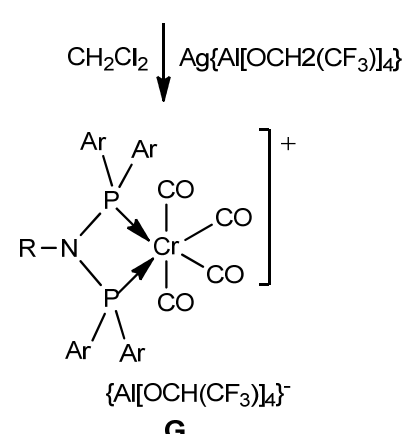

图式 $\left.7\left[\mathrm{Ar}_{2} \mathrm{PN}(\mathrm{R}) \mathrm{PAr}_{2}\right)\right] \mathrm{Cr}(\mathrm{CO})_{4}$ 的合成及其单电子还原反应 Scheme 7 Synthesis and single-electron reduction of $\left.\left[\mathrm{Ar}_{2} \mathrm{PN}(\mathrm{R}) \mathrm{PAr}_{2}\right)\right] \mathrm{Cr}(\mathrm{CO})_{4}$

合物 $\mathbf{H}$ 与 Wass 等 ${ }^{[36]}$ 报道的原位合成的化合物一致, 但 是通过不同的路径生成.

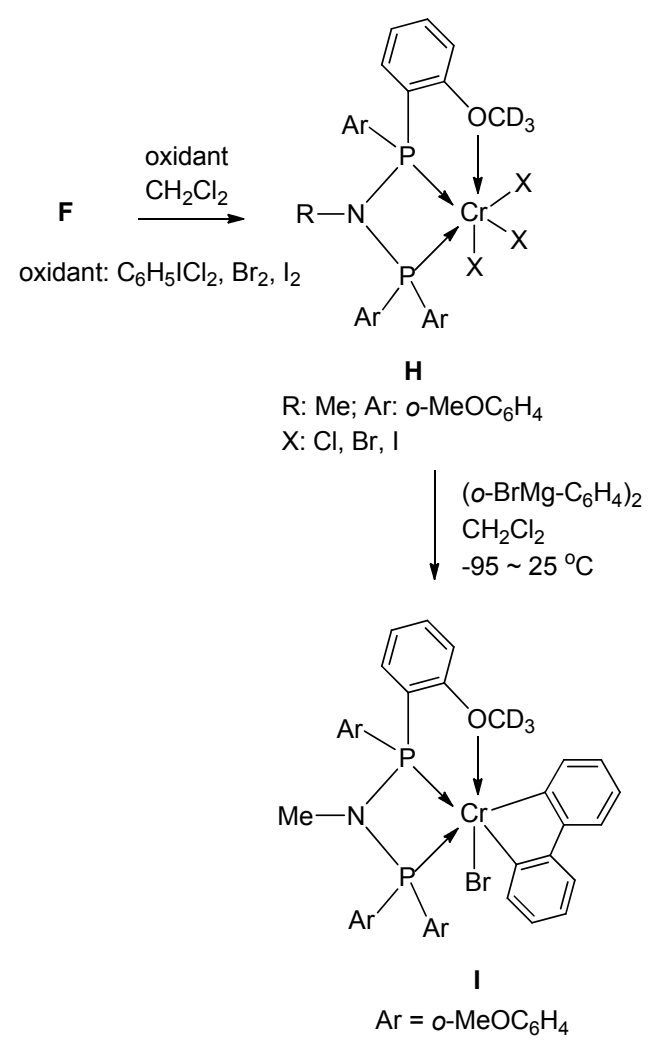

图式 $\left.8\left[\mathrm{Ar}_{2} \mathrm{PN}(\mathrm{R}) \mathrm{PAr}_{2}\right)\right] \mathrm{Cr}(\mathrm{CO})_{4}$ 的氧化反应和官能团转换反 应

Scheme 8 Synthesis and functional group exchange of $\left.\left[\mathrm{Ar}_{2} \mathrm{PN}(\mathrm{R}) \mathrm{PAr}_{2}\right)\right] \mathrm{Cr}(\mathrm{CO})_{4}$

\section{3 助剂以及溶剂}

在烯烃聚合催化中, 我们将能够发生烯烃分子转化 成产物的过渡金属包含的化合物部分称为主催化剂, 辅 助或活化或促进这种转化的主族元素化合物部分称为 助催化剂. 在[PNP]铬基化合物催化的乙烯齐聚反应体 系中，使用的助剂主要有 MAO、改性的甲基铝氧烷 (MMAO 或 MMAO-3A：庚烷溶液, 30\%的甲基被异丁基 取代)、硅胶负载的甲基铝氧烷(MAO-silica)、乙基铝氧 烷(EAO)、三甲基铝(TMA)、三乙基铝(TEA)、异丁基铝 氧烷 $(i-\mathrm{BAO}),\left[\mathrm{H}\left(\mathrm{OEt}_{2}\right)_{2}\right]^{+}\left\{\mathrm{B}\left[3,5-\left(\mathrm{CF}_{3}\right)_{2} \mathrm{C}_{6} \mathrm{H}_{3}\right]_{4}\right\}^{-}, \mathrm{Na}^{+}-$ $\left\{\mathrm{B}\left[3,5\left(\mathrm{CF}_{3}\right)_{2} \mathrm{C}_{6} \mathrm{H}_{3}\right]_{4}\right\}^{-},\left(\mathrm{Me}_{2} \mathrm{PhNH}\right)^{+}\left[\mathrm{B}\left(\mathrm{C}_{6} \mathrm{~F}_{5}\right)_{4}\right]^{-}$, $\mathrm{Ph}_{3} \mathrm{CB}\left(\mathrm{C}_{6} \mathrm{~F}_{5}\right)_{4}, \mathrm{Al}\left(\mathrm{OC}_{6} \mathrm{~F}_{5}\right)_{3},\left[\mathrm{H}\left(\mathrm{OEt}_{2}\right)_{2}\right]^{+}\left[\mathrm{Al}\left(\mathrm{OC}_{6} \mathrm{~F}_{5}\right)_{4}\right]^{-}$, $\left(\mathrm{Ph}_{3} \mathrm{C}\right)^{+}\left[\mathrm{Ta}\left(\mathrm{OC}_{6} \mathrm{~F}_{5}\right)_{6}\right]^{-}, \mathrm{Al}\left(\mathrm{OEt}_{2}\right)\left[\mathrm{OCH}\left(\mathrm{C}_{6} \mathrm{~F}_{5}\right)_{2}\right]_{3}$, $\mathrm{Al}\left(\mathrm{OEt}_{2}\right)\left[\mathrm{OC}\left(\mathrm{CF}_{3}\right)_{3}\right]_{3},\left(\mathrm{Ph}_{3} \mathrm{C}\right)^{+}\left\{\mathrm{Al}\left[\mathrm{OC}\left(\mathrm{CF}_{3}\right)\right]_{4}\right\}^{-},\left(\mathrm{Ph}_{3} \mathrm{C}\right)^{+}$ $\left\{\mathrm{AlF}\left[\mathrm{OC}\left(\mathrm{CF}_{3}\right)_{3}\right]_{3}\right\}^{-},\left(\mathrm{Ph}_{3} \mathrm{C}\right)^{+}\left\{\left[\left(\mathrm{F}_{3} \mathrm{C}\right)_{3} \mathrm{CO}\right]_{3} \mathrm{Al}-\mathrm{F}-\mathrm{Al}-\right.$ $\left.\left[\mathrm{OC}\left(\mathrm{CF}_{3}\right)_{3}\right]_{3}\right\}^{-}$, $\left(\mathrm{Ph}_{3} \mathrm{C}\right)^{+}\left(\mathrm{CB}_{11} \mathrm{H}_{6} \mathrm{Br}_{6}\right)^{-}$. 在这些助剂中, $\mathrm{MAO}$ 最常用. MAO 是通过 TMA 部分水解形成, 结构通 式为 $(\mathrm{MeAlO})_{n}$ 或 $(\mathrm{MeAlOAlMe})_{n}$. 如无特别说明, 商业 用的如 Akzo-Nobel 提供的 MAO 通常含有一定量的游离 的 TMA.

催化活性中心的形成需要主催化剂与助催化剂之 间的作用，但两者之间的作用比较复杂. 迄今为止，这 类催化活性中心的结构仍然报道极少 ${ }^{[37]}$. 相同的铬化 合物前驱体与不同的助催化剂作用产生的催化结果可 能相同也可能相异. 同样, 不同的铬化合物与相同的助 催化剂作用产生的催化结果也会相同或相异. Wass 等 ${ }^{[19]}$ 在 2002 年报道, $\mathrm{Ar}^{o-\mathrm{OMe}}{ }_{2} \mathrm{PN}(\mathrm{Me}) \mathrm{PAr}^{o-\mathrm{OMe}}{ }_{2} / \mathrm{CrCl}_{3}(\mathrm{THF})_{3}$ 主 催化剂和 MAO, MMAO 或硅胶负载的 MAO (MAOsilica)助剂的催化结果基本上一致, 同时 $\mathrm{Ar}^{\mathrm{o}-\mathrm{OMe}}{ }_{2} \mathrm{PN}-$ (Me) $\mathrm{PAr}^{\mathrm{o}-\mathrm{OMe}}{ }_{2} / \mathrm{CrCl}_{3}(\mathrm{THF})_{3} / \mathrm{MAO}$ 与 $\mathrm{Ar}^{\mathrm{o}-\mathrm{OMe}}{ }_{2} \mathrm{PN}(\mathrm{Me})-$ $\mathrm{PAr}^{\mathrm{o}-\mathrm{OMe}}{ }_{2} / \mathrm{CrCl}_{2}$ (tolyl)(THF) $)_{3} / \mathrm{MAO}$ 的结果也相同. 他们 推测有可能形成相同的催化活性中心. Blann 等 ${ }^{[38]}$ 在 2005 年报道, $\mathrm{Ar}^{o-\mathrm{Et}}{ }_{2} \mathrm{PN}(\mathrm{Me}) \mathrm{PAr}^{o-\mathrm{Et}}{ }_{2} / \mathrm{CrCl}_{3}(\mathrm{THF})_{3}$ (或 $\left.\mathrm{Cr}(\mathrm{acac})_{3} 、 \mathrm{Cr}(\mathrm{EH})_{3}\right) / \mathrm{MAO}$ 体系的催化活性和选择性也在 同一个数量级; $\mathrm{Ar}^{\mathrm{o}-\mathrm{Et}}{ }_{2} \mathrm{PN}(\mathrm{Me}) \mathrm{PAr}^{\mathrm{o}-\mathrm{Et}}{ }_{2} / \mathrm{Cr}(\mathrm{acac})_{3} / \mathrm{MAO}$ (或 MMAO-3A、EAO+TMA)的催化结果也相差不大. 上述 Scheme 6 也说明通过与不同助剂反应可以生成相同活 性物种. Gambarotta 和 Duchateau 等 ${ }^{[39]}$ 实验证实 $\left\{\left[\mathrm{Ph}_{2} \mathrm{PN}\right.\right.$ $\left.\left.(\mathrm{Cy}) \mathrm{PPh}_{2}\right] \mathrm{CrCl}_{3}\right\}_{2}$ 与 $\mathrm{TMA}$ 反应则意外地生成蓝色结晶状 固体 $\mathbf{J}$ (Eq. 11).

2007 年大庆石油学院的姜涛课题组研究发现 ${ }^{[40]}$, 对于主催化剂 $\mathrm{Ph}_{2} \mathrm{PN}(i \mathrm{Pr}) \mathrm{PPh}_{2} / \mathrm{Cr}(\mathrm{acac})_{3}, 1$-辛烯的催化 活性和选择性受助剂的影响, MMAO 最好, 其次 MAO, 然后是 EAO 和 $i$ BAO. Gambarotta 和 Duchateau 等 ${ }^{[39]}$ 的 工作已经证明单独使用 TMA 助剂没有催化活性. 因此, 
<smiles>ClC1(Cl)[Ge](Cl)(c2ccccc2)P(Cl)(c2ccccc2)(c2ccccc2)[Te]1(Cl)c1ccccc1</smiles>

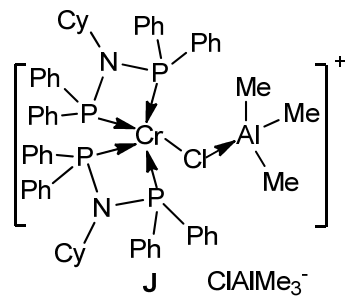

Overett 等 ${ }^{[41]}$ 于同年研究了组合助剂的作用, 即使用烷 基铝作为烷基化试剂，含氟的嗍或铝化合物则作为“拔” 基团试剂. 在组合助剂中, 含氟的硼或铝化合物的用量 较少, 通常 $1 \sim 5$ equiv., 而烷基铝的用量需要 $30 \sim 100$ equiv. 但是, 在使用 $\mathrm{Ph}_{3} \mathrm{CB}\left(\mathrm{C}_{6} \mathrm{~F}_{5}\right)_{4}$ 助剂时, 通过核磁共 振氟谱和磷谱检测发现其与 $[\mathrm{PNP}]$ 配体发生了反应 (Scheme 9), 催化结果给出了非选择性的 Schulz-Flory 分 布的齐聚产物. 随后, McGuinness 和 Tooze 等 ${ }^{[42]}$ 考察了 其它组合助剂的影响. 对于催化体系 $\mathrm{Ph}_{2} \mathrm{PN}(i-\mathrm{Pr}) \mathrm{PPh}_{2} /$ $\mathrm{CrCl}_{3}(\mathrm{THF})_{3} / \mathrm{AlEt}_{3}$ (含其它助剂), 尽管它们显示一定的 活性和齐聚选择性, 但是反应很快会失活. 在反应的过 程中, 组合的助剂之间容易发生基团交换反应，很大程 度上影响了助剂应有的效果.

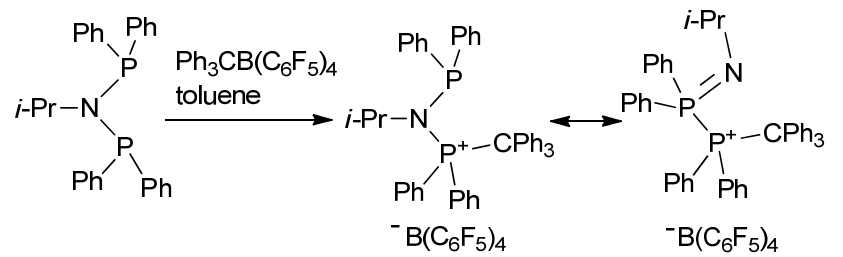

图式 $9 \mathrm{Ph}_{2} \mathrm{PN}(i-\mathrm{Pr}) \mathrm{PPh}_{2}$ 配体与 $\mathrm{Ph}_{3} \mathrm{CB}\left(\mathrm{C}_{6} \mathrm{~F}_{5}\right)_{4}$ 的反应

Scheme 9 Reaction of $\mathrm{Ph}_{2} \mathrm{PN}(i-\mathrm{Pr}) \mathrm{PPh}_{2}$ with $\mathrm{Ph}_{3} \mathrm{CB}\left(\mathrm{C}_{6} \mathrm{~F}_{5}\right)_{4}$

本文所综述的[PNP]配位骨架的铬化合物催化剂体 系如果没有特别说明, 均属于均相反应体系. 同时, 相 比于烯烃聚合催化体系生成的聚合物, 该催化体系得到 的产物主要是 1-辛烯和 1-已烯, 其它如 $\mathrm{C}_{6}$ 环状物、 $\mathrm{C}_{8}$ 以上烯烃和部分 $\alpha$-烯烃的异构体以及聚合物是副产物, 含量不是很高. 因此, 产物也基本上处于溶液状态. 均 相催化反应体系必须要考虑的是溶剂介质. 常用的溶剂 是甲苯和环己烷. Blann 等 ${ }^{[43]}$ 报道乙烯在甲基环己烷中 的溶解度较甲苯的好, 因此会选用甲基环己烷作溶剂. 通常配位型的溶剂如 THF 和 $\mathrm{Et}_{2} \mathrm{O}$ 是禁止使用的, 一方 面这些溶剂可能会影响[PNP]配体与金属铬中心的配位, 另一方面也会影响乙烯分子与铬的配位作用. Bercaw 等 ${ }^{[35]}$ 在使用 $\left[\mathrm{H}\left(\mathrm{OEt}_{2}\right)_{2}\right]^{+}\left\{\mathrm{B}\left[3,5-\left(\mathrm{CF}_{3}\right)_{2} \mathrm{C}_{6} \mathrm{H}_{3}\right]_{4}\right\}^{-}$助剂时会 加入少量的 $\mathrm{Et}_{2} \mathrm{O}$, 可能改善催化剂的溶解性. 同时, 他 也报道在 $\left\{\mathrm{Ph}_{2} \mathrm{PN}\left[\mathrm{CH}_{2}\left(o-\mathrm{CH}_{3} \mathrm{OC}_{6} \mathrm{H}_{4}\right) \mathrm{PPh}_{2}\right] \mathrm{CrCl}_{2}(\mu-\mathrm{Cl})\right\}_{2} /$ $\mathrm{MAO}$ 催化体系中使用氯苯溶剂能够较甲苯有更高的活 性、稳定性和选择性, 但是使用 1,2-二氯代苯的效果不 好. 姜涛等 ${ }^{[44]}$ 研究发现少量卤代试剂如二氯甲烷、三氯
甲烷、四氯甲烷、1,1,2-三氯乙烷、1,1,1-三氯乙烷、1溴丁烷、1-氯丁烷对反应的活性和选择性都会有影 响.

\section{4 催化机理}

\section{1 [PNP]配体铬化合物的电子和立体空间结构}

$[\mathrm{PNP}]$ 配体中氮和磷原子上均有一孤对电子, 总体 上呈 $6 \mathrm{e}$ 结构, 具有很强的亲核性. [PNP]配体的金属化 合物报道不多. 在相应的铬化合物催化剂报道之前，仅 金、银、钼、铇、铑、钯、铂、铼的化合物报道 ${ }^{[45]}$, 主 要用的配体是 $\mathrm{Ph}_{2} \mathrm{PN}(\mathrm{H}) \mathrm{PPh}_{2}$, 其与中心金属的键合方 式主要呈现如下 4 种: (1)螯合配位形成 PNPM 四元环; (2)桥联配位形成 $\mathrm{P}_{4} \mathrm{~N}_{2} \mathrm{M}_{2}$ 八元环或 $\mathrm{P}_{6} \mathrm{~N}_{3} \mathrm{M}_{3}$ 十二元环; (3) 仅 $\mathrm{P}$ 原子端基配位; (4) $\mathrm{N}$ 上质子消除后的端基配位. 磷 原子是软碱电子给体，与过渡金属不但形成 $\mathrm{P} \rightarrow \mathrm{M} \sigma$ 配 位键, 还会形成 $M \rightarrow P \pi$ 反键, 因而配位能力很强. 键合 方式(1)中双磷原子螯合配位可以更好地稳定中心金属， 但是形成的四元环具有强的环张力, 有着反环 “束缚” 的不稳定性. 两者的矛盾往往在磷和氮原子上取代基的 大小和过渡金属的电子特性方面得到妥协.

$[\mathrm{PNP}]$ 配体铬化合物催化剂则是这种妥协下的结构 产物. 一方面, 磷上取代基较氮上取代基对 $\mathrm{Cr}$ 中心的立 体空间影响更大，但是氮上取代基的立体空间伸展性决 定了 $\mathrm{P}-\mathrm{N}-\mathrm{P}$ 的键角. 这种结构对 $\mathrm{Cr}$ 中心的乙烯底物 分子的转换反应性影响极为重要(图 2) ${ }^{[46]}$. 文献报道, 氮原子上线性取代基较面性或体性的能够产生比较大 的 $\mathrm{P}-\mathrm{N}-\mathrm{P}$ 角, 相应的 $\mathrm{P}-\mathrm{Cr}-\mathrm{P}$ 角变小, $\mathrm{Cr}$ 中心官能 基的反应空间变大，这种结构特征我们称为 $\mathrm{N}$ 原子的 $\alpha$ 支化效应 $(\alpha$-branch effect). 另一方面, 磷上的取代基含 有电子给体原子时，易与中心金属配位，且配位的能力 和倾向也远大于氮上取代基的电子给体原子．再一方 面，磷原子和氮原子上取代基的推电子和拉电子性质对 中心金属的稳定配位有着不可忽略的影响. 这可以理解 为这些取代基对 P,N,P-骨架整体的电子结构的影响, 因 而间接地影响到 $[\mathrm{PNP}] \rightarrow \mathrm{Cr}$ 的键合强度. 一些文献研究 发现 ${ }^{[1]}$, 类似的 $[\mathrm{PBP}],[\mathrm{PCP}],[\mathrm{POP}],[\mathrm{PSiP}]$ 配体的铬化

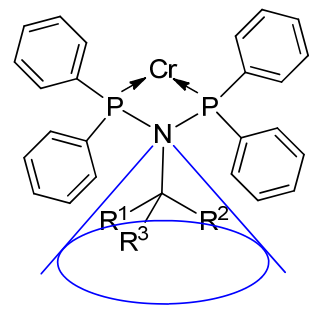

图 2 [PNP]配体氮上取代基的 Tolman 张力角示意图 Figure 2 Tolman expansion angle of the substituents at the $\mathrm{N}$-atom of [PNP]-ligand 
合物没有这方面的催化性能. 显然, 中间氮原子的电子 结构性能对整个[PNP]配体铬催化剂的性能发挥起着重 要的作用. 尽管涉及到 $\beta$-氢消除的热力学和动力学影响 因素, 但是所有这些结构因素对铬中心催化乙烯形成 $\mathrm{CrC}_{6}$ 七元环和 $\mathrm{CrC}_{8}$ 九元环过渡态的稳定性有着质的影 响, 因而也影响到产物 1-已烯和 1-辛烯的选择性.

\section{2 金属环化催化反应机理}

早期的研究表明金属中心作用下的乙烯齐聚产物 往往符合 Schulz-Flory 分布或 Poisson 分布, 是 CosseeArlman 机理作用下的结果, 但是碳链增长速率与碳链 消除速率相差不大 ${ }^{[47]}$. 从基团反应性角度考虑, 金属中 心既容易发生乙烯配位插入反应, 也容易发生 $\beta$-氢迁移 进而还原消除反应. 这一对反应的竞争性体现了金属的 反应能力, 而金属的反应能力又受到周边配体的立体空 间和电子结构的影响.

最初 Ziegler 和 Martin 发现的 “Alphabutol Process” 乙烯二聚生成 1-丁烯被认为经由 $\mathrm{Ti}(\mathrm{II}) / \mathrm{Ti}$ (IV)作用下的 金属环化机理 ${ }^{[48]}$, 但是 $\mathrm{TiC}_{4}$-五元环结构的化合物及其 开环生成 1-丁烯产物的反应未见报道. 结构方面的知识 表明 $\mathrm{TiC}_{4}$-五元环结构比较刚性, 不太容易发生 $\beta$-氢迁 移. 因此, 有人始终认为乙烯二聚生成 1-丁烯应该经由 Cossee-Arlman 反应机理, 只不过链增长生成丁基进而 链转移反应生成 1-丁烯占绝对主导性. 而且使用含有同 位素标记的 $\mathrm{CH}_{2}=\mathrm{CH}_{2} / \mathrm{CD}_{2}=\mathrm{CD}_{2}$ 给出不规则的 $\mathrm{H} / \mathrm{D}$ 齐

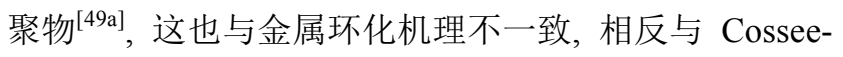
Arlman 机理下的反应产物一致. 但是, Girolami 和他的 合作者们发现双膦配体稳定的或双膦和单茂配体共同 稳定的钛化合物催化剂能够催化乙烯二聚生成 1-丁烯, 而其氢化物或甲基化合物却不能发生乙烯插入反应 ${ }^{[50]}$. 这个结果说明 Cossee-Arlman 的配位插入链增长反应机 理不可行. 那么, 唯一的解释就是有且只能两个烯烃分 子在钛中心环化然后脱除生成 1-丁烯. 脱除的过程是否 涉及逐步的或协同的能量较高的 $\beta$-氢迁移和还原消除 的基元反应仍不清楚.

乙烯高选择性三聚和四聚则可以说明经由金属环 化而非 Cossee-Arlman 反应机理. Manyik 等 ${ }^{[51]}$ 在 1977 年 第一个报道了铬体系催化乙烯选择性生成 1-已烯. 他们 认为该反应机理涉及两个乙烯分子与铬中心作用形成 $\mathrm{CrC}_{4}$ 五元环中间体. 但是, 他们并没有考虑到扩环反 应, 而是提出了环内 $\beta$ 氢一迁移至铬中心导致五元开环 形成 $\mathrm{Cr}(\mathrm{H})\left(\mathrm{CH}_{2} \mathrm{CH}_{2} \mathrm{CH}=\mathrm{CH}_{2}\right)$, 第三个乙烯分子配位插 入到 $\mathrm{Cr}-\mathrm{H}$ 中形成 $\mathrm{Cr}\left(\mathrm{CH}_{2} \mathrm{CH}_{3}\right)\left(\mathrm{CH}_{2} \mathrm{CH}_{2} \mathrm{CH}=\mathrm{CH}_{2}\right)$, 进 而进一步还原消除反应生成 1-已烯, $\mathrm{Cr}$ 活性中心还原继 续引发乙烯三聚. 动力学实验也证明该反应对乙烯是二 级反应, 亦即铬五元环中间体的形成为控速步骤.
McDermott 等 ${ }^{[52]}$ 证明了 $\mathrm{Pt}(\mathrm{II})$ 金属环的存在, 为金属环 化机理提供了依据. $\mathrm{CrC}_{4}$ 五元环中间体也被建议存在于 Phillips 负载型 $\mathrm{CrO}_{3} / \mathrm{SiO}_{2}$ 乙烯聚合催化反应体系中 ${ }^{[53]}$.

随后, Briggs $^{[54]}$ 假设第三个乙烯分子是快速插入 $\mathrm{CrC}_{4}$ 五元环中形成 $\mathrm{CrC}_{6}$ 七元环中间体而非 $\beta$-氢转移乙 烯插入, $\mathrm{CrC}_{6}$ 七元环再发生环内 $\beta$-氢迁移开环形成 $\mathrm{Cr}(\mathrm{H})-\left(\mathrm{CH}_{2} \mathrm{CH}_{2} \mathrm{CH}_{2} \mathrm{CH}_{2} \mathrm{CH}=\mathrm{CH}_{2}\right)$, 进而还原消除出 1己烯，铬活性中心得以再生(Scheme 10). 这一假设得到 McDermott 等 ${ }^{[52]}$ 的热力学实验数据的支持. Briggs 提出, 一方面, 要选择性生成 1-已烯, 这一插入反应必须快速 于 $\mathrm{CrC}_{4}$ 五元环分解为 1-丁烯; 另一方面, 1-己烯的生成 又必须快速于乙烯进一步插入 $\mathrm{CrC}_{6}$ 七元环形成更大的 金属环.

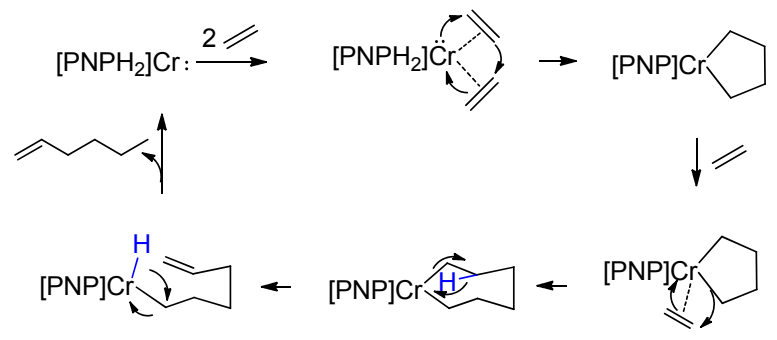

图式 10 乙烯选择性三聚的反应机理

Scheme 10 Mechanism of selective ethylene trimerization

$\mathrm{CrC}_{4}$ 五元环和 $\mathrm{CrC}_{6}$ 七元环化合物的单晶结构均有 报道 ${ }^{[55 \mathrm{a}]}$, 证明了这两类中间体的存在. 相比之下七元 环化合物更容易分解形成 $\alpha$-直链烯烃如 1-已烯 ${ }^{[21]}$. 稳定 的铬烷基氢化物比较少见. Bercaw 等利用 $1: 1$ 的 $\mathrm{C}_{2} \mathrm{D}_{4}$ 和 $\mathrm{C}_{2} \mathrm{H}_{4}$ 进行 $\left[\left((o-\mathrm{MeO}-\mathrm{Phenyl})_{2} \mathrm{P}\right)_{2} \mathrm{NCH}_{3}\right] \mathrm{CrPh}_{3} / \mathrm{H}\left(\mathrm{Et}_{2} \mathrm{O}\right)$ $\mathrm{B}\left(\mathrm{C}_{6} \mathrm{H}_{3}\left(\mathrm{CF}_{3}\right)_{2}\right)_{4}$ 以及 $\left[\left((o-\mathrm{MeO}-\mathrm{Phenyl})_{2} \mathrm{P}\right)_{2} \mathrm{NCH}_{3}\right] \mathrm{CrBr}-$ $\left(o, o^{\prime}\right.$-biphenyldiyl) $/ \mathrm{NaB}\left(\mathrm{C}_{6} \mathrm{H}_{3}\left(\mathrm{CF}_{3}\right)_{2}\right)_{4}$ 催化体系下的三聚 反应, 结果生成 $\mathrm{C}_{6} \mathrm{D}_{12}, \mathrm{C}_{6} \mathrm{D}_{8} \mathrm{H}_{4}, \mathrm{C}_{6} \mathrm{D}_{4} \mathrm{H}_{8}$ 和 $\mathrm{C}_{6} \mathrm{H}_{12}$ 的比率 为 $1: 3: 3: 1$, 含偶数个氛 $\left(\right.$ Scheme 11) ${ }^{[49 b]}$. 这一结果 验证了金属环化机理.

事实上已经报道的研究结果表明铬基催化剂体系 (2-乙基已酸铬/2,5-二甲基吡咯/三乙基铝和二乙基氯化 铝)可以给出近 $95.9 \%$ 的乙烯三聚选择性(其中 1-已烯的 选择性为 $99.5 \%$ ), 而 1 -丁烯的生成量极少或几乎没 有 ${ }^{[55 b]}$. 也就是说 Briggs 提出的 “要选择性生成 1-已烯, 这一插入反应必须快速于 $\mathrm{CrC}_{4}$ 五元环分解为 1-丁烯” 至少对于这类催化剂已经没有必要. 从结构角度考虑, 1-已烯的生成是由于环内 $\beta$-氢迁移开环进而还原消除的 反应. 可以推测 $\mathrm{CrC}_{4}$ 五元环分解为 1-丁烯应该经过类 似的反应过程. 但是这种反应实际上几乎没有发生, 亦 即 $\mathrm{CrC}_{4}$ 五元环环内 $\beta$-氢迁移开环进而还原消除的反应 没有发生. 这说明 $\mathrm{CrC}_{4}$ 五元环的刚性很大程度上阻碍 

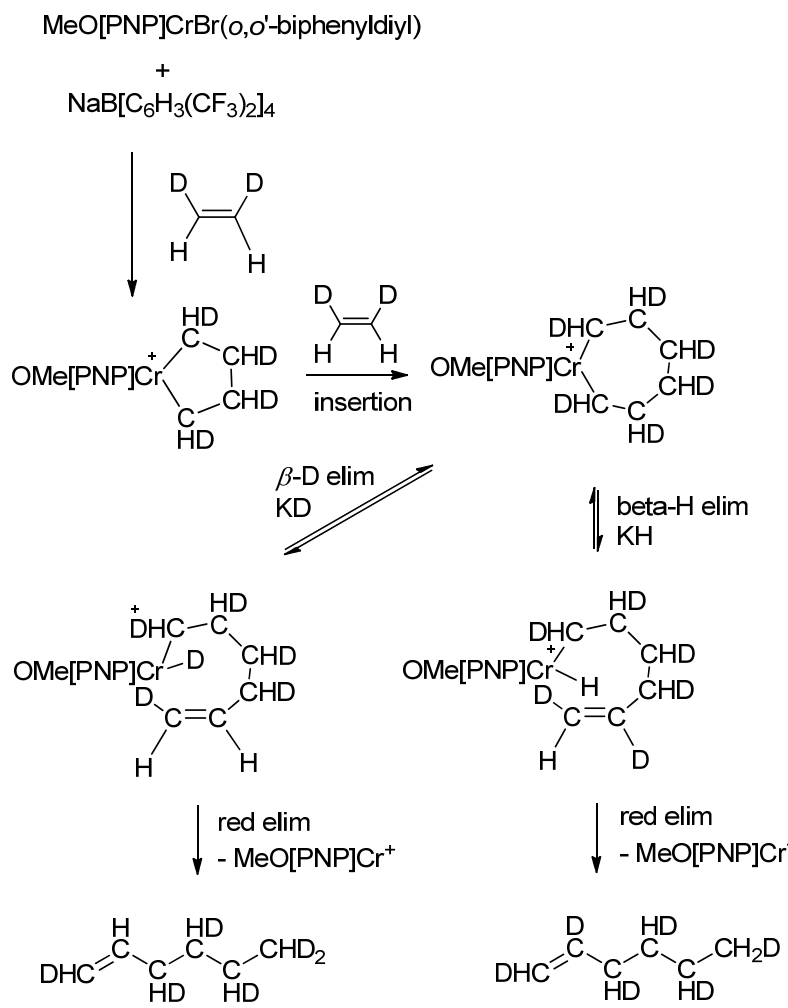

图式 11 以 $1: 1$ 比例乙烯单体 $\mathrm{C}_{2} \mathrm{D}_{4} / \mathrm{C}_{2} \mathrm{H}_{4}$ 的选择性三聚反应 Scheme 11 Selective ethylene trimerization of $1: 1 \mathrm{C}_{2} \mathrm{D}_{4}$ and $\mathrm{C}_{2} \mathrm{H}_{4}$

了反应的发生. 相比较可以发现 $\mathrm{CrC}_{6}$ 七元环的刚性要 远小于 $\mathrm{CrC}_{4}$ 五元环, 结构容易调整, 因而发生了环内 $\beta$ 氢迁移. 1-丁烯的几乎零生成很好地肯定了该铬基催化 剂体系产生 1-已烯的金属环化机理. 这一点 CosseeArlman 机理无法给予解释. 该结论同时也暗示钛基催 化剂高选择性催化乙烯二聚具有独特性, 具体机理尚待 进一步探究.

已有的研究结果也发现 ${ }^{[56]}$, 在选择性三聚反应生 成 $\mathrm{C}_{6}$ 烯烃中除 1-己烯外, 始终含有一定量的甲基环戊 烷和亚甲基环戊烷，比例接近 $1: 1$. 可以推测, $\mathrm{CrC}_{6}$ 七 元环中间体在发生环内 $\beta$-氢迁移后除发生还原消除主 反应外，同时会发生 $\mathrm{Cr}(\mathrm{H})\left(\mathrm{CH}_{2} \mathrm{CH}_{2} \mathrm{CH}_{2} \mathrm{CH}_{2} \mathrm{CH}=\mathrm{CH}_{2}\right)$ 端烯基的配位成环形成 $\mathrm{Cr}(\mathrm{H})\left(\mathrm{CH}_{2}\right.$-cyclo- $\mathrm{CHCH}_{2}$ $\mathrm{CH}_{2} \mathrm{CH}_{2} \mathrm{CH}_{2}$ ), 其进一步还原消除给出甲基环戊烷或再 次 $\beta$-氢迁移给出亚甲基环戊烷，铬活性中心得以复原， 后者还会给出一分子的 $\mathrm{H}_{2}$ (Scheme 12). 甲基环戊烷和 亚甲基环戊烷的比例接近 $1: 1$, 表明这两种副反应的 速率基本上相等. 这两种副产物的生成也是 CosseeArlman 机理无法给予解释的.

Briggs 同时提出了“另一方面, 1-已烯的生成又必须 快速于乙烯进一步插入 $\mathrm{CrC}_{6}$ 七元环形成更大的金属 环”. 但是, Houk 和 $\mathrm{Yu}^{[57 a]}$ 对钽系乙烯齐聚催化体系进

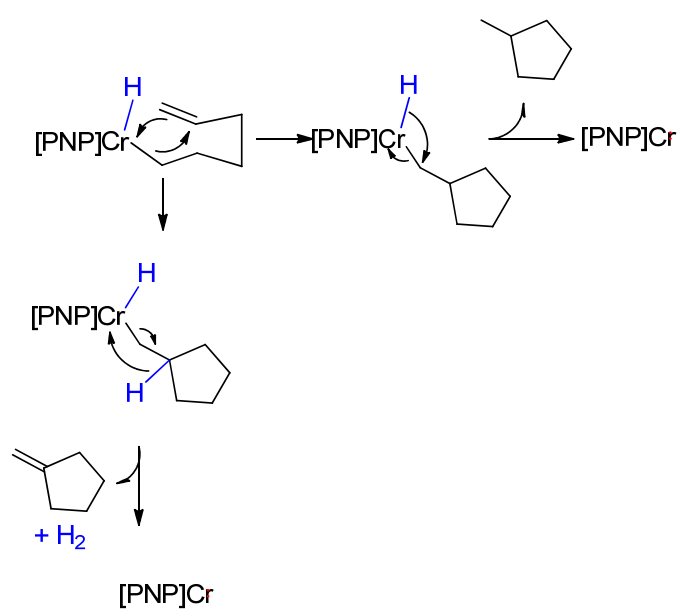

图式 12 乙烯选择性三聚中环状 $\mathrm{C}_{6}$ 的形成机理 Scheme 12 The formation of cyclo- $\mathrm{C}_{6}$ in selective ethylene trimerization

行了理论计算, 认为乙烯分子插入到 $\mathrm{TaC}_{6}$ 环生成四聚 产物的活化能 $(23.1 \mathrm{kcal} / \mathrm{mol})$ 要较插入到 $\mathrm{TaC}_{4}$ 环生成三 聚产物的活化能(13.0 kcal $/ \mathrm{mol})$ 高. Budzelaar 等 ${ }^{[57 b]}$ 对钛 系催化体系的量化计算表明, 形成 $\mathrm{TiC}_{8}$ 环中间体是不许 可的, 其需要的能量要较 $\mathrm{TiC}_{6}$ 环高约 $6 \sim 7 \mathrm{kcal} / \mathrm{mol}$. 不 过, Tobisch 和 Ziegler ${ }^{[58]}$ 计算认为乙烯分子插入到 $\mathrm{TiC}_{4}$ 环形成 $\mathrm{TiC}_{6}$ 环 $(19.5 \mathrm{kcal} / \mathrm{mol})$ 与插入到 $\mathrm{TiC}_{6}$ 环形成 $\mathrm{TiC}_{8}$ 环 $(20.5 \mathrm{kcal} / \mathrm{mol})$ 所需要克服的能垒相当. 因此, 对于钛 系催化体系, 乙烯三聚和四聚均是可能的, 但是受 1-己 烯的脱除速率影响. 如果 1-已烯的脱除速率占主导性, 产物会停留在 1-己烯; 如果 1-已烯的脱除速率与乙烯插 入到七元环或者更大的金属环状物的速率相当或略低, 那么特定的 $\alpha$-烯烃选择性会丧失，代之以统计分布的齐 聚物. 对于 $\mathrm{CrC}_{6}$ 七元环继续插入乙烯分子的理论研究 未有报道. 事实上, 2004 年 Sasol 技术公司的研究人员在 2002 英国石油公司的研究基础上，将磷氮磷配体中磷 原子上芳基取代基由甲氧基变换为氢或烷基基团，成功 实现了乙烯选择性四聚, 但同时包含选择性三聚产物. 也就是说通过对配体的结构调控, 乙烯选择性三聚行为 部分向四聚转化. 这证明了 $\mathrm{CrC}_{8}$ 九元环中间体是可能 形成的. 是否能够形成更大的金属环化物中间体如 $\mathrm{CrC}_{10}$ 十一元环或 $\mathrm{CrC}_{12}$ 十三元环等, 目前这方面的研究 结果很少. 但可以肯定的是环数越大, 稳定性越低, 产 生的可能性也就越小. 乙烯选择性四聚是在三聚基础上 的扩环反应( $\mathrm{Scheme} \mathrm{13),} \mathrm{CrC}_{8}$ 九元环结构类似于 $\mathrm{CrC}_{6}$ 七元环, 容易发生环内 $\beta$-氢迁移. 实验数据发现 $\mathrm{C}_{8}$ 烯烃 的异构体很少, 主要是 1-辛烯. 这表明在四聚过程中仅 发生环内 $\beta$-氢迁移后的还原消除反应. 


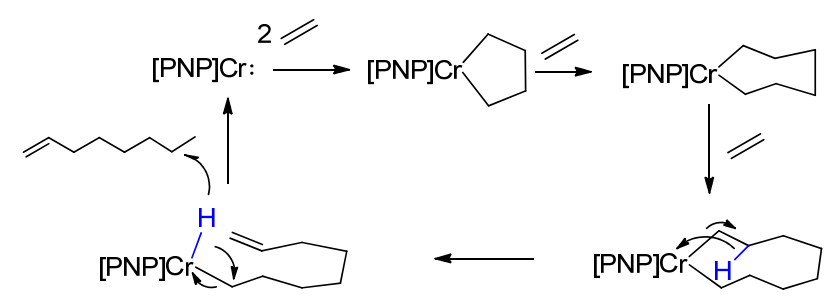

图式 13 乙烯选择性四聚的反应机理

Scheme 13 Mechanism of the selective ethylene tetramerization

在乙烯选择性三聚和/或四聚的催化反应中, 一定 量的高级烯烃或烷烃化合物 $\left(\mathrm{C}_{10^{+}}\right)$被观察到生成. 依据 $\mathrm{C}_{10^{+}}$的结构可以推测 $\mathrm{CrC}_{4}, \mathrm{CrC}_{6}, \mathrm{CrC}_{8}$ 环中间体还会与 生成的产物 1-已烯和/或 1-辛烯发生反应(Scheme 14). 由于中间体的结构因素, 这些反应已不是主要的. 2005 年, Overett 等人针对该方面进行了深入研究 ${ }^{[56]}$.

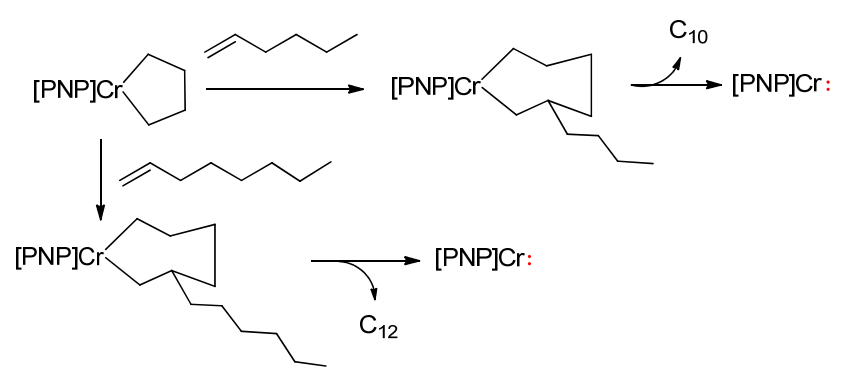

图式 $14 C_{10}$ 和 $C_{12}$ 分子的形成机理

Scheme 14 The formation mechanism of $\mathrm{C}_{10}$ and $\mathrm{C}_{12}$ moleculars

在这些乙烯选择性齐聚反应中, 我们还会观察到聚 合物 PE 或多或少地生成. 从反应机理的角度考虑, 形 成金属环中间体的铬中心应具有两个配位空位. 这样的 活性中心的形成需要经过一系列复杂的基团转化. 例如 对于铬氯化物前驱体, 需要转化为铬烷基或氢化物, 然 后经过消除反应形成所需要的较低氧化态的活性中心. 这个转化过程将有可能涉及到聚合物的生成. 此外, 稳 定的有效的铬配位化合物的形成也至关重要. 单纯的氯 化铬在 MAO 的作用下催化乙烯生成聚合物已有报 道 ${ }^{[59]}$. 很多文献报道了目的配体和铬前驱体可以原位 反应用于催化. 但从工业生产严格控制聚合物的生成来 说，使用高纯度的铬配位化合物应该更符合要求.

在金属环化机理中, 金属氧化态的确定对机理的推 测尤为重要. 2003 年 Morgan 等 ${ }^{[60]}$ 对 Phillips 铬基催化剂 进行了磁性测试, 在二甲苯溶剂中 $\mu_{\mathrm{eff}}$ 值为 $5.17 \mathrm{BM}$, 而 在苯甲醚溶剂中的 $\mu_{\mathrm{eff}}$ 值为 $3.61 \mathrm{BM}$. 前者处于高自旋 态, 对应于 $\mathrm{Cr}(\mathrm{II})$ 化合物; 后者处于较低自旋态, 对应于 $\mathrm{Cr}(\mathrm{III})$ 化合物. 这些结果暗示铬中心处于混合价态. 对 同样的催化体系, Van Rensburg 等 ${ }^{[61]}$ 计算认为是基于 $\mathrm{Cr}(\mathrm{II}) / \mathrm{Cr}(\mathrm{IV})$ 的循环耦合, 而且配体对不同氧化态的铬
中心稳定很重要. 他们通过计算认为 Phillips 三聚催化 体系的吡咯配体具有半不稳定的性质，与金属络合的 $\eta^{5}$ 一模型首选的是 $\mathrm{Cr}(\mathrm{II})$ 中心，有利于金属环伐烷物种的形 成. 而 $\sigma$ 键-模型首选的是 $\mathrm{Cr}(\mathrm{IV})$ 中心，容易促进金属环 状物的产生和构象的变化, 但是无法计算出适合于抓氢 辅助 $\beta$-氢转移释放出 1-已烯的成键模型. 因此, 可能是 这两种模型之间的简单环滑移促进 $\mathrm{Cr}(\mathrm{IV})$ 的金属环庚 烷转化为 $\mathrm{Cr}(\mathrm{II})$ 的已烯物种.

2006 年, Bercaw 等 ${ }^{[34,35]}$ 研究发现 MAO 与 $[\mathrm{PNP}] \mathrm{CrCl}_{3}$ 发生作用，在与乙烯分子的进一步反应产生 类似于 $\{[\mathrm{PNP}] \mathrm{Cr}\}^{+}$的催化活性物种，金属环化和还原消 除可认为是处于 $\mathrm{Cr}^{\mathrm{III}}$ 和 $\mathrm{Cr}^{\mathrm{I}}$ 的氧化态之间的转换反应. 同年, Gambarotta 和 Duchateau 等 ${ }^{[39]}$ 分离了如 Eq. 11 所 示的 $\mathrm{Cr}^{\mathrm{II}}$ 阳离子化合物, 并辅证以磁性 $\left(\mu_{\mathrm{eff}}=4.8 \mu_{\mathrm{B}}\right)$ 分 析. 催化反应则可能是基于 $\mathrm{Cr}^{\mathrm{IV}}$ 和 $\mathrm{Cr}^{\mathrm{II}}$ 的氧化态之间的 转换. 不管如何, 两个乙烯分子是在低价态铬中心作用 下环化加成导致 $\mathrm{M}^{n}$ 变成 $\mathrm{M}^{n+2}$, 再通过还原消除使低价 态铬中心复原 $\left(\mathrm{M}^{n+2}\right.$ 变为 $\left.\mathrm{M}^{n}\right)$.

\section{5 乙烯齐聚方法}

乙烯齐聚反应基本上在可承受一定温度 $(0$ $\left.100{ }^{\circ} \mathrm{C}\right)$ 和压力 $(0.1 \sim 5.0 \mathrm{MPa})$ 的密闭反应釜中进行. 对 于动力学研究, 往往需要一批可连续摚拌的反应釜, 并 最好配备即时取样装置或及时抽样分析装置.

乙烯齐聚反应基本上是一个均相态的反应，反应在 溶液中进行. 一旦催化剂的浓度确定, 反应过程中需要 调控的主要是温度和压力. 齐聚反应产物通过气相色谱 分析. 齐聚催化反应中的相关参数可通过动力学研究确 定. Sasol 技术公司的 Walsh 等 ${ }^{[62]}$ 对乙烯四聚反应进行了 详细的动力学研究. 使用 $\mathrm{Cr}(\mathrm{acac})_{3} /\left(\mathrm{Ph}_{2} \mathrm{P}\right)_{2} \mathrm{~N} i$ - $\mathrm{Pr} / \mathrm{MAO} /$ cumene 催化剂体系, 控制压力为 $4.5 \mathrm{MPa}$, 温度在 $35{ }^{\circ} \mathrm{C}$ 到 $60{ }^{\circ} \mathrm{C}$ 变化, 他们研究发现温度对反应行为的 影响有三个方面. 第一, 催化剂活化的时间随着温度的 升高而缩短, 因此催化剂的活化是温度的函数. 第二, 初始反应速率随着温度的升高而升高. 但是需要注意的 是随着反应温度的升高, 乙烯在溶液中的浓度反而降 低. 第三, 乙烯消耗的趋势随温度的升高而减缓表明催 化剂可能失活. 在控制反应温度为 $45{ }^{\circ} \mathrm{C}$ 、压力在 $3.0 \sim$ $5.0 \mathrm{MPa}$ 范围内每隔 $0.5 \mathrm{MPa}$ 变化时, 可以观察到: 第 一, 催化剂的初始活化时间对每一个压力都差不多, 因 此催化剂的活化不依赖于乙烯的浓度; 第二, 乙烯消耗 趋势的减缓对每一个压力都差不多, 表明相同的一级反 应衰减速率常数; 第三, 乙烯消耗速率随着压力升高而 升高, 但是 $5.0 \mathrm{MPa}$ 压力后的速率变化不大. $5.0 \mathrm{MPa}$ 可 能是反应最佳的临界压力. 
因此, 控制 $45{ }^{\circ} \mathrm{C}$ 反应温度和 $4.5 \mathrm{MPa}$ 压力, 当铬的 量在 $50 \%$ 上下变化时, 反应速率对铬的量呈一级. 这意 味着在溶解性前提下, 铬的用量可以不受限制. 在相同 条件和一定的铬用量下, 乙烯在不同 MAO 用量随时间 的消耗方面的研究表明催化剂的活化在大于或等于 190 当量的 MAO 作用下可以很好地完成. 通常在这种情况 下反应体系绝对 “纯净”, 即指不需要消耗额外 MAO. 在实际反应中, $\mathrm{MAO}$ 量的消耗要大于这个值, 主要是反 应体系会或多或少地含有消耗 MAO 的杂质.

综合上述研究, 催化剂失活仅与温度有关, 而与压 力无关. 反应在 $35 \sim 45{ }^{\circ} \mathrm{C}$ 温度和 $3.0 \sim 4.5 \mathrm{MPa}$ 压力下 最佳.

对产物的组成随温度和压力的变化研究发现, 在 4.5 MPa 下温度升高 $\mathrm{C}_{6}$ 烯烃中的 1-己烯和 $\mathrm{C}_{16+}$ 烯烃的选 择性增加, 而其它如 1-辛烯和 $\mathrm{C}_{10} \sim \mathrm{C}_{14}$ 烯烃的选择性没 有什么影响. $\mathrm{C}_{10} \sim \mathrm{C}_{14}$ 烯烃主要来自次级反应(图 3). 在 $45{ }^{\circ} \mathrm{C}$ 和 4.5 $\mathrm{MPa}$ 压力下, 1-已烯和 1-辛烯的选择性随着 时间逐步降低, 证明了次级反应的发生. 体系压力的降 低会导致 1-已烯的选择性升高和 $\mathrm{C}_{6}$ 环状化合物以及 $\mathrm{C}_{16}$
+的选择性降低. 同时 1-辛烯的选择性也随之降低. 由 此可见降低温度和降低压力对上述产物选择性的影响 行为正好相反. 在同样压力下, 提高温度会引起 1-己烯 和 1-辛烯的共选择性增加, 但也会导致催化剂的失活而 影响催化剂的效率. 温度和压力对 1-已烯和 1-辛烯之间 的选择性的影响实际上与上面阐述的各自的反应机理 一致. 而温度和压力的变化都会导致乙烯在反应溶液中 的浓度变化. 这种变化最终使得 1-辛烯的选择性相对于 1-已烯的选择性有一定的限度. 文献报道的这类催化剂 的 1-辛烯的选择性最多达 75\%, 而 1-己烯的选择性可高 达 93\%. 这两者之间的大小调节还需更深入地研究.

\section{6 催化性能}

2002 年, 英国石油公司(BP)的 Wass 研究小组首次 报道将[PNP]配体引入到铬基催化剂体系 ${ }^{[19]}$ (图 3). 研究 发现, $1 / \mathrm{CrCl}_{3}(\mathrm{THF})_{3} / \mathrm{MAO}$ 在甲苯溶剂中、任意温度(即 不作任何控制)和 $0.1 \mathrm{MPa}$ 乙烯压力下催化反应 $60 \mathrm{~min}$, 得到产物已烯 $\left(\mathrm{C}_{6} \quad 82.2 \%\right)$ 、辛烯 $\left(\mathrm{C}_{8} \quad 1.0 \%\right)$ 和癸烯 $\left(\mathrm{C}_{10}\right.$<smiles>COc1ccccc1P(c1ccccc1OC)N(C)P(c1ccccc1OC)c1ccccc1OC</smiles>

1

$1 / \mathrm{CrCl}_{3}(\mathrm{THF})_{3} / \mathrm{MAO} /$ toluene ambient temp., $0.1 \mathrm{MPa}$ Activity: $8.9 \times 10^{3} \mathrm{~g} /(\mathrm{g}$ Cr.h $)$ Selectivity: (\%) $\mathrm{C}_{6}\left(1-\mathrm{C}_{6}\right): 82.2$ (99.3) $\mathrm{C}_{8}\left(1-\mathrm{C}_{8}\right): 1.0$ (ndr) $\mathrm{C}_{10+}: 14.4$ PE: ndr<smiles>CCc1ccccc1P(c1ccccc1CC)N(C)P(c1ccccc1CC)c1ccccc1CC</smiles>

2

$2 / \mathrm{CrCl}_{3}(\mathrm{THF})_{3} / \mathrm{MAO} /$ toluene amibent temp., $0.1 \mathrm{MPa}$ Activity: $0 \mathrm{~g} /(\mathrm{g} \mathrm{Cr} \cdot \mathrm{h})$ Selectivity: (\%) $\mathrm{C}_{6}\left(1-\mathrm{C}_{6}\right)$ : ndr (ndr) $\mathrm{C}_{8}\left(1-\mathrm{C}_{8}\right): \mathrm{ndr}(\mathrm{ndr})$ $\mathrm{C}_{10+}: \mathrm{ndr}$ PE: ndr

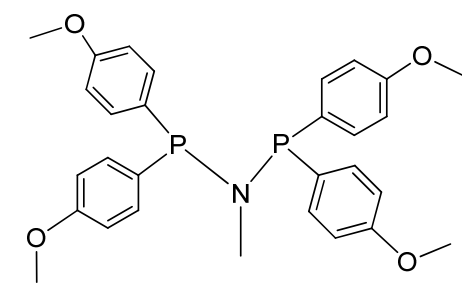

3

$3 / \mathrm{CrCl}_{3}(\mathrm{THF})_{3} / \mathrm{MAO} /$ toluene

ambient temp., $0.1 \mathrm{MPa}$

Activity: $0 \mathrm{~g} /(\mathrm{g} \mathrm{Cr} \cdot \mathrm{h})$

Selectivity: (\%)

$\mathrm{C}_{6}\left(1-\mathrm{C}_{6}\right)$ : ndr (ndr)

$\mathrm{C}_{8}\left(1-\mathrm{C}_{8}\right)$ : ndr (ndr)

$\mathrm{C}_{10+}: \mathrm{ndr}$

PE: ndr<smiles>COc1ccc(OC)c(P(c2cc(OC)ccc2OC)P(c2cc(OC)ccc2OC)N(C)C)c1</smiles>

4

$4 / \mathrm{CrCl}_{3}(\mathrm{THF})_{3} / \mathrm{MAO} /$ toluene ambient temp., $0.1 \mathrm{MPa}$ Activity: $6.7 \times 10^{3} \mathrm{~g} /(\mathrm{g} \mathrm{Cr} \cdot \mathrm{h})$

Selectivity: (\%)

$\mathrm{C}_{6}\left(1-\mathrm{C}_{6}\right): 84.4$ (99.5)

$\mathrm{C}_{8}\left(1-\mathrm{C}_{8}\right): 0.7$ (ndr)

$\mathrm{C}_{10+}: 10.6$

PE: ndr<smiles>COc1ccc(F)cc1N(C)P(c1cc(F)ccc1OC)P(c1cc(F)ccc1OC)c1cc(F)ccc1OC</smiles>

5

$5 / \mathrm{CrCl}_{3}(\mathrm{THF})_{3} / \mathrm{MAO} /$ toluene ambient temp., $0.1 \mathrm{MPa}$ Activity: $6.7 \times 10^{3} \mathrm{~g} /(\mathrm{g} \mathrm{Cr} \cdot \mathrm{h})$ Selectivity: (\%) $\mathrm{C}_{6}\left(1-\mathrm{C}_{6}\right): 83.8(99.4)$ $\mathrm{C}_{8}\left(1-\mathrm{C}_{8}\right): 1.3$ (ndr) $\mathrm{C}_{10+}: 12.5$

PE: ndr

图 $3[\mathrm{PNP}]$ 配体 $\mathbf{1} \sim \mathbf{5}$ 的结构式以及催化结果

Figure 3 Schematic structures of [PNP] ligands $\mathbf{1} \sim \mathbf{5}$ and catalytic results 
$14.4 \%)$, 活性为 $8.90 \times 10^{3} \mathrm{~g} /(\mathrm{g} \mathrm{Cr} \bullet \mathrm{h})$, 其中 $\mathrm{C}_{6}$ 中 $1-\mathrm{C}_{6}$ 的 选择性为 $99.3 \%$. 当上述反应控制 $80{ }^{\circ} \mathrm{C}$ 和不同乙烯压 力时, 产物的种类不变, 比例略有变化 $\left(0.2 \mathrm{MPa}: \mathrm{C}_{6}\right.$ $91.5 \%, \mathrm{C}_{8} 0.6 \%, \mathrm{C}_{10} 7.0 \%$; $0.8 \mathrm{MPa}: \mathrm{C}_{6} 85.0 \%, \mathrm{C}_{8} 0.4 \%$, $\mathrm{C}_{10} 13.4 \%$; $2.0 \mathrm{MPa}: \mathrm{C}_{6} 90.0 \%, \mathrm{C}_{8} 1.8 \%, \mathrm{C}_{10} 8.5 \%$ ), 活性 也逐步提高 $\left[0.2 \mathrm{MPa}: 4.61 \times 10^{3} ; 0.8 \mathrm{MPa}: 9.23 \times 10^{4} ; 2.0\right.$ MPa: $\left.1.03 \times 10^{6} \mathrm{~g} /(\mathrm{g} \mathrm{Cr} \bullet \mathrm{h})\right], \mathrm{C}_{6}$ 中 $1-\mathrm{C}_{6}$ 的选择性稳定在 $99.7 \% \sim 99.9 \%$. 在该温度下, 产物的量与乙烯压力的平 方成正比，这与报道的 2-乙基辛酸铬(III)/2,5-二甲基吡 咯/三乙基铝/卤代烷烃(即 $\mathrm{Cr}(\mathrm{EH})_{3} / \mathrm{DMP} / \mathrm{TEA} /$ Choloralkane)的体系中的催化结果相似 ${ }^{[63]}$.

当反应体系控制温度为 $80{ }^{\circ} \mathrm{C}$, 在保持乙烯压力为 $0.4 \mathrm{MPa}$ 下加入 $1-\mathrm{C}_{4}$ 时, 产物中 $\mathrm{C}_{8}$ 含量明显增加 $\left(\mathrm{C}_{6}\right.$ $60.0 \%, \mathrm{C}_{8} 25.0 \%, \mathrm{C}_{10} 11.0 \%$ ), 活性降低很多 $\left[4.61 \times 10^{4}\right.$ $\mathrm{g} /(\mathrm{g} \mathrm{Cr} \cdot \mathrm{h})]$; 而在任意温度和 $0.1 \mathrm{MPa}$ 乙烯压力下, 当反 应时间延长至 $90 \mathrm{~min}$, 则 $\mathrm{C}_{10}$ 含量明显增加 $\left(\mathrm{C}_{6} 64.1 \%\right.$, $\left.\mathrm{C}_{8} 0.4 \%, \mathrm{C}_{10} 29.0 \%\right)$, 活性为 $8.06 \times 10^{3} \mathrm{~g} /(\mathrm{g} \mathrm{Cr} \bullet \mathrm{h})$, 并出 现产物 $\mathrm{C}_{14}(5.0 \%)$ 和 $\mathrm{C}_{18}(0.6 \%)$. 进一步研究表明, 该催 化体系不催化 $1-\mathrm{C}_{6}$ 三聚生成 $\mathrm{C}_{18}$. 如果在任意温度下保 持乙烯压力为 $0.8 \mathrm{MPa}$ 时, 当加入控制量的 $\mathrm{H}_{2}$ 时, 催化 活性提高 ${ }^{[64]}$, 为 $1.75 \times 10^{5} \mathrm{~g} /(\mathrm{g} \mathrm{Cr} \bullet \mathrm{h})$, 产物的种类和选 择性变化不大 $\left(\mathrm{C}_{6} 81.3 \%, \mathrm{C}_{8} 0.4 \%, \mathrm{C}_{10} 15.4 \%\right)$, 产物中明 确没有已烷.

这些结果表明, 产物不具有 Schulz-Flory 或 Poisson 分布特征, 不受 $\mathrm{H}_{2}$ 导入的影响, 该催化体系遵循金属环 化反应机理而不是 Cossee-Arlman 机理. 加入 $1-\mathrm{C}_{4}$ 引起 产物中 $\mathrm{C}_{8}$ 含量的明显增加可以解释为 1- $\mathrm{C}_{4}$ 插入到 $\mathrm{CrC}_{4}$ 环中的反应结果, $\mathrm{C}_{10}$ 含量的变化可以认为是生成的 1- $\mathrm{C}_{6}$ 插入到 $\mathrm{CrC}_{4}$ 环中的结果, 而产物 $\mathrm{C}_{14}$ 的生成则可能 是 1- $\mathrm{C}_{8}$ 插入到 $\mathrm{CrC}_{6}$ 环中或 1- $\mathrm{C}_{6}$ 插入到 $\mathrm{CrC}_{8}$ 环中. 1- $\mathrm{C}_{6}$ 不能三聚生成 $\mathrm{C}_{18}$, 但产物 $\mathrm{C}_{18}$ 的生成推测则是复杂的环 插入反应作用下的结果. 但是, $\mathrm{H}_{2}$ 的导入对催化活性的 提高的原因不甚明晰 ${ }^{[64]}$.

Wass 等 ${ }^{[20]}$ 还考察了不同铬源以及助剂作用下的催 化性能. 对于 $\mathbf{1} / \mathrm{CrCl}_{3}(\mathrm{THF})_{3} / \mathrm{MAO}$ 体系, 当使用 $\mathrm{CrCl}_{2}-$ (tolyl)(THF) 3 替代 $\mathrm{CrCl}_{3}(\mathrm{THF})_{3}$, 催化结果与相同条件下 的类似，但是这种替代会改善催化剂的溶解性. 同样可 以发现, $\mathrm{CrCl}_{2}$ 也是一个活性铬源. 因此, 不同氧化态的 铬源前驱体在 MAO 的活化下应该生成类似或相同的活 性组分. 当使用修饰过的 MAO(即 MMAO, 部分甲基为 丁基取代)时, 催化结果也基本上相同. 推广到硅胶负 载的 MAO, 催化活性与选择性并没有降低, 同时不生 成聚乙烯副产物. 这点在工艺技术方面非常重要. 如果 有过多的聚合物生成, 往往会黏釜, 引发堵塞现象.

对于 [PNP]配体, 骨架原子上的取代基对铬中心的
电子和空间结构也有很大的影响. 如果将 $\mathbf{1}$ 中磷原子上 芳基的邻位甲氧基变换为乙基(即 2), 结果是没有活性; 将此甲氧基换到对位(即 3), 也没有活性; 保持邻位甲 氧基不变, 在间位引入甲氧基或氟原子(即配体 $\mathbf{4}$ 和 $\mathbf{5}$ ), 结果有活性. 在任意温度和 $0.1 \mathrm{MPa}$ 乙烯压力下, 活性 与选择性与上述相同条件下的反应结果相似 $[6.65 \times$ $10^{3} \sim 7.74 \times 10^{3} \mathrm{~g} /(\mathrm{g} \mathrm{Cr} \bullet \mathrm{h}) ; \mathrm{C}_{6} 83.8 \% \sim 84.4 \%, \mathrm{C}_{8} 0.7 \% \sim$ $1.3 \%, \mathrm{C}_{10} 10.6 \% \sim 12.5 \%$ ]. 显然, 邻位甲氧基的存在非 常重要, 推测该基团可能参与了金属中心的配位，对催 化活性起一定的促进作用.

2004 年, 以南非 Sasol 技术公司为首的研究团队 Bollmann 等在 Wass 等 ${ }^{[20]}$ 的工作基础上研究发现, 对 [PNP] 配体中的取代基进行替换(图 4), 可以调变 $\mathrm{C}_{6}$ 和 $\mathrm{C}_{8}$ 的选择性. 无论从工业应用角度还是学术方面, 该研 究都具有突破性. 一方面, 乙烯三聚制备 1- $\mathrm{C}_{6}$ 的 Philips 催化剂 ${ }^{[55 b]}$ 已经开发, 但是乙烯四聚催化几乎没有报道. 另一方面, 目前研究表明乙烯-辛烯共聚物(POE)相对于 其它聚烯烃具有更好的力学和弹性. $1-\mathrm{C}_{8}$ 将成为有潜力 的共聚单体.

催化体系 $6 \sim 10 / \mathrm{CrCl}_{3}(\mathrm{THF})_{3} / \mathrm{MAO}$ 在甲苯溶剂中 $65{ }^{\circ} \mathrm{C}$ 和 $3.0 \mathrm{MPa}$ 乙烯压力下反应 $30 \mathrm{~min}$, 得到 $\mathrm{C}_{6}$ $\left(24.8 \% \sim 32.7 \%, 1-\mathrm{C}_{6}\right.$ 的选择性为 $39.4 \% \sim 86.5 \%$, 主要 副产物为甲基环戊烷和亚甲基环戊烷)、 $\mathrm{C}_{8}(58.1 \%$ $61.6 \%, 1-\mathrm{C}_{8}$ 的选择性为 $\left.94.1 \% \sim 99.3 \%\right) 、 \mathrm{C}_{10^{+}}(6.4 \% \sim$ $11.7 \%)$ 和 PE $(0.3 \% \sim 1.4 \%)$, 活性为 $8.05 \times 10^{3} \sim 4.36 \times$ $10^{4} \mathrm{~g} /(\mathrm{g} \mathrm{Cr} \cdot \mathrm{h})$, 乙烯四聚的选择性均在 $60 \%$ 左右. 从结 构上分析, $\mathrm{N}$ 原子上的取代基对乙烯四聚的选择性影响 不大, 但会大大地影响活性和三聚过程中 $1-\mathrm{C}_{6}$ 的选择 性. 其中获得 1- $\mathrm{C}_{6}$ 高选择性的是异丙基和环己基, 表明 $\mathrm{N}$ 原子上的取代基 $\alpha$-分支效应的影响.

相比于 $9 / \mathrm{CrCl}_{3}(\mathrm{THF})_{3} / \mathrm{MAO}$ 在 $65{ }^{\circ} \mathrm{C} 、 3.0 \mathrm{MPa}$ 条 件下的催化结果 [活性 $1.17 \times 10^{4} \mathrm{~g} /(\mathrm{g} \mathrm{Cr} \bullet \mathrm{h}) ; \mathrm{C}_{6} 32.7 \%$ (1- $\left.\mathrm{C}_{6} 86.5 \%\right), \mathrm{C}_{8} 60.6 \%\left(1-\mathrm{C}_{8} 99.2 \%\right), \mathrm{C}_{10}+6.4 \%$, $\mathrm{PE}$ $0.3 \%$ ], 催化体系 $9 / \mathrm{Cr}(\mathrm{acac})_{3} / \mathrm{MAO}$ 在 $45{ }^{\circ} \mathrm{C}$ 和 $4.5 \mathrm{MPa}$ 乙烯压力下反应 $30 \mathrm{~min}$ 的活性和乙烯四聚选择性均有 提高[活性 $2.72 \times 10^{5} \mathrm{~g} /(\mathrm{g} \mathrm{Cr} \bullet \mathrm{h}) ; \mathrm{C}_{6} 16.9 \%\left(1-\mathrm{C}_{6} 70.3 \%\right)$, $\mathrm{C}_{8} 68.3 \%$ (1- $\left.\mathrm{C}_{8} 98.8 \%\right), \mathrm{C}_{10+}$ 13.7\%, PE 1.05\%]. 提高的 原因是反应温度的降低和乙烯压力的提高. 这种调节既 增加了乙烯的溶解度也在一定程度上增加了催化剂的 稳定性. 当该反应体系中加入 $0.25 \mathrm{MPa}$ 氢气时, $\mathrm{PE}$ 的量 降低, 而催化活性和选择性变化不大[活性 $2.85 \times 10^{5}$ $\mathrm{g} /(\mathrm{g} \quad \mathrm{Cr} \bullet \mathrm{h}) ; \mathrm{C}_{6} \quad 16.6 \%\left(1-\mathrm{C}_{6} 65.6 \%\right), \mathrm{C}_{8} \quad 68.9 \% \quad\left(1-\mathrm{C}_{8}\right.$ 98.5\%), $\mathrm{C}_{10+}$ 14.5\%, PE 0.07\%].

$\mathrm{P}$ 原子上取代基的变化也对乙烯四聚的选择性有一 定影响. 使用大体积芳香取代基的配体，即 11 12/ 


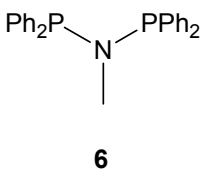

$6 / \mathrm{CrCl}_{3}(\mathrm{THF})_{3} / \mathrm{MAO} /$ toluene $65^{\circ} \mathrm{C}, 3.0 \mathrm{MPa}$

Activity: $2.7 \times 10^{4} \mathrm{~g} /(\mathrm{g} \mathrm{Cr} \cdot \mathrm{h})$ Selectivity: (\%)

$\mathrm{C}_{6}\left(1-\mathrm{C}_{6}\right): 24.8$ (39.4)

$\mathrm{C}_{8}\left(1-\mathrm{C}_{8}\right): 59.0$ (94.1)

$\mathrm{C}_{10+}: \mathrm{ndr}$

PE: 1.4

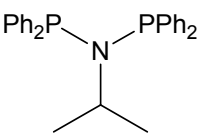

9/Cr(acac) $3 / \mathrm{MAO} /$ toluene $45^{\circ} \mathrm{C}, 4.5 \mathrm{MPa}$

Activity: $2.7 \times 10^{5} \mathrm{~g} /(\mathrm{g} \mathrm{Cr} \cdot \mathrm{h})$

Selectivity: (\%)

$\mathrm{C}_{6}\left(1-\mathrm{C}_{6}\right): 16.9(70.3)$

$\mathrm{C}_{8}\left(1-\mathrm{C}_{8}\right): 68.3(98.8)$

$\mathrm{C}_{10+}: \mathrm{ndr}$

PE: 1.1

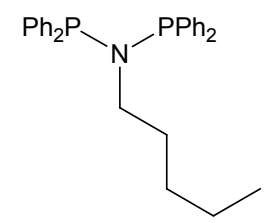

7

$7 / \mathrm{CrCl}_{3}(\mathrm{THF})_{3} / \mathrm{MAO} /$ toluene $65^{\circ} \mathrm{C}, 3.0 \mathrm{MPa}$

Activity: $4.4 \times 10^{4} \mathrm{~g} /(\mathrm{g} \mathrm{Cr} \cdot \mathrm{h})$ Selectivity: (\%)

$\mathrm{C}_{6}\left(1-\mathrm{C}_{6}\right): 24.9$ (54.7)

$\mathrm{C}_{8}\left(1-\mathrm{C}_{8}\right): 58.1(96.8)$

$\mathrm{C}_{10+}: \mathrm{ndr}$

PE: 0.3<smiles>Pc1ccccc1</smiles>

$8 / \mathrm{CrCl}_{3}(\mathrm{THF})_{3} / \mathrm{MAO} /$ toluene $65^{\circ} \mathrm{C}, 3.0 \mathrm{MPa}$

Activity: $8.1 \times 10^{3} \mathrm{~g} /(\mathrm{g} \mathrm{Cr} \cdot \mathrm{h})$ Selectivity: (\%)

$\mathrm{C}_{6}\left(1-\mathrm{C}_{6}\right): 32.1$ (86.1)

$\mathrm{C}_{8}\left(1-\mathrm{C}_{8}\right): 59.4$ (99.3)

$\mathrm{C}_{10+}$ : ndr

PE: 0.5

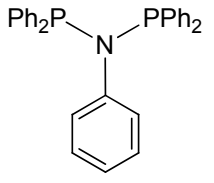

10

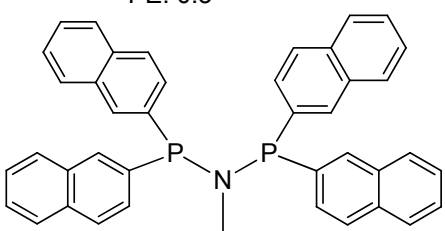

11

$10 / \mathrm{CrCl}_{3}(\mathrm{THF})_{3} / \mathrm{MAO} /$ toluene $65^{\circ} \mathrm{C}, 3.0 \mathrm{MPa}$

$11 / \mathrm{CrCl}_{3}(\mathrm{THF})_{3} / \mathrm{MAO} /$ toluene $65^{\circ} \mathrm{C}, 3.0 \mathrm{MPa}$

Activity: $5.3 \times 10^{4} \mathrm{~g} /(\mathrm{g} \mathrm{Cr} \cdot \mathrm{h})$

Selectivity: (\%)

$\mathrm{C}_{6}\left(1-\mathrm{C}_{6}\right): 26.0(38.7)$

$\mathrm{C}_{8}\left(1-\mathrm{C}_{8}\right): 54.2(93.4)$

$\mathrm{C}_{10+}: \mathrm{ndr}$

PE: 1.3

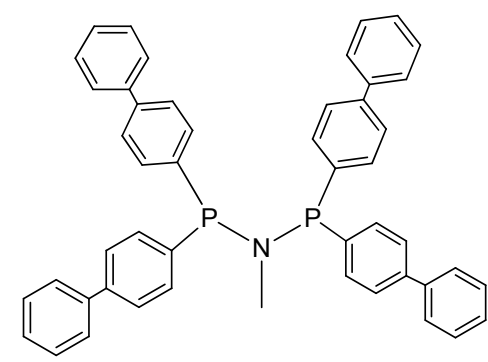

12

$12 / \mathrm{Cr}(\mathrm{acac})_{3} / \mathrm{MAO} /$ toluene $45^{\circ} \mathrm{C}, 4.5 \mathrm{MPa}$

Activity: $3.1 \times 10^{4} \mathrm{~g} /(\mathrm{g} \mathrm{Cr} \cdot \mathrm{h})$

Selectivity: (\%)

C6 (1-C6): 22.9 (38.6)

C8 (1-C8): 56.1 (95.3)

$\mathrm{C}_{10+}: \mathrm{ndr}$

PE: 1.8

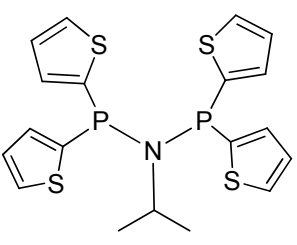<smiles>CCN(C)CC</smiles>

$\begin{array}{ll}\text { 13/Cr(acac) })_{3} / \mathrm{MAO} / \text { toluene } & 14 / \mathrm{Cr}(\mathrm{acac})_{3} / \mathrm{MAO} / \text { toluene } \\ 45^{\circ} \mathrm{C}, 4.5 \mathrm{MPa} & 45^{\circ} \mathrm{C}, 4.5 \mathrm{MPa} \\ \text { Activity: } 1.7 \times 10^{4} \mathrm{~g} /(\mathrm{g} \mathrm{Cr} \cdot \mathrm{h}) & \text { Activity: } 4.4 \times 10^{3} \mathrm{~g} /(\mathrm{g} \mathrm{Cr} \cdot \mathrm{h}) \\ \text { Selectivity: (\%) } & \text { Selectivity: }(\%) \\ \text { C6 (1-C6): } 16.6(42.5) & \mathrm{C} 6(1-\mathrm{C} 6): 16.8(64.6) \\ \mathrm{C} 8 \text { (1-C8): } 60.3(96.6) & \mathrm{C} 8(1-\mathrm{C} 8): 45.2(97.4) \\ \mathrm{C}_{10+} \text { : ndr } & \mathrm{C}_{10+} \text { : ndr } \\ \text { PE: } 2.4 & \text { PE: } 13.6\end{array}$

图 4 [PNP]配体 6 14 的结构式以及催化结果

Figure 4 Schematic structures of [PNP] ligands $6 \sim 14$ and catalytic results

$\mathrm{CrCl}_{3}(\mathrm{THF})_{3} / \mathrm{MAO}$ 体系在 $65{ }^{\circ} \mathrm{C} 、 3.0 \mathrm{MPa}$ 条件下, 乙烯 四聚的选择性会有降低 $(54 \% \sim 56 \%)$. 而在 $45{ }^{\circ} \mathrm{C} 、 4.5$ $\mathrm{MPa}$ 条件下，含噻吩取代基的配体催化剂体系 $13 / \mathrm{CrCl}_{3}(\mathrm{THF})_{3} / \mathrm{MAO}$ 的乙烯四聚选择性达到 $60 \%$, 但 是乙基取代的配体催化剂体系 $14 / \mathrm{CrCl}_{3}(\mathrm{THF})_{3} / \mathrm{MAO}$ 在 相同条件下的四聚选择性仅为 $45 \%$.

将[PNP]配位骨架调变到[PNNP]和[PCCP]骨架[后
者如二苯基膦乙烷(DPPE)], 其与 $\mathrm{CrCl}_{3}(\mathrm{THF})_{3} / \mathrm{MAO}$ 组 成的体系也具有四聚催化活性[前者: 活性 $2.62 \times 10^{4}$ $\mathrm{g} /(\mathrm{g} \quad \mathrm{Cr} \bullet \mathrm{h}) ; \mathrm{C}_{6} 25.2 \%\left(1-\mathrm{C}_{6} \quad 69.6 \%\right), \mathrm{C}_{8} \quad 58.8 \%\left(1-\mathrm{C}_{8}\right.$ $98.4 \%), \mathrm{C}_{10}+8.0 \%$, PE $8.0 \%$; 后者: 活性 $2.48 \times 10^{4} \mathrm{~g} /(\mathrm{g}$ $\mathrm{Cr} \bullet \mathrm{h}) ; \mathrm{C}_{6} 19.7 \%\left(1-\mathrm{C}_{6} 38.2 \%\right), \mathrm{C}_{8} 39.2 \%\left(1-\mathrm{C}_{8} 96.6 \%\right)$, $\mathrm{C}_{10+}$ 6.0\%, PE 35.1\%].

使用其它活化助剂, 如 9/Cr(acac) ${ }_{3} / \mathrm{MMAO}-3 \mathrm{~A}$ 
(EAO/TMA 或 $\left.\mathrm{MAO} / \mathrm{SiO}_{2} / \mathrm{TMA}\right)$, 都能高活性和高选择 性催化乙烯四聚 $\left[2.74 \times 10^{4} \sim 2.85 \times 10^{5} \mathrm{~g} /(\mathrm{g} \mathrm{Cr} \bullet \mathrm{h}), \mathrm{C}_{8}\right.$ 68.6\% 71.6\%]. 使用环己烷代替甲苯溶剂, 催化体系 $9 / \mathrm{Cr}(\mathrm{acac})_{3} / \mathrm{MAO}$ 的反应活性可以提高到 $5.91 \times 10^{5} \mathrm{~g} /(\mathrm{g}$ $\mathrm{Cr} \cdot \mathrm{h})$, 四聚选择性基本上保持不变.

以上的催化剂体系均是原位混合用于反应. 如使用 铬的配合物 $\left[\mathrm{Cr}(\mathbf{1 0}) \mathrm{Cl}_{2}(\mu-\mathrm{Cl})\right]_{2}$ 与 $\mathrm{MAO}$ 组成的体系进行 反应, 也能给出良好的四聚选择性和活性 $\left(8.80 \times 10^{3}\right.$ $\left.\mathrm{g} /(\mathrm{g} \mathrm{Cr} \bullet \mathrm{h}), \mathrm{C}_{8} 61.6 \%\right)$.

2005 年, Sasol 技术公司的 Overett 等 ${ }^{[65]}$ 针对 P 原子 上芳环取代基的电子性质和位置对乙烯齐聚催化活性 的影响(图 5), 研究了通过改变磷原子上芳环取代基的 极性和位置来有效调控乙烯选择性齐聚, 也即对前两者 的工作进行更为具体的探究.

随着甲氧取代基的位置从邻位(即 1)变换到间位(即 18), 再到对位(即 3), 其与 $\mathrm{Cr}(\mathrm{acac})_{3} / \mathrm{MAO}$ 组成的催化剂 的选择性也随之变化, 产物从三聚为主, 向四聚转化.
更接近于 $4 / \mathrm{Cr}(\mathrm{acac})_{3} / \mathrm{MAO}$ 的选择性. 18/ $\mathrm{Cr}(\mathrm{acac})_{3} / \mathrm{MAO}$ 的齐聚活性较低, 给出较多的 PE, 但其三聚和四聚的选 择性还是遵循这种变化趋势.

比较配体 3 和 $19, \mathrm{~N}$ 上的 $\mathrm{R}$ 基团由甲基变为异丙基 时，催化剂的活性及三聚和四聚的选择性都会有所提 高. 采用 $19 / \mathrm{Cr}(\mathrm{acac})_{3} / \mathrm{MAO}$ 可以获得最好的四聚结果, 活性达到 $1.13 \times 10^{5} \mathrm{~g} /(\mathrm{g} \mathrm{Cr} \bullet \mathrm{h}), \mathrm{C}_{8}$ 的选择性达到 $68 \%$. 比 较 $9 / \mathrm{Cr}(\mathrm{acac})_{3} / \mathrm{MAO}$ 的结果, 虽然活性有所下降, 但能 够获得相近的 $\mathrm{C}_{8}$ 及更高的 $\mathrm{C}_{6}$ 选择性. 其它一些产物包 括环状化合物，如甲基环戊烷和亚甲基环戊烷，还有与 1-已烯和 1-辛烯共聚得到的 $\mathrm{C}_{10}$ 到 $\mathrm{C}_{14}$ 齐聚产物。

一般来说, $45{ }^{\circ} \mathrm{C}, 4.5 \mathrm{MPa}$ 条件所获得的活性要比 $65{ }^{\circ} \mathrm{C} 、 3.0 \mathrm{MPa}$ 条件下的活性高. 这主要是由于高乙烯 压力能增大乙烯在反应溶液中的浓度, 较低的温度则降 低催化剂的失活速率。同时，针对 $3(6,9,15,18$, 19) $/ \mathrm{Cr}$ (acac) $)_{3} / \mathrm{MAO}$ 体系, 他们发现 PE 在 $45{ }^{\circ} \mathrm{C} 、 4.5 \mathrm{MPa}$ 条件下的生成量(对应于 $12 \%, 40 \%, 1 \%, 7 \%, 19 \%, 3 \%$ )总 $3 / \mathrm{Cr}(\mathrm{acac})_{3} / \mathrm{MAO}$ 的四聚催化选择性较 $1 / \mathrm{Cr}(\mathrm{acac})_{3} / \mathrm{MAO}$

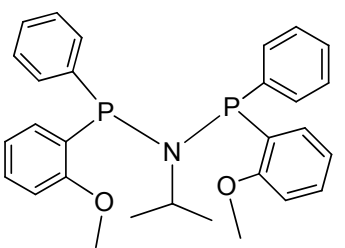

15

$15 / \mathrm{Cr}(\mathrm{acac})_{3} / \mathrm{MAO} /$ toluene $45^{\circ} \mathrm{C}, 4.5 \mathrm{MPa}$

Activity: $4.6 \times 10^{4} \mathrm{~g} /(\mathrm{g} \mathrm{Cr} \cdot \mathrm{h})$

Selectivity: (\%)

$\mathrm{C}_{6}\left(1-\mathrm{C}_{6}\right): 48(>99)$

$\mathrm{C}_{8}\left(1-\mathrm{C}_{8}\right): 6$ (>99)

$\mathrm{C}_{10+}: 33$

PE: 7

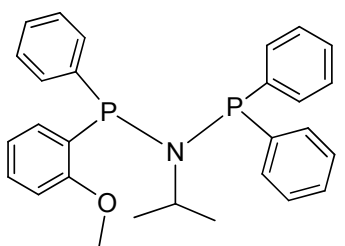

16

$16 / \mathrm{Cr}(\mathrm{acac})_{3} / \mathrm{MAO} /$ toluene $45^{\circ} \mathrm{C}, 4.5 \mathrm{MPa}$ Activity: $2.4 \times 10^{5} \mathrm{~g} /(\mathrm{g} \mathrm{Cr} \cdot \mathrm{h})$ Selectivity: (\%) $\mathrm{C}_{6}\left(1-\mathrm{C}_{6}\right): 63$ (98) $\mathrm{C}_{8}\left(1-\mathrm{C}_{8}\right): 17(98)$ $\mathrm{C}_{10+}: 19$ $\mathrm{PE}:<1$

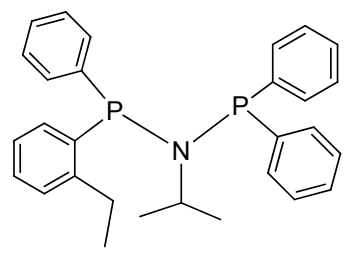

17

$17 / \mathrm{Cr}$ (acac) $)_{3} / \mathrm{MAO} /$ toluene $45^{\circ} \mathrm{C}, 4.5 \mathrm{MPa}$ Activity: $1.6 \times 10^{5} \mathrm{~g} /(\mathrm{g} \mathrm{Cr} \cdot \mathrm{h})$ Selectivity: (\%) $\mathrm{C}_{6}\left(1-\mathrm{C}_{6}\right): 27(72)$ $\mathrm{C}_{8}\left(1-\mathrm{C}_{8}\right): 63(98)$ $\mathrm{C}_{10+}: 5$ PE: $<1$<smiles>COc1cccc(P(c2cccc(OC)c2)N(C)P(c2cccc(OC)c2)c2cccc(OC)c2)c1</smiles>

18

$18 / \mathrm{Cr}(\mathrm{acac})_{3} / \mathrm{MAO} /$ toluene $45^{\circ} \mathrm{C}, 4.5 \mathrm{MPa}$ Activity: $2.5 \times 10^{4} \mathrm{~g} /(\mathrm{g} \mathrm{Cr} \cdot \mathrm{h})$ Selectivity: (\%) $\mathrm{C}_{6}\left(1-\mathrm{C}_{6}\right): 16(55)$ $\mathrm{C}_{8}\left(1-\mathrm{C}_{8}\right): 22(90)$ $\mathrm{C}_{10+}: 10$ PE: 19<smiles>COc1ccc(P(c2ccc(OC)cc2)N(C(C)C)P(c2ccc(OC)cc2)c2ccc(OC)cc2)cc1</smiles>

19

$19 / \mathrm{Cr}(\mathrm{acac})_{3} / \mathrm{MAO} /$ toluene $45^{\circ} \mathrm{C}, 4.5 \mathrm{MPa}$ Activity: $1.1 \times 10^{5} \mathrm{~g} /(\mathrm{g} \mathrm{Cr} \cdot \mathrm{h})$ Selectivity: (\%) $\mathrm{C}_{6}\left(1-\mathrm{C}_{6}\right): 24(74)$ $\mathrm{C}_{8}\left(1-\mathrm{C}_{8}\right): 68$ (99) $\mathrm{C}_{10+}: 4$

PE: 3

图 5 [PNP]配体 15 19 的结构式以及催化结果

Figure 5 Schematic structures of [PNP] ligands $15 \sim 19$ and catalytic results 
体上要比 $65{ }^{\circ} \mathrm{C} 、 3.0 \mathrm{MPa}$ 条件下的量(对应于 $<1 \%, 1 \%$, $<1 \%, 1 \%, 11 \%, 1 \%)$ 多, 并且也会观察到 $\mathrm{C}_{6}$ 向 $\mathrm{C}_{8}$ 类化合 物的部分转化.

对于[PNP]配体 $\mathrm{P}$ 原子上芳基的邻位取代, 取代基 个数的减少, 如从 4 到 0 , 相应的催化选择性会从三聚 为主转化为四聚为主 ${ }^{[37]}$. 这说明该催化体系的选择性 可以通过取代基效应加以调控. 为了确定这种邻位甲氧 基取代对催化体系选择性的影响是基于取代基的空间 位阻还是由其配位性决定, 他们制备了含有两个邻位甲 氧基的配体 15 和只含一个邻位甲氧基的配体 16, 并考 察了其催化性能. 有趣的是, 邻位甲氧基个数的减少对 选择性的影响比较小. 对于 $16 / \mathrm{Cr}(\mathrm{acac})_{3} / \mathrm{MAO}$, 主要产 物是三聚产物, $\mathrm{C}_{8}$ 化合物最多只有 $17 \%$. 然而, 邻位乙 基取代的配体催化体系 $17 / \mathrm{Cr}(\mathrm{acac})_{3} / \mathrm{MAO}$ 在相同条件 下却得到了 $63 \%$ 的 $\mathrm{C}_{8}$ 选择性.

从这些结果可以看出, 两个重要因素导致了乙烯四 聚向三聚催化的转变. 首先, 金属中心周围更大的空间 位阻会增加三聚倾向，这和中心金属立体空间的拥挤程 度(主要是邻位取代基的个数)有着直接的关联 ${ }^{[37]}$. 其次, 邻位取代基对金属的配位效应也有着不可忽视的影响. 从目前的结果来看, 在保持 $[\mathrm{PNP}] \mathrm{Cr}$ 四元环主体配位结 构的前提下, 只需要一个邻位甲氧基对金属中心的配位 即可, 过多的配位作用反而会引起主体结构的解体. 因 此, 这也就解释了为什么潜在配位基团的减少不会对结 果造成太大影响.

前面的机理已经阐述 ${ }^{[54]}, 1-\mathrm{C}_{6}$ 和 $1-\mathrm{C}_{8}$ 的形成都经过 了一个 $\mathrm{CrC}_{6}$ 七元环的中间态. 从动力学角度来看, 齐聚 选择性由 $\beta$-氢消除的速率 ${ }^{[57 \mathrm{a}]}$ (生成 $1-\mathrm{C}_{6}$ ) 和乙烯插入速 率(生成 $1-\mathrm{C}_{8}$ ) 的相对大小来决定. 对于上述邻位烷基取 代的配体, 铬活性中心周围的空间位阻可能会限制 $\mathrm{CrC}_{6}$ 扩环而形成对 $\beta$-氢消除有利的构象; 对于邻位甲 氧基取代的配体，甲氧基的配位可能会延缓或不利于下 一个乙烯分子的配位和插入. 因此, 这两种取代基团数 目的增加总体上使三聚催化为主导, 相应结果也验证了 这一点.

同年, Sasol 技术公司的 Blann 等 ${ }^{[38]}$ 主要研发人员继 续对 [PNP]配体 $\mathrm{P}$ 原子上芳基取代基的效应进行研究(图 6). 对于 $20 / \mathrm{CrCl}_{3}(\mathrm{THF})_{3} / \mathrm{MAO}$ 体系, 齐聚反应在 $65{ }^{\circ} \mathrm{C} 、$ $3.0 \mathrm{MPa}$ 压力下进行, 给出 $90 \% \mathrm{C}_{6}$ 馏分和 $0.5 \% \mathrm{C}_{10}$ 馏分. 与 $\mathrm{BP}$ 公司报道的邻位甲氧基配体的结果 $(7 \% \sim 29 \%)^{[19]}$ 相比, $\mathrm{C}_{10}$ 馏分降至很低. 降低温度至 $45{ }^{\circ} \mathrm{C}$ 但增加乙烯 压力至 $4.5 \mathrm{MPa}$, 催化剂的活性提高一个数量级 $(3.0 \times$ $\left.10^{5} \mathrm{~g} / \mathrm{g} \mathrm{Cr} \bullet \mathrm{h}\right)$, 同时 $1-\mathrm{C}_{6}(86.0 \%)$ 和 $1-\mathrm{C}_{8}$ 的选择性(10.5\%) 有所变化.

$\mathrm{P}$ 原子上芳基无任何取代的[PNP]配体催化剂似乎

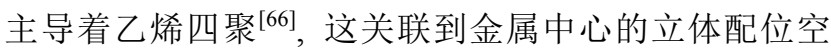
间作用，因此很有必要研究评估其它如邻位乙基和邻位 异丙基等取代基配体对催化剂的影响. 当磷原子上四个 芳环的邻位均分别被这两种烷基取代时(配体 2 和 21), $2 / \mathrm{CrCl}_{3}(\mathrm{THF})_{3} / \mathrm{MAO}$ 体系给出 $93.0 \%$ 的乙烯三聚选择性, $21 / \mathrm{CrCl}_{3}(\mathrm{THF})_{3} / \mathrm{MAO}$ 体系给出 $92.9 \%$ 的乙烯三聚选择 性，两者的结果基本上一样. 因此，不论是甲氧基或烷 基, 邻位取代基的存在则主导着乙烯的三聚催化. 值得 注意的是, $\mathrm{Cr}$ 源前驱体的选择 $\left[\mathrm{Cr}(\mathrm{acac})_{3}, \mathrm{CrCl}_{3}(\mathrm{THF})_{3}\right.$, $\mathrm{Cr}(\mathrm{EH})_{3}$ ] 对催化活性和选择性没有太大的影响, 催化活 性保持在 $10^{5} \mathrm{~g} /(\mathrm{g} \mathrm{Cr} \bullet \mathrm{h}), \mathrm{C}_{6}$ 的选择性均在 $85 \%$ 以上. 对 $2 / \mathrm{CrCl}_{3}(\mathrm{THF})_{3} / \mathrm{MAO}$ 体系, 甲苯和环己烷溶剂也给出相 近的催化结果; 使用不同的助剂, $2 / \mathrm{CrCl}_{3}$ (THF) $3 / \mathrm{MAO}$ (MMAO-3A, EAO/TMA)体系在甲苯溶剂中的结果也基 本上相近.

当改变芳环上邻位取代基的数目时(配体 17 和 22 26), 相应的催化体系中的 $\mathrm{C}_{6}$ 和 $\mathrm{C}_{8}$ 的选择性均有明显变 化. 对比配体 26 和配体 17, 芳环上邻位取代基的数目 分别为 2 和 $1, \mathrm{C}_{6}$ 选择性分别为 $59.1 \%$ 和 $27 \%, \mathrm{C}_{8}$ 的选择 性分别为 $34.1 \%$ 和 $63 \%$. 保留三个邻位甲基时, $22 / \mathrm{Cr}(\mathrm{acac})_{3} / \mathrm{MAO}$ 体系产物选择性发生了很大变化, $\mathrm{C}_{6}$ 馏分递减至 $42 \%$, 而 $\mathrm{C}_{8}$ 选择性提高到 $42 \%$, 其中 $\mathrm{C}_{6}$ 馏 分中 $1-\mathrm{C}_{6}$ 从 $99 \%$ 减少到 $82 \%$. 只有两个邻位甲基取代基 时(非对称配体 23 和对称配体 24), 催化利于四聚 $\left(\mathrm{C}_{6}\right.$ $17.1 \% \sim 29.8 \%, \mathrm{C}_{8} 47.6 \% \sim 66.0 \%$ ); 有两个甲基变为两 个乙基(对称配体 25)时，四聚催化结果类似 $\left(\mathrm{C}_{6} 38.3 \%\right.$, $\mathrm{C}_{8} 49.1 \%$ ). 当由 $\mathrm{N}$-异丙基交换为 $\mathrm{N}$-甲基时，则明显倾 向于三聚催化 $\left(\mathrm{C}_{6} 59.1 \%, \mathrm{C}_{8} 34.1 \%\right)$. 也就是说, $\mathrm{P}$ 上芳基 取代基的数目与 $\mathrm{N}$ 上取代基的大小对三聚和四聚的选 择性影响有一定的互补性. $\mathrm{P}$ 上芳基仅一个取代基(配体 17)时，四聚选择性为主导 $\left(\mathrm{C}_{6} 27.1 \%, \mathrm{C}_{8} 63.4 \%\right)$.

总体上来说，立体空间位阻不论来自 $\mathrm{P}$ 原子上或 $\mathrm{N}$ 原子上，最后均对中心金属的配位空间产生影响.

通过对 $\mathrm{P}$ 上芳基不同取代基的系统性研究，科学家 们大致可以确定乙烯催化三聚到四聚以及与之相反模 式的转换趋势. 但是反应条件如温度和压力的变化也对 这些转换有着很大的影响. 2006 年, 德国 ErlangenNürnberg 大学的 Wasserscheid 和 Sasol 技术公司的研究 人员 ${ }^{[67]}$ 对 9/Cr(acac) $3 / \mathrm{MAO}$ 以异丙苯做溶剂的催化体系 进行了动力学研究, 结果显示乙烯压力从 $2.0 \mathrm{MPa}$ 上升 至 4.5 MPa, 1- $\mathrm{C}_{8}$ 的产率会随着压力升高而上升. 产物的 分布特别是 $1-\mathrm{C}_{8}$ 依赖于乙烯浓度. 这个发现促使他们扩 大压力的研究范围 $\left(4.5 \mathrm{MPa}\right.$ 到 $10.0 \mathrm{MPa}$ )以检验 $1-\mathrm{C}_{8}$ 的 选择性是否可以更进一步提高. 同时针对反应是三聚还 是四聚, 温度也是一个非常重要的因素. 因此, 他们 


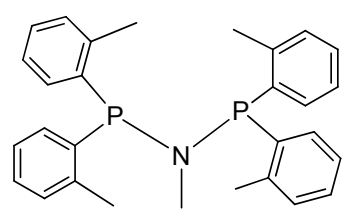

20

$20 / \mathrm{Cr}(\mathrm{acac})_{3} / \mathrm{MAO} /$ toluene $45^{\circ} \mathrm{C}, 4.5 \mathrm{MPa}$

Activity: $3.0 \times 10^{5} \mathrm{~g} /(\mathrm{g} \mathrm{Cr} \cdot \mathrm{h})$ Selectivity: (\%)

$\mathrm{C}_{6}\left(1-\mathrm{C}_{6}\right): 86.0(99.1)$

$\mathrm{C}_{8}\left(1-\mathrm{C}_{8}\right): 10.5$ (>99)

$\mathrm{C}_{10+}: 0.3$

PE: 3.3

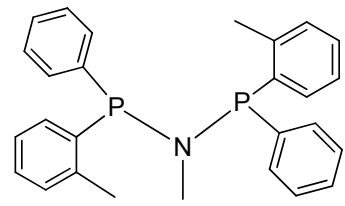

24

$24 / \mathrm{Cr}(\mathrm{acac})_{3} / \mathrm{MAO} /$ toluene $45^{\circ} \mathrm{C}, 4.5 \mathrm{MPa}$

Activity: $2.6 \times 10^{4} \mathrm{~g} /(\mathrm{g} \mathrm{Cr} \cdot \mathrm{h})$

Selectivity: (\%)

$\mathrm{C}_{6}\left(1-\mathrm{C}_{6}\right): 29.8(26.0)$

$\mathrm{C}_{8}\left(1-\mathrm{C}_{8}\right): 47.6(94.9)$

$\mathrm{C}_{10+}: 2.8$

PE: 9.0<smiles>CCc1ccccc1P(c1ccccc1)N(C)P(c1ccccc1CC)c1ccccc1CC</smiles>

21

21/Cr(acac) $)_{3} / \mathrm{MAO} /$ toluene $45^{\circ} \mathrm{C}, 4.5 \mathrm{MPa}$

Activity: $1.0 \times 10^{5} \mathrm{~g} /(\mathrm{g} \mathrm{Cr} \cdot \mathrm{h})$ Selectivity: (\%)

$\mathrm{C}_{6}\left(1-\mathrm{C}_{6}\right): 92.9$ (99.3)

$\mathrm{C}_{8}\left(1-\mathrm{C}_{8}\right): 2.7$ (93.8)

$\mathrm{C}_{10+}: 1.4$

PE: 2.4<smiles>Cc1ccccc1P(c1ccccc1)N(C)P(c1ccccc1C)c1ccccc1C</smiles>

22

22/ $\mathrm{Cr}$ (acac) $)_{3} / \mathrm{MAO} /$ toluene $45^{\circ} \mathrm{C}, 4.5 \mathrm{MPa}$

Activity: $9.7 \times 10^{4} \mathrm{~g} /(\mathrm{g} \mathrm{Cr} \cdot \mathrm{h})$ Selectivity: (\%)

$\mathrm{C}_{6}\left(1-\mathrm{C}_{6}\right): 41.5$ (81.9)

$\mathrm{C}_{8}\left(1-\mathrm{C}_{8}\right): 41.9$ (98.3)

$\mathrm{C}_{10+}: 1.2$

PE: 12.0<smiles>Cc1ccccc1P(c1ccccc1C)N(C)P(c1ccccc1)c1ccccc1</smiles>

23

23/Cr(acac) $)_{3} / \mathrm{MAO} /$ toluene $45^{\circ} \mathrm{C}, 4.5 \mathrm{MPa}$

Activity: $3.7 \times 10^{4} \mathrm{~g} /(\mathrm{g} \mathrm{Cr} \cdot \mathrm{h})$

Selectivity: (\%)

C6 (1-C6): 17.1 (55.3)

$\mathrm{C}_{8}\left(1-\mathrm{C}_{8}\right): 66.0(98.2)$

$\mathrm{C}_{10+}: 1.4$

PE: 8.3

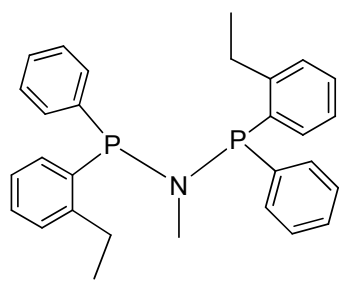

25

$25 / \mathrm{Cr}(\mathrm{acac})_{3} / \mathrm{MAO} /$ toluene $45^{\circ} \mathrm{C}, 4.5 \mathrm{MPa}$

Activity: $5.2 \times 10^{4} \mathrm{~g} /(\mathrm{g} \mathrm{Cr} \cdot \mathrm{h})$

Selectivity: (\%)

$\mathrm{C}_{6}\left(1-\mathrm{C}_{6}\right): 38.3(39.1)$

$\mathrm{C}_{8}\left(1-\mathrm{C}_{8}\right): 49.1$ (95.9)

$\mathrm{C}_{10+}: 2.0$

PE: 3.9<smiles>CCc1ccccc1P(c1ccccc1)N(C(C)C)P(c1ccccc1)c1ccccc1CC</smiles>

26

26/Cr(acac) $)_{3} / \mathrm{MAO} /$ toluene $45^{\circ} \mathrm{C}, 4.5 \mathrm{MPa}$

Activity: $1.1 \times 10^{5} \mathrm{~g} /(\mathrm{g} \mathrm{Cr} \cdot \mathrm{h})$

Selectivity: (\%)

$\mathrm{C}_{6}\left(1-\mathrm{C}_{6}\right): 59.1(94.1)$

$\mathrm{C}_{8}\left(1-\mathrm{C}_{8}\right): 34.1$ (>99)

$\mathrm{C}_{10+}: 1.7$

PE: 2.8

图 6 [PNP]配体 20 26 的结构式以及催化结果

Figure 6 Schematic structures of [PNP] ligands $\mathbf{2 0} \sim \mathbf{2 6}$ and catalytic results<smiles>c1ccc(P(c2ccccc2)N(C2CCCc3ccccc32)P(c2ccccc2)c2ccccc2)cc1</smiles>

27

27/Cr(acac) $)_{3} / \mathrm{MMAO}-3 \mathrm{~A} /$ cyclohexane $40{ }^{\circ} \mathrm{C}, 7.0 \mathrm{MPa}$

Activity: $3.6 \times 10^{5} \mathrm{~g} /(\mathrm{g} \mathrm{Cr} \cdot \mathrm{h})$

Selectivity: (\%)

$\mathrm{C}_{6}\left(1-\mathrm{C}_{6}\right): 17.7$ (74.9)

$\mathrm{C}_{8}\left(1-\mathrm{C}_{8}\right): 75.2(99.2)$

$\mathrm{C}_{10+}: \mathrm{ndr}$

PE: ndr<smiles>c1ccc(P(c2ccccc2)N(C2CCCC2)P(c2ccccc2)c2ccccc2)cc1</smiles>

28

$28 / \mathrm{CrCl}_{3}(\mathrm{THF})_{3} / \mathrm{MAO} / \mathrm{TCE} /$ cyclohexane

$50{ }^{\circ} \mathrm{C}, 5.0 \mathrm{MPa}$

Activity: $2.4 \times 10^{5} \mathrm{~g} /(\mathrm{g} \mathrm{Cr} \cdot \mathrm{h})$

Selectivity: (\%)

$1-\mathrm{C}_{6}: 15.1$

$1-\mathrm{C}_{8}: 72.7$

$\mathrm{C}_{10+}: 4.3$

PE: ndr

图 7 [PNP]配体 $\mathbf{2 7}, \mathbf{2 8}$ 的结构式以及催化结果

Figure 7 Schematic structures of [PNP] ligands 27, 28 and catalytic results

选择配体 1,2,3,4-四氢萗胺-双(二苯基膦)(27)(图 7), 并 按温度从 40 到 $80{ }^{\circ} \mathrm{C}$ 变化, 以确保同等级数时催化剂的 效率, 便于对选择性数据进行比较, 进而深入地了解
$1-\mathrm{C}_{8}$ 形成的反应机理和决速步参数.

27/Cr(acac) $3 / \mathrm{MMAO}-3 \mathrm{~A}$ 在环已烷溶剂中 $40 \sim$ $80{ }^{\circ} \mathrm{C} 、 4.5 \sim 10.0 \mathrm{MPa}$ 乙烯压力下, $1-\mathrm{C}_{6}$ 和 $1-\mathrm{C}_{8}$ 是主产 
物, 总产率在 $90 \%$ 左右, 基本恒定. 在整个实验设置中, 总产率仅有 3\% 4\%的差异. 相应 $\mathrm{C}_{6}$ 和 $\mathrm{C}_{8}$ 液体产物的 总产率大约为 $95 \%$. 该反应的主要副产物是亚甲基环戊 烷和甲基环戊烷，比例为 $1: 1$. 当温度保持在 $40{ }^{\circ} \mathrm{C}$ 时, 催化活性为 $10^{5} \mathrm{~g} /(\mathrm{g} \mathrm{Cr} \bullet \mathrm{h}), 1$-辛烯的选择性均大于 $72 \%$; 温度保持 $60{ }^{\circ} \mathrm{C}$, 随着乙烯压力的不断升高, $1-\mathrm{C}_{6}$ 的选择 性呈下降趋势, 由 $32.1 \%$ 逐渐降为 $25.7 \%$, 而 $1-\mathrm{C}_{8}$ 的选 择性不断上升, 由 $58.8 \%$ 上升至 $65.4 \%$, 其催化活性变 化不大; 当温度保持在 $80{ }^{\circ} \mathrm{C}$ 时, 催化活性较 $40{ }^{\circ} \mathrm{C}$ 时下 降了一个数量级, $1-\mathrm{C}_{6}$ 的选择性明显升高, 大于 $40 \%$, $1-\mathrm{C}_{8}$ 的选择性则降为 $50 \%$ 以下.

随着压力的变化, 三个主产物 $\left(1-\mathrm{C}_{8}, 1-\mathrm{C}_{6}\right.$ 和环 $\mathrm{C}_{6}$ 物 种)的分布也发生改变, 该结果与 Overett 等提出的金属 环化机理 ${ }^{[56]}$ 很好地吻合. 值得注意的是, 在 $60{ }^{\circ} \mathrm{C}$ 下, 压力从 $4.5 \mathrm{MPa}$ 上升至 $8.0 \mathrm{MPa}$, 生成的 1- $\mathrm{C}_{8}$ 仅增加了 $6 \%$; 进一步升高压力到 $10.0 \mathrm{MPa}, 1-\mathrm{C}_{8}$ 产率没有变化. 正如预期结果, 液相中乙烯的浓度超过该压力范围一倍 时, 选择性会有明显差异. 然而, $1-\mathrm{C}_{8}$ 的最大收率似乎 达到一定的值后便不再上升. 相比而言, $80{ }^{\circ} \mathrm{C}$ $(41.5 \% \sim 50.8 \%)$ 时的 $1-\mathrm{C}_{8}$ 选择性随压力变化较 $40{ }^{\circ} \mathrm{C}$ ( $72.7 \% \sim 74.6 \%)$ 时大, 但都处在较低的值.

$\mathrm{C}_{6}$ 环类副产物的形成在该压力范围内似乎不依赖 于乙烯的浓度. 例如, 在 $60{ }^{\circ} \mathrm{C}$ 下, 压力从 $4.5 \mathrm{MPa}$ 到 $10.0 \mathrm{MPa}$ 变化, 亚甲基环戊烷和甲基环戊烷一直保持在 $2.8 \% \sim 2.9 \%$ 的水平.

常压下, 温度的升高通常会引起乙烯浓度的降低, 在较高压力下亦是如此. 例如在 $4.5 \mathrm{MPa}$ 下, 温度从 $40 \sim 80{ }^{\circ} \mathrm{C}$, 乙烯浓度 $X_{\text {ethylene }}$ 则明显从 0.52 降至 0.35 . 调节温度和压力, 也可以使得乙烯的浓度大致相同, 如 在 $40{ }^{\circ} \mathrm{C}$ 和 $4.5 \mathrm{MPa}$ 下 $X_{\text {ethylene }}$ 为 0.52 , 在 $80{ }^{\circ} \mathrm{C}$ 和 7.0 $\mathrm{MPa}$ 下 $X_{\text {ethylene }}$ 为 0.53 . 当 $X_{\text {ethylene }}=0.52 \sim 0.53$ 时, $1-\mathrm{C}_{6}$ 的总选择性从 $40{ }^{\circ} \mathrm{C}$ 时的 $16.3 \%$ 上升到 $80{ }^{\circ} \mathrm{C}$ 时的 $41.6 \%$, 而 $1-\mathrm{C}_{8}$ 的选择性则从 $40{ }^{\circ} \mathrm{C}$ 时的 $72.7 \%$ 下降到 $80{ }^{\circ} \mathrm{C}$ 时的 $46.4 \%$. 这些结果表明 $\mathrm{C}_{6}$ 至 $\mathrm{C}_{8}$ 的动力学转化 事实上具温度依赖性, 主要是由温度决定. 相对而言, 压力对 $\mathrm{C}_{6}$ 选择性的影响小于对 $\mathrm{C}_{8}$ 的影响. 因此, 依据 这些研究我们可以推测, $\mathrm{CrC}_{8}$ 环中间体的稳定性比较容 易受反应温度的影响. 但是提高乙烯浓度, $\mathrm{CrC}_{8}$ 浓度会 限制在某一值而不会一直提高. 通过调整反应参数的方 式来对 $1-\mathrm{C}_{8}$ 的形成进行进一步优化以及对副产物进行 抑制似乎是不大可行的.

同年, 大庆石油学院的姜涛等 ${ }^{[6]}$ 研究了 $N$-环戊基 [PNP] 配体的 $28 / \mathrm{CrCl}_{3}(\mathrm{THF})_{3} / \mathrm{MAO} / \mathrm{TCE}$ (四氯乙烷) 催化 体系(图 7). 他们系统地考察了反应温度、压力、物质的 量比( $\mathrm{Al} / \mathrm{Cr}, \mathrm{TCE} / \mathrm{Cr}, 28 / \mathrm{Cr}$ )、反应时间以及助剂种类对催
化活性和 1- $\mathrm{C}_{8}$ 选择性的影响.

选择 $28 / \mathrm{CrCl}_{3}(\mathrm{THF})_{3} / \mathrm{MAO} / \mathrm{TCE}(2: 1: 300: 1)$ 四 组分体系, 保持 $5.0 \mathrm{MPa}$ 乙烯压力不变, 反应时间 30 min, 随着反应温度升高, 催化活性呈先升高后降低趋 势, 在 $50{ }^{\circ} \mathrm{C}$ 时最大, 为 $2.3 \times 10^{5} \mathrm{~g} /(\mathrm{g} \mathrm{Cr} \bullet \mathrm{h}) ; 1-\mathrm{C}_{8}$ 的选择 性也是先升高后降低, 在 $50{ }^{\circ} \mathrm{C}$ 时最大, 为 $72.7 \%$. 因此 最佳反应温度可以选择 $50{ }^{\circ} \mathrm{C}$.

保持 $50{ }^{\circ} \mathrm{C}$ 和反应时间 $30 \mathrm{~min}$, 上述催化体系随着 反应压力的升高, 催化活性不断升高, 在 $6.0 \mathrm{MPa}$ 时最 大 $\left[3.1 \times 10^{5} \mathrm{~g} /(\mathrm{g} \mathrm{Cr} \bullet \mathrm{h})\right] ; 1-\mathrm{C}_{8}$ 的选择性也不断升高, 在 6.0 MPa 时达到最大 $(73.1 \%)$. 因此最佳反应压力可以选 择 $5.0 \mathrm{MPa}$.

在 $50{ }^{\circ} \mathrm{C} 、 5.0 \mathrm{MPa}$ 条件下, 保持 $\mathbf{2 8} / \mathrm{CrCl}_{3}(\mathrm{THF})_{3} /$ $\mathrm{TCE}$ 为 $2: 1: 1$, 反应时间 $30 \mathrm{~min}$, 调节 $\mathrm{Al} / \mathrm{Cr}$ 比 75 至 400 , 催化活性先升高后降低, 当 $\mathrm{Al} / \mathrm{Cr}$ 为 300 时达到最 大 $\left[2.4 \times 10^{5} \mathrm{~g} /(\mathrm{g} \mathrm{Cr} \cdot \mathrm{h})\right] ; 1-\mathrm{C}_{8}$ 选择性也先升高后降低, 当 $\mathrm{Al} / \mathrm{Cr}$ 为 300 时达到最大 $(72.7 \%)$. 紧接着, 在 $28 / \mathrm{CrCl}_{3}(\mathrm{THF})_{3} / \mathrm{MAO}$ 为 $2: 1: 300$ 和反应时间 $30 \mathrm{~min}$ 下, 调节 $\mathrm{TCE} / \mathrm{CrCl}_{3}(\mathrm{THF})_{3}$ 比从 0 至 10 , 催化活性不断 降低 $\left[\right.$ 从 $3.6 \times 10^{5} \mathrm{~g} /(\mathrm{g} \mathrm{Cr} \bullet \mathrm{h})$ 降至 $\left.0.7 \times 10^{5} \mathrm{~g} /(\mathrm{g} \mathrm{Cr} \bullet \mathrm{h})\right]$, 而 $1-\mathrm{C}_{8}$ 的选择性略呈升高趋势(从 $71.8 \%$ 至 $74.9 \%$ ). 而在 $\mathrm{CrCl}_{3}(\mathrm{THF})_{3} / \mathrm{MAO} / \mathrm{TCE}$ 为 $1: 300: 1$ 和 $30 \mathrm{~min}$ 内, 随着 $\mathrm{PNP} / \mathrm{CrCl}_{3}(\mathrm{THF})_{3}$ 比从 1 至 4 , 催化活性不断降低 $[$ 从 $3.0 \times 10^{5} \mathrm{~g} /(\mathrm{g} \mathrm{Cr} \bullet \mathrm{h})$ 降至 $\left.0.3 \times 10^{5} \mathrm{~g} /(\mathrm{g} \mathrm{Cr} \bullet \mathrm{h})\right]$, 但是 $1-\mathrm{C}_{8}$ 的选择性略呈升高趋势( $69.3 \% \sim 71.7 \%$ ). 对于 $\mathbf{2 8} / \mathrm{CrCl}_{3}$ $(\mathrm{THF})_{3} / \mathrm{MAO} / \mathrm{TCE}$ 为 $2: 1: 300: 1$ 体系, 在 $50{ }^{\circ} \mathrm{C} 、 5.0$ $\mathrm{MPa}$ 条件下, 监测反应过程中乙烯的流速及 1-辛烯的选 择性, 发现反应 $40 \mathrm{~min}$ 以后催化活性开始降低. 经推测 可能是催化剂失活, 但在此期间 1- $\mathrm{C}_{8}$ 的选择性基本保持 不变. 在相同条件下保持 $30 \mathrm{~min}$ 反应时间, 助剂种类考 察发现, 催化活性和 1- $\mathrm{C}_{8}$ 选择性在一定程度上受助剂影 响(MAO $>$ EAO $>$ TEA).

[PNP] 配体 P 原子上芳基中含氧基团易于与铬配位, 影响到齐聚选择性 ${ }^{[65]}$. 那么, $\mathrm{N}$ 原子上的含氧基团会不 会参与到中心铬的配位呢? 为此, 2006 年 Bercaw 等 ${ }^{[69]}$ 合成了一系列氮原子上含有醚基的 PNP 配体 $(29,30,31$, 32)(图 8), 并与 $\mathrm{CrCl}_{3}(\mathrm{THF})_{3}$ 反应制备相应的金属化合 物. 化合物 $\left\{[29] \mathrm{CrCl}_{2}(\mu-\mathrm{Cl})\right\}_{2}$ 是二聚体, 在固体状态 $\mathrm{N}$ 原子上的含氧基团并没有与中心铬配位.

$\left\{[29(30 、 31 、 32)] \mathrm{CrCl}_{2}(\mu-\mathrm{Cl})\right\}_{2} / \mathrm{MAO}$ 在氯苯溶剂中 室温、 $0.1 \mathrm{MPa}$ 乙烯压力下可选择性地催化乙烯三聚 $\left(\mathrm{C}_{6}\right.$ $45 \% \sim 66 \%, \mathrm{C}_{8} 16 \% \sim 34 \%, \mathrm{C}_{10^{+}} 1 \% \sim 40 \%$, PE $0.1 \% \sim$ $6.0 \%)$, 催化活性比较低 $\left[3.61 \times 10^{2} \sim 6.24 \times 10^{3} \mathrm{~g} /(\mathrm{g}\right.$ $\mathrm{Cr} \cdot \mathrm{h})]$. $\mathrm{N}$ 原子上取代基给电子体的长度和刚性会大大 影响催化剂的活性和稳定性. $\left\{[32] \mathrm{CrCl}_{2}(\mu-\mathrm{Cl})\right\}_{2} / \mathrm{MAO}$ 


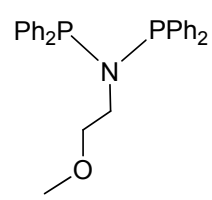

29

$\mathrm{LCrCl}_{3} / \mathrm{MAO} / \mathrm{C}_{6} \mathrm{H}_{5} \mathrm{Cl}$ $25^{\circ} \mathrm{C}, 0.1 \mathrm{MPa}$ Activity: $3.6 \times 10^{2} \mathrm{~g} /(\mathrm{g} \mathrm{Cr} \cdot \mathrm{h})$ Activity: $4.0 \times 10^{2} \mathrm{~g} /(\mathrm{g} \mathrm{Cr} \cdot \mathrm{h})$ Selectivity: (\%) $\mathrm{C}_{6}\left(1-\mathrm{C}_{6}\right): 61$ (83) $\mathrm{C}_{8}\left(1-\mathrm{C}_{8}\right): 31(>90)$ $\mathrm{C}_{10+}: 2$ PE: 6<smiles>COc1ccccc1N(c1ccccc1)c1ccccc1</smiles>

31 $\mathrm{LCrCl}_{3} / \mathrm{MAO} / \mathrm{C}_{6} \mathrm{H}_{5} \mathrm{Cl}$ $25^{\circ} \mathrm{C}, 0.1 \mathrm{MPa}$ Activity: $9.2 \times 10^{2} \mathrm{~g} /(\mathrm{g} \mathrm{Cr} \cdot \mathrm{h})$ Selectivity: $(\%)$ $\mathrm{C}_{6}\left(1-\mathrm{C}_{6}\right): 66$ (91) $\mathrm{C}_{8}\left(1-\mathrm{C}_{8}\right): 27(97)$ $\mathrm{C}_{10+}: 7$ PE: 0.3<smiles>CCc1ccccc1CN(Pc1ccccc1)Pc1ccccc1</smiles>

33

$\mathrm{LCrCl}_{3} / \mathrm{MAO} / \mathrm{C}_{6} \mathrm{H}_{5} \mathrm{Cl}$

$25^{\circ} \mathrm{C}, 0.4 \mathrm{MPa}$

Activity: $2.4 \times 10^{5} \mathrm{~g} /(\mathrm{g} \mathrm{Cr} \cdot \mathrm{h})$

Selectivity: $(\%)$

$\mathrm{C}_{6}\left(1-\mathrm{C}_{6}\right): 40(91)$

$\mathrm{C}_{8}\left(1-\mathrm{C}_{8}\right): 34(93)$

$C_{10+}: 25$

PE: 0.2

图 8 [PNP]配体 29 33 的结构式以及催化结果

Figure 8 Schematic structures of [PNP] ligands $29 \sim 33$ and catalytic results

的活性相对较高, 而且活性可持续达 $2 \mathrm{~h}$, 此后缓慢下 降. 与 $1 / \mathrm{CrCl}_{3}(\mathrm{THF})_{3} / \mathrm{MAO}$ 催化体系对照, 后者的稳定 性稍差(不能持续 $20 \mathrm{~min}$ ). $\left\{[\mathbf{3 2}] \mathrm{CrCl}_{2}(\mu-\mathrm{Cl})\right\}_{2} / \mathrm{MAO}$ 体系 的活性考察表明, 氯苯溶剂显示了更高的活性和稳定 性, 并只产生少量聚乙烯; 甲苯溶剂只给出低活性, 产 生很多 PE; 1,2-二氯苯溶剂的催化体系稳定性高, 然而 活性稍低. $\left\{[9 \text { 或 33 }] \mathrm{CrCl}_{2}(\mu-\mathrm{Cl})\right\}_{2} / \mathrm{MAO}$ 体系的活性考察 表明, 氯苯较甲苯溶剂可以提高催化剂的稳定性、活性 以及 $1-\mathrm{C}_{6}$ 和 $1-\mathrm{C}_{8}$ 的选择性, 还可以减少 $\mathrm{PE}$ 的量. 目前 尚不清楚氯苯的有效影响是由于氯原子的弱溶解性还

是强极性.

对 $\left\{[32] \mathrm{CrCl}_{2}(\mu-\mathrm{Cl})\right\}_{2} / \mathrm{MAO}$ 体系进行压力对反应活 性、选择性和产品分布影响的实验考察表明, 在所测试 的压力范围内, 压力越大, 乙烯浓度越高, 则 $1-\mathrm{C}_{8}$ 和 $1-\mathrm{C}_{6}$ 的比例线性增加, 但是相应的 $\mathrm{C}_{6}$ 或 $\mathrm{C}_{8}$ 中的选择性 变化不大.

对混合产物进行仔细分析, $\mathrm{C}_{10}$ 及 $\mathrm{C}_{10^{+}}$的形成应该 是 $\alpha$-烯烃插入铬碳环中间体中形成. 此外, 在短时间内 $1-\mathrm{C}_{6}$ 和 $1-\mathrm{C}_{8}$ 的浓度低, 因而高碳数的齐聚物的量急剧减 少. 一些钽、钢和铬的三聚反应研究表明, 金属辅助协 调的 3,7 氢转移更易形成 $\mathrm{CrC}_{6}$ 环中间体，而不是逐步消 除 $\alpha$-氢和还原消除释放 1- $\mathrm{C}_{6}{ }^{[57]}$.

同时 Bercaw 等 ${ }^{[34]}$ 合成了苯环中氛代甲氧基和氛代 甲硫基的 PNP 配体 34 和 35(图 9), 再分别与 $\mathrm{CrPh}_{3}(\mathrm{THF})_{3}$ 反应生成化合物 $[\mathbf{3 4}] \mathrm{CrPh}_{3}$ 和 $[35] \mathrm{CrPh}_{3} .34$ 与 $\mathrm{CrCl}_{3}(\mathrm{THF})_{3}$ 反应生成化合物 $[34] \mathrm{CrCl}_{3}$, 其进一步与 $[34] \mathrm{CrPh}_{3}$ 反应生成 $[34] \mathrm{CrPh}_{2} \mathrm{Cl}$. 化合物 $[34] \mathrm{CrPh}_{3}$ 分别 在助剂 MAO, $\left[\mathrm{H}\left(\mathrm{OEt}_{2}\right)_{2}\right]^{+}\left[\mathrm{BAr}_{4}^{\mathrm{F}}\right]^{-},\left[\mathrm{Me}_{2} \mathrm{PhNH}\right]^{+}$$\left[\mathrm{B}\left(\mathrm{C}_{6} \mathrm{~F}_{5}\right)_{4}\right]^{-}$的作用下显示出一定的乙烯齐聚催化活性, 但是受溶剂的影响很大. 化合物 [34] $\mathrm{CrPh}_{2} \mathrm{Cl}$ 在助剂 $\mathrm{Na}^{+}$ $\left[\mathrm{BAr}^{\mathrm{F}}{ }_{4}\right]^{-}$的作用下也具有催化活性. 他们认为这些催化 结果可比于 Wass 等 ${ }^{[19]}$ 报道的 $1 / \mathrm{CrCl}_{3}(\mathrm{THF})_{3} / \mathrm{MAO}$ 体系. 他们也对 $\mathbf{3 5} / \mathrm{CrCl}_{3}(\mathrm{THF})_{3} / \mathrm{MAO}$ 体系进行了初步的催化 实验, 结果是没有活性, 原因可能是没有形成活性催化 剂. 化合物[35] $\mathrm{CrPh}_{3}$ 未进行催化活性测试.

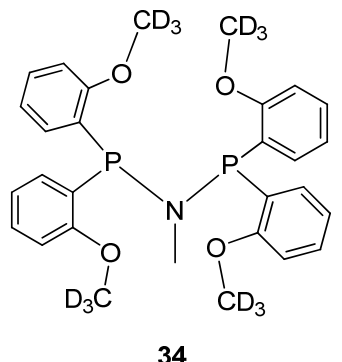

34

图 9 [PNP]配体 34 和 35 的结构式

Figure 9 Schematic structures of [PNP] ligands $\mathbf{3 4}$ and $\mathbf{3 5}$

[PNP]配体辅助的铬基催化剂因其独特的乙烯选择 性齐聚的调控能力而倍受关注. 随着对 $\mathrm{P}$ 上取代基作用 的剖析 ${ }^{[37]}, \mathrm{N}$ 原子上取代基的广泛性研究有待进一步深 入. 因此, 2007 年, Blann 等 ${ }^{[38]}$ 报道了一系列氮原子上含 有不同取代基的[PNP] 配体(图 10), 系统地考察了配体 结构对催化活性和选择性的影响.

36/ $\mathrm{Cr}(\mathrm{EH})_{3} / \mathrm{MAO}$ 体系在甲苯溶剂中 $65{ }^{\circ} \mathrm{C} 、 3.0$ $\mathrm{MPa}$ 乙烯压力下给出 $\mathrm{C}_{6}\left(17.7 \%\right.$, 其中 1- $\left.\mathrm{C}_{6} 97.5 \%\right)$ 和 $\mathrm{C}_{8}$ (16.4\%, 其中 $\left.1-\mathrm{C}_{8} 97.5 \%\right)$ 以及 $\mathrm{C}_{10 \sim 14}(32.6 \%) 、 \mathrm{C}_{16+}$ (20.2\%)和 PE (2.1\%)产物, 没有明显的选择性, 催化活 
<smiles>Pc1ccccc1</smiles>

36

$36 / \mathrm{Cr}(\mathrm{EH})_{3} / \mathrm{MAO} /$ toluene $65^{\circ} \mathrm{C}, 3.0 \mathrm{MPa}$ Activity: $1.3 \times 10^{4} \mathrm{~g} /(\mathrm{g}$ Cr.h) Selectivity: (\%) $\mathrm{C}_{6}\left(1-\mathrm{C}_{6}\right): 17.7$ (97.5) $\mathrm{C}_{8}\left(1-\mathrm{C}_{8}\right): 16.4$ (97.5) $\mathrm{C}_{10+}: 52.8$ PE: 2.1

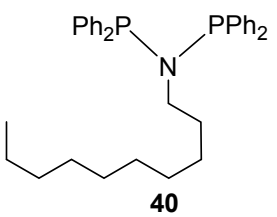

$40 / \mathrm{Cr}(\mathrm{acac})_{3} / \mathrm{MAO} /$ toluene $65{ }^{\circ} \mathrm{C}, 3.0 \mathrm{MPa}$

Activity: $5.0 \times 10^{4} \mathrm{~g} /(\mathrm{g}$ Cr.h) Selectivity: (\%) $\mathrm{C}_{6}\left(1-\mathrm{C}_{6}\right): 25.4(54.9)$ $\mathrm{C}_{8}\left(1-\mathrm{C}_{8}\right): 59.0$ (96.5) $C_{10+}: 12,5$ PE: 0.7

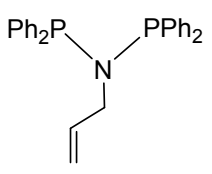

37

$37 / \mathrm{Cr}(\mathrm{EH})_{3} / \mathrm{MAO} /$ toluene $65^{\circ} \mathrm{C}, 3.0 \mathrm{MPa}$

Activity: $7.4 \times 10^{3} \mathrm{~g} /(\mathrm{g}$ Cr.h)

Selectivity: (\%)

$\mathrm{C}_{6}\left(1-\mathrm{C}_{6}\right): 22.2$ (53.9)

$\mathrm{C}_{8}\left(1-\mathrm{C}_{8}\right): 53.6(96.6)$

$\mathrm{C}_{10+}: 11.8$

PE: 9.8

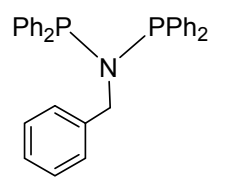

41

$41 / \mathrm{CrCl}_{3}(\mathrm{THF})_{3} / \mathrm{MAO} /$ toluene $65^{\circ} \mathrm{C}, 3.0 \mathrm{MPa}$

Activity: $1.3 \times 10^{4} \mathrm{~g} /(\mathrm{g}$ Cr.h)

Selectivity: (\%)

$\mathrm{C}_{6}\left(1-\mathrm{C}_{6}\right): 28.0(61.7)$

$\mathrm{C}_{8}\left(1-\mathrm{C}_{8}\right): 60.2$ (98.1)

$\mathrm{C}_{10+}: 5.7$

PE: 3.7

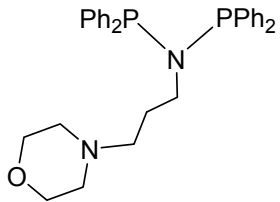

38

38/Cr(acac) $)_{3} / \mathrm{MAO} /$ toluene $65^{\circ} \mathrm{C}, 3.0 \mathrm{MPa}$

Activity: $1.3 \times 10^{4} \mathrm{~g} /(\mathrm{g}$ Cr.h)

Selectivity: (\%)

$\mathrm{C}_{6}\left(1-\mathrm{C}_{6}\right): 19.2$ (55.7)

$\mathrm{C}_{8}\left(1-\mathrm{C}_{8}\right): 45.4$ (96.4)

$\mathrm{C}_{10+}: 9.1$

PE: 23.9

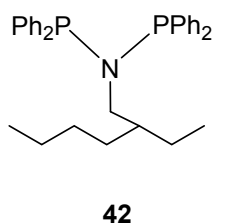

$42 / \mathrm{Cr}(\mathrm{acac})_{3} / \mathrm{MAO} /$ toluene $65^{\circ} \mathrm{C}, 30$ bar

Activity: $4.9 \times 10^{4} \mathrm{~g} /(\mathrm{g} \mathrm{Cr} . \mathrm{h})$

Selectivity: (\%)

$\mathrm{C}_{6}\left(1-\mathrm{C}_{6}\right): 27.3(66.2)$

$\mathrm{C}_{8}\left(1-\mathrm{C}_{8}\right): 57.3(98.0)$

$\mathrm{C}_{10+}: 13.1$

PE: 0.8

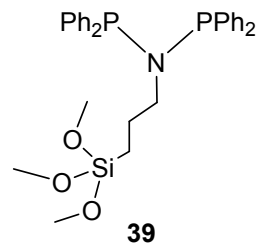

$39 / \mathrm{Cr}(\mathrm{acac})_{3} / \mathrm{MAO} /$ toluene $65^{\circ} \mathrm{C}, 3.0 \mathrm{MPa}$

Activity: $4.3 \times 10^{4} \mathrm{~g} /(\mathrm{g}$ Cr.h $)$

Selectivity: (\%)

$\mathrm{C}_{6}\left(1-\mathrm{C}_{6}\right): 23.5$ (54.7)

$\mathrm{C}_{8}\left(1-\mathrm{C}_{8}\right): 55.2(96.4)$

$\mathrm{C}_{10+}: 18.4$

PE: 0.5

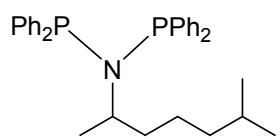

43

$43 / \mathrm{Cr}(\mathrm{acac})_{3} / \mathrm{MAO} /$ toluene $65^{\circ} \mathrm{C}, 3.0 \mathrm{MPa}$

Activity: $1.2 \times 10^{4} \mathrm{~g} /(\mathrm{g} \mathrm{Cr} . \mathrm{h})$

Selectivity: (\%)

$\mathrm{C}_{6}\left(1-\mathrm{C}_{6}\right): 36.2(82.1)$

$\mathrm{C}_{8}\left(1-\mathrm{C}_{8}\right): 57.7$ (99.2)

$\mathrm{C}_{10+}: 4.1$

PE: 1.8

图 10 [PNP]配体 36 43 的结构式以及催化结果

Figure 10 Schematic structures of [PNP] ligands $36 \sim 43$ and catalytic results

性为 $1.30 \times 10^{4} \mathrm{~g} /(\mathrm{g} \mathrm{Cr} \bullet \mathrm{h})$. 氮原子上含有一个甲基取代 基的配体 $6 / \mathrm{CrCl}_{3}(\mathrm{THF})_{3} / \mathrm{MAO}$ 体系给出 $\mathrm{C}_{8}$ 的选择性为 $59 \%$ (其中 $1-\mathrm{C}_{8} 94 \%$ ), $\mathrm{C}_{6}$ 的选择性为 $25 \%$ (其中 $1-\mathrm{C}_{6}$ $39 \%$ ). 配体 36 的 $\mathrm{N}$ 原子上是氢质子，可能在 $\mathrm{MAO}$ 活化 的过程中发生反应，导致[PNP]配位方式的变化，相关 的配位化学也已经报道 ${ }^{[45]}$.

在[PNP]配体的 $\mathrm{N}$ 原子上引入烯丙基(37)、吗啉烃 基(38)、三甲氧基硅烷基(39), 相应地 37/ $\mathrm{Cr}(\mathrm{EH})_{3} / \mathrm{MAO}$ 体系给出选择性的产物 $\left[\mathrm{C}_{6} \quad 22.2 \% \quad\left(1-\mathrm{C}_{6} 53.9 \%\right), \mathrm{C}_{8}\right.$ $53.6 \%\left(1-\mathrm{C}_{8} 96.6 \%\right), \mathrm{C}_{10-14} 6.3 \%, \mathrm{C}_{16+} 4.9 \%$, PE 9.8\%], 但活性较低 $\left[7.4 \times 10^{3} \mathrm{~g} /(\mathrm{g} \mathrm{Cr} \bullet \mathrm{h})\right]$. 在相同条件下, 38 $/ \mathrm{CrCl}_{3}(\mathrm{THF})_{3} / \mathrm{MAO}$ 体系给出的产物也具有一定的选 择性 $\left[\mathrm{C}_{6}\right.$ 19.2\% (1- $\left.\mathrm{C}_{6} 55.7 \%\right), \mathrm{C}_{8} 45.4 \%\left(1-\mathrm{C}_{8}\right.$ 96.4\%), $\mathrm{C}_{10 \sim 14} 6.3 \%, \mathrm{C}_{16+} 2.8 \%$, PE $23.9 \%$ ], 活性为 $1.29 \times 10^{4}$ $\mathrm{g} /(\mathrm{g} \mathrm{Cr} \bullet \mathrm{h})$. 在这两个反应体系中都有一定量的 PE 生成, 但产物的选择性并未受取代基的影响. $39 / \mathrm{CrCl}_{3}$ $(\mathrm{THF})_{3} / \mathrm{MAO}$ 体系给出的产物中 $\mathrm{PE}$ 含量非常少 $\left[\mathrm{C}_{6}\right.$

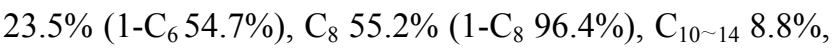
$\mathrm{C}_{16+} 9.6 \%$, PE 0.5\%], 活性为 $4.25 \times 10^{4} \mathrm{~g} /(\mathrm{g} \mathrm{Cr} \bullet \mathrm{h})$. 在这 三个体系中, $\alpha$-烯烃的总选择性为 $54 \%$, 活性 $4.0 \times 10^{4}$ $\mathrm{g} /(\mathrm{g} \mathrm{Cr} \bullet \mathrm{h})$. 配体 37 涉及的体系中 PE 比例明显升高, 可
能原因是 $\mathrm{N}$ 上取代基中的不饱和键参与到金属环中间 体的扩环反应，因此反应过程中活性齐聚催化剂的浓度 明显减小. 含配体 38 的体系可能是吗啉基团的三级胺 与铬中心发生配位作用, 导致 PE 的异常升高.

比较 $\mathrm{N}$ 原子上分别被甲基、戊基、癸基取代的 PNP 配体 6, 7 和 40, 当取代基链长增加四个碳原子(7), 催化 反应中 $\alpha$-烯烃的总选择性明显提高 $(65.3 \%$ 到 69.9\%), 而 再增加五个碳原子 $(\mathbf{4 0})$, 影响则很小 $(70.9 \%)$, 其中 $\mathrm{C}_{6}$ 和 $\mathrm{C}_{8}$ 的比例基本上保持不变, 但 $\mathrm{C}_{6}$ 中 $1-\mathrm{C}_{6}$ 的比例提高 (从 39.4\%到 54.7\%和 54.9\%). 在芳基取代的配体 41 包 含的体系中, 1- $\mathrm{C}_{6}$ 的选择性有所提高. 2-乙基已基取代的 配体 42 与甲基取代的 9 相比, 催化结果中 $1-\mathrm{C}_{6}$ 在 $\mathrm{C}_{6}$ 中的比例由 $39 \%$ 直接增加到 $66.2 \% .41$ 和 42 都含有 $\beta$ 支链, 但是 1- $\mathrm{C}_{6}$ 的选择性基本相同. 而 9 含有 $\alpha$-支链, 相应地 1- $\mathrm{C}_{6}$ 的选择性超过 $86.5 \%, \alpha$-烯烃的选择性达到 $88.4 \%$. 增加 $\alpha$-碳上支链的长度, 如配体 $43, \mathrm{C}_{6}$ 中 $1-\mathrm{C}_{6}$ 的选择性 $(82.1 \%)$ 和 $\alpha$-烯烃的选择性(84.7\%)均较佳. 由 此可以看出, $\mathrm{N}$ 上取代基的 $\alpha$-支链效应较 $\beta$-支链效应对 上述两种催化性能的影响要显著.

从 $N$-甲基配体(6)到 $N$-乙基配体(44)(图 11), 再到异 丙基配体(9), 它们与 $\mathrm{Cr}(\mathrm{acac})_{3} / \mathrm{MMAO}-3 \mathrm{~A}$ 组成的体系 
的催化结果显示, $\alpha$-烯烃的总选择性逐步增加(从 $65.3 \%$ 到 $68.42 \%$ 再到 $79.4 \%), \mathrm{C}_{16}$ 和 $\mathrm{PE}$ 的比例则显著降低. 同 是含 $\alpha$-支链的 $N$-环已基取代的配体 8 和 $\mathrm{N}$-异丙基配体 9 体系的催化结果相似.

对于系列 $N$-取代基的[PNP]配体， $\mathrm{N}$ 上取代基的立 体空间的变化也暗含着 Lewis 碱性(即基团的推电子性) 的变化. 如一级烷基 $(45)$ 、二级烷基 $(46,47,50 \sim 52)$ 、三
级烷基(48 和 49)(图 11)分别与 $\mathrm{Cr}(\mathrm{acac})_{3} / \mathrm{MMAO}-3 \mathrm{~A}$ 组 成的体系, 均可以获得很高选择性的 $1-\mathrm{C}_{6}$, 这是基于取 代基的空间位阻但不是碱性. 含配体 $\mathbf{5 0}$ 的体系给出 $1-\mathrm{C}_{6}$ 在 $\mathrm{C}_{6}$ 中的选择性达到 $97 \%$, 而碱性最强的配体 48 的体系给出 $1-\mathrm{C}_{6}$ 在 $\mathrm{C}_{6}$ 中的选择性为 $94 \%$. 另外, 通过比 较具有相似碱性而位阻不同的配体(9、46 和 52 均由二 级胺合成, 44 和 45 均由一级胺合成), 发现取代基空间

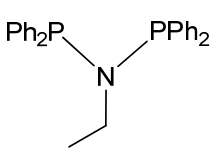

44

44/Cr(acac) $)_{3} / \mathrm{MMAO}-3 \mathrm{~A}$ methylcyclohexane $60^{\circ} \mathrm{C}, 4.5 \mathrm{MPa}$ Activity: $1.0 \times 10^{6} \mathrm{~g} /(\mathrm{g} \mathrm{Cr} \cdot \mathrm{h})$ Selectivity: (\%) $\mathrm{C}_{6}\left(1-\mathrm{C}_{6}\right): 17.5(40.7)$ $\mathrm{C}_{8}\left(1-\mathrm{C}_{8}\right): 63.0(97.3)$ $\mathrm{C}_{10+}: 14.6$ PE: 2.4

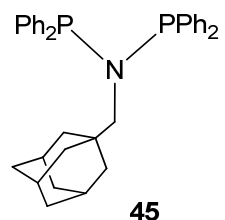

45

45/ $\mathrm{Cr}(\mathrm{acac})_{3} / \mathrm{MMAO}-3 \mathrm{~A} /$ methylcyclohexane $60^{\circ} \mathrm{C}, 4.5 \mathrm{MPa}$ Activity: $2.7 \times 10^{6} \mathrm{~g} /(\mathrm{g} \mathrm{Cr} \cdot \mathrm{h})$ Selectivity: (\%) $\mathrm{C}_{6}\left(1-\mathrm{C}_{6}\right): 20.6$ (71.6) $\mathrm{C}_{8}\left(1-\mathrm{C}_{8}\right): 67.3(99.1)$ $\mathrm{C}_{10+}: 10.3$ PE: 0.1

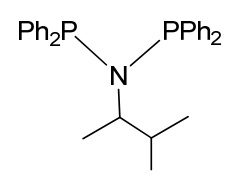

46

46/Cr(acac) 3 /MMAO-3A methylcyclohexane $60^{\circ} \mathrm{C}, 4.5 \mathrm{MPa}$

Activity: $1.9 \times 10^{6} \mathrm{~g} /(\mathrm{g} \mathrm{Cr} . \mathrm{h})$ Selectivity: (\%) $\mathrm{C}_{6}\left(1-\mathrm{C}_{6}\right): 24.3$ (85.1) $\mathrm{C}_{8}\left(1-\mathrm{C}_{8}\right): 65.7$ (99.5) $\mathrm{C}_{10+}: 8.8$ PE: 1.9

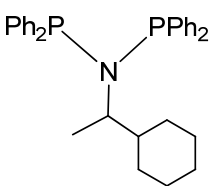

47

47/Cr(acac) $)_{3} / \mathrm{MMAO}-3 \mathrm{~A} /$ methylcyclohexane $60^{\circ} \mathrm{C}, 4.5 \mathrm{MPa}$<smiles>CC(C)(C)N(P)P</smiles>

48

48/Cr(acac) $)_{3} / \mathrm{MMAO}-3 \mathrm{~A}$ methylcyclohexane $60^{\circ} \mathrm{C}, 4.5 \mathrm{MPa}$

Activity: $0.32 \times 10^{6} \mathrm{~g} /(\mathrm{g} \mathrm{Cr} \cdot \mathrm{h})$ Activity: $1.6 \times 10^{6} \mathrm{~g} /(\mathrm{g} \mathrm{Cr} \cdot \mathrm{h})$

Selectivity: (\%) Selectivity: (\%)

$\mathrm{C}_{6}\left(1-\mathrm{C}_{6}\right): 25.1(86.2) \quad \mathrm{C}_{6}\left(1-\mathrm{C}_{6}\right): 32.5(94.0)$

$\mathrm{C}_{8}\left(1-\mathrm{C}_{8}\right): 65.5(99.4) \quad \mathrm{C}_{8}\left(1-\mathrm{C}_{8}\right): 58.0(99.5)$

$\mathrm{C}_{10+}: 8.4$

PE: 0.6

$\mathrm{PE} \cdot 12$

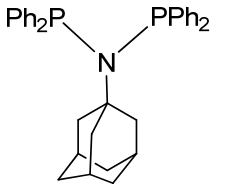

49

49/Cr(acac) $)_{3} / \mathrm{MMAO}-3 \mathrm{~A}$ methylcyclohexane $60^{\circ} \mathrm{C}, 4.5 \mathrm{MPa}$ Activity: $2.6 \times 10^{6} \mathrm{~g} /(\mathrm{g} \mathrm{Cr} \cdot \mathrm{h})$ Selectivity: (\%) $\mathrm{C}_{6}\left(1-\mathrm{C}_{6}\right): 30.8(94.5)$ $\mathrm{C}_{8}\left(1-\mathrm{C}_{8}\right): 59.3$ (99.6) $\mathrm{C}_{10+}: 8.5$

PE: 1.5<smiles>C[Si](C)(P)N(P)P</smiles>

53

53/Cr(acac) $)_{3} / \mathrm{MMAO}-3 \mathrm{~A}$ methylcyclohexane $60^{\circ} \mathrm{C}, 4.5 \mathrm{MPa}$ Activity: $2.0 \times 10^{4} \mathrm{~g} /(\mathrm{g} \mathrm{Cr} \cdot \mathrm{h})$ Selectivity: (\%) $\mathrm{C}_{6}\left(1-\mathrm{C}_{6}\right): 16.9(45.0)$ $\mathrm{C}_{8}\left(1-\mathrm{C}_{8}\right): 47.3(95.7)$ $\mathrm{C}_{10+}: 20.9$ PE: 12.9

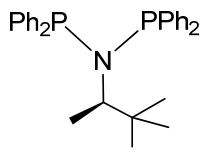

50

50/Cr(acac) $)_{3} / \mathrm{MMAO}-3 \mathrm{~A}$ methylcyclohexane $60{ }^{\circ} \mathrm{C}, 4.5 \mathrm{MPa}$ Activity: $1.5 \times 10^{6} \mathrm{~g} /(\mathrm{g} \mathrm{Cr} \cdot \mathrm{h})$ Selectivity: (\%) $\mathrm{C}_{6}\left(1-\mathrm{C}_{6}\right): 44.9$ (97.4) $\mathrm{C}_{8}\left(1-\mathrm{C}_{8}\right): 44.2(99.4)$ $\mathrm{C}_{10+}: 9.3$ PE: 1.5<smiles>Pc1ccccc1</smiles>
54

54/Cr(acac) $)_{3} / \mathrm{MMAO}-3 \mathrm{~A}$ methylcyclohexane $60^{\circ} \mathrm{C}, 4.5 \mathrm{MPa}$ Activity: $6.6 \times 10^{5} \mathrm{~g} /(\mathrm{g} \mathrm{Cr} \cdot \mathrm{h})$ Selectivity: (\%) $\mathrm{C}_{6}\left(1-\mathrm{C}_{6}\right): 18.3(39.2)$ $\mathrm{C}_{8}\left(1-\mathrm{C}_{8}\right): 56.6$ (99.6) $\mathrm{C}_{10+}: 16.2$ PE: 6.3<smiles>C[C@H](N(P)Pc1ccccc1)C(C)(C)C</smiles>

51

51/Cr(acac) 3 /MMAO-3A methylcyclohexane $60^{\circ} \mathrm{C}, 4.5 \mathrm{MPa}$

Activity: $1.4 \times 10^{6} \mathrm{~g} /(\mathrm{g} \mathrm{Cr} \cdot \mathrm{h})$ Selectivity: (\%) $\mathrm{C}_{8}\left(1-\mathrm{C}_{8}\right): 45.6(99.4)$ $\mathrm{C}_{10+}: 9.5$

PE: 1.5
$\mathrm{C}_{6}\left(1-\mathrm{C}_{6}\right): 43.5$ (97.2)<smiles>CC(N(P)P)N(P)C(C)(C)C</smiles>

52

52/Cr(acac) $)_{3} / \mathrm{MMAO}-3 \mathrm{~A} /$ methylcyclohexane $60^{\circ} \mathrm{C}, 4.5 \mathrm{MPa}$ Activity: $1.4 \times 10^{6} \mathrm{~g} /(\mathrm{g} \mathrm{Cr}-\mathrm{h})$ $\mathrm{C}_{6}\left(1-\mathrm{C}_{6}\right): 43.3(97.2)$ $\mathrm{C}_{8}\left(1-\mathrm{C}_{8}\right): 46.1(99.4)$

$\mathrm{C}_{10+}: 9.4$

PE: 1.2<smiles>Pc1ccccc1</smiles>

55
55/Cr(acac $)_{3} / \mathrm{MMAO}-3 \mathrm{~A} /$ methylcyclohexane $60^{\circ} \mathrm{C}, 4.5 \mathrm{MPa}$ Activity: $1.8 \times 10^{6} \mathrm{~g} /(\mathrm{g} \mathrm{Cr} \cdot \mathrm{h})$ Selectivity: (\%) $\mathrm{C}_{6}\left(1-\mathrm{C}_{6}\right): 17.6$ (75.5) $\mathrm{C}_{8}\left(1-\mathrm{C}_{8}\right): 69.4$ (99.0) $\mathrm{C}_{10+:}: 10.9$ PE: 1.4

图 11 [PNP]配体 $\mathbf{4 4} \sim 55$ 的结构式以及催化结果

Figure 11 Schematic structures of [PNP] ligands $44 \sim 55$ and catalytic results 
效应明显比碱性的影响要重要.

随着氮原子上取代基空间位阻的增大， $\alpha$-烯烃的总 选择性有所提高, 而 $\mathrm{C}_{6}$ 比例增加的趋势较 $\mathrm{C}_{8}$ 比例减少 的趋势大. 原因可能是氮原子上取代基尺寸的增大引起 磷原子上的苯环之间的排斥作用变强, 从而间接地影响 到金属碳环中间体的稳定性 ${ }^{[65]}$.

配体 50 52 含 $R 、 S$ 以及外消旋的 3,3-二甲基-2丁基, 与 $\mathrm{Cr}(\mathrm{acac})_{3} / \mathrm{MMAO}-3 \mathrm{~A}$ 组成的催化剂给出相似 的催化结果, 说明取代基的旋光异构性对产物的影响不 大. 再考察氮原子上的杂原子取代基如三甲基硅基(配 体 53)的影响, 结果表明 $\alpha$-烯烃的选择性达到 $53 \%$, 但 是聚合物相对较高 $(13 \%)$, 且催化活性很低 $\left[2.0 \times 10^{4} \mathrm{~g} /(\mathrm{g}\right.$ $\mathrm{Cr} \bullet \mathrm{h})$ ]. 原因可能是配体 53 在 MMAO-3A 存在下不稳定. 但是, 采用相应的金属配合物进行催化测试, 反应速率 提高到 $2.0 \times 10^{6} \mathrm{~g} /(\mathrm{g} \mathrm{Cr} \bullet \mathrm{h}), \alpha$-烯烃的总选择性也升到 $75 \%$. 尽管氮硅键不够稳定，但是相应的铬配合物却可 以稳定存在. 因此, 原位混合用于催化时形成较多的聚 合物且选择性很低的原因应归处于配体 53 的分解形成 了配体 36.

[PNP] 配体 54 和 55 含双位官能基, 在催化过程中自 然会形成双位活性中心, 但是催化活性和选择性分别与 单位的 44 和 8 的相当.

2007 年, Killian 等 ${ }^{[70]}$ 系统地研究了氮原子上芳基取 代基的结构和尺寸对 $[\mathrm{PNP}] / \mathrm{Cr}$ (acac) $3 / \mathrm{MMAO}-3 \mathrm{~A}$ 催化体 系的影响. 研究发现, 增加氮原子上芳基邻位基团的空 间位阻, 可以提高 1-辛烯的选择性至 56\%; 而在氮原子 和芳基之间引入一个碳原子, 同时再在这个碳原子上引 入一个支链, 可以获得高达 64\%的 1-辛烯选择性, 其催 化活性也超过 $1.0 \times 10^{6} \mathrm{~g} /(\mathrm{g} \mathrm{Cr} \bullet \mathrm{h})$.

对比配体 10, 56, 57, 58 我们可以清楚地看到, 增大 苯基邻位取代基的体积 (从 $\mathrm{H}$ 到异丙基), $\mathrm{C}_{6}$ 和 $1-\mathrm{C}_{6}$ 的选 择性都有所提高 $\left(1-\mathrm{C}_{6}\right.$ 在 $\mathrm{C}_{6}$ 中的选择性分别是 $67.8 \%$, $56.6 \%, 67.2 \%, 86.2 \%$ ), 而相应的 $\mathrm{C}_{6}$-环状化合物含量明 显降低, 且 $\mathrm{C}_{8}$ 选择性也降低. 这些结果表明配体空间位 阻的改变导致了产物选择性的改变. 值得注意的是, 空 间位阻最大的配体 $\mathbf{5 8}$ 得到最低的催化活性, 其中 PE 的 生成量最多. 在芳基的 2 位和 6 位引入甲基(配体 59), $\alpha$ 烯烃的总选择性较配体 58 的提高了 $1.5 \%$. 与配体 56 比 较, $1-\mathrm{C}_{6}$ 选择性提高了 $28.8 \%, \alpha$-烯烃的总选择性提高 $12.5 \%$.

从 Bollmann 等 ${ }^{[20]}$ 的研究结果可以看到, 当氮原子 上为烷基取代基时， $\alpha$-分支效应使得 $\alpha$-烯烃的总选择性 有所提高. 因此, 在本系列配体中的 $\mathrm{N}$ 原子和芳基之间 引入碳原子(图 12), 能同时兼顾 $\alpha$ 分支效应和芳基的影 响. 如引入亚甲基(配体 41), 降低了空间位阻, 催化剂
的活性提高了 $30 \%$, 但产物的组成变化不大. 进一步在 亚甲基上引入甲基而重新增大空间位阻(配体 60), $\alpha$-烯 烃的总选择性较配体 41 的提高了 $13.8 \%$, 而 $\mathrm{C}_{6}$ 中环状 产物降低了 $33.6 \%$. 基于配体 60 的催化剂给出最高的 $\mathrm{C}_{8}$ 选择性(64.1\%)和最高的 $\alpha$-烯烃总选择性(84.3\%). 含 配体 6 和 7 的催化剂也具有高的催化活性 $\left(>1.0 \times 10^{6}\right.$ $\mathrm{g} /(\mathrm{g} \mathrm{Cr} \bullet \mathrm{h}))$ 和低的 $\mathrm{PE}$ 产物分率 $(0.6 \%)$. 为继续增大配体 的空间位阻，可以在亚甲基上引入苯基(配体 61), 但是 相应的催化剂的 $\alpha$-烯烃总选择性没有提高, PE产物反而 提高了很多 $(25.8 \%$ ), 催化活性也较 60 的降低了 $40 \%$. 这些结果难以从上述的结构特性来解释，可能与苄基位 上的质子酸性有关. 当在亚甲基上引入苠基(配体 62), 催化选择性与配体 60 的相当, 可以看出这样的结果是 基于配体 60 和 62 的结构相似性.

在氮原子引入更大的荎基时(配体 63)， $\alpha$-烯烃的总 选择性提高很少 $(72.6 \%$ ). 同样，当在亚甲基上引入菜基 时(配体 64), $\mathrm{C}_{6}$ 和 1- $\mathrm{C}_{6}$ 的选择性保持相对不变, $\mathrm{C}_{8}$ 产物 的选择性降低了 $7.1 \%$, 催化活性则提高了将近 $40 \%$, 达 到 $7.0 \times 10^{5} \mathrm{~g} /(\mathrm{g} \mathrm{Cr} \bullet \mathrm{h})$, 与配体 10, 56 59, 41 组成的催 化体系的催化结果不同. 在配体 64 的基础上引入 $\alpha$ 分支 得到配体 65, $\alpha$-烯烃的总选择性能显著提高到 $84.5 \%$, 其中 $1-\mathrm{C}_{6}$ 的选择性提高了 $20.5 \%$, 活性超过 $1.0 \times 10^{6}$ $\mathrm{g} /(\mathrm{g} \mathrm{Cr} \bullet \mathrm{h})$.

总体而言, $\mathrm{P}$ 上含芳基取代基的 $[\mathrm{PNP}]$ 配体的选择性 较含环己基的催化选择性低. 除了配体的空间位阻效应 外, 相应的电子效应也不容忽视. 为此, 他们在 $\mathrm{N}$ 上芳 基的对位引入给电子性的叔丁基(66)和甲氧基(68)以及 硝基(67), 以考察电子效应的影响. 含给电子性的 66 的 催化剂的活性更高, 且 PE 产物少; 而含吸电子性的 67 的 $\alpha$-烯烃总选择性降低了 $9.4 \%, \mathrm{PE}$ 产物却提高了 16 . $2 \%$. 对于含配体 68 的催化剂, 催化活性较配体 10 的低 了近 $50 \%, 1-\mathrm{C}_{6}$ 的选择性降低了 $40.2 \%, \alpha$-烯烃的总选择 性降低了 3. $9 \%$. 推测极有可能是甲氧基和铝氧烷助剂 之间发生了作用. 因此，上述结果说明 $\mathrm{N}$ 上芳基的电子 效应确实会对选择性有一定的影响.

2007 年, 姜涛等 ${ }^{[40]}$ 对 9/Cr(acac) 3 在不同助剂作用下 催化性能进行了研究. 结果显示, MMAO、MAO 和 EAO 是有效的助催化剂, 其中 $9 / \mathrm{Cr}(\mathrm{acac})_{3} / \mathrm{MMAO}$ 的催化体 系得到 $7.6 \times 10^{5} \mathrm{~g} /(\mathrm{g} \mathrm{Cr} \cdot \mathrm{h})$ 的催化活性, 1-辛烯的选择性 达到 $71.3 \%$. 使用 $i$-BAO 或 EAO, 亚甲基环戊烷的比例 明显降低。

同年, McGuinness 等 ${ }^{[71]}$ 基于 $\mathbf{9} / \mathrm{CrCl}_{3}(\mathrm{THF})_{3} / \mathrm{AlEt}_{3}$ 体 系, 加入第二助剂来考察其对催化活性、催化剂稳定性 和 1 - 辛烯选择性的影响. 第二助剂包括 $\mathrm{B}\left(\mathrm{C}_{6} \mathrm{~F}_{5}\right)_{3}$, $\mathrm{Al}\left(\mathrm{OC}_{6} \mathrm{~F}_{5}\right)_{3},\left[\left(\mathrm{Et}_{2} \mathrm{O}\right)_{2} \mathrm{H}\right]\left[\mathrm{Al}\left(\mathrm{OC}_{6} \mathrm{~F}_{5}\right)_{4}\right], \quad\left[\mathrm{Ph}_{3} \mathrm{C}\right]\left[\mathrm{Ta}\left(\mathrm{OC}_{6} \mathrm{~F}_{5}\right)_{6}\right]$, 


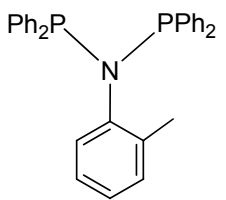

56

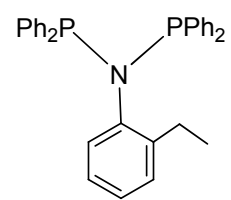

57

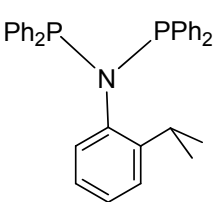

58<smiles>Cc1cccc(C)c1N(P)Pc1ccccc1</smiles>

59<smiles>CC(c1ccccc1)N(P)Pc1ccccc1</smiles>

60
56/Cr(acac) $)_{3} / \mathrm{MMAO}-3 \mathrm{~A}$ methylcyclohexane $60{ }^{\circ} \mathrm{C}, 5.0 \mathrm{MPa}$

Activity: $5.3 \times 10^{5} \mathrm{~g} /(\mathrm{g} \mathrm{Cr} \cdot \mathrm{h})$

Selectivity: (\%)

$\mathrm{C}_{6}\left(1-\mathrm{C}_{6}\right): 27.1$ (56.6)

$\mathrm{C}_{8}\left(1-\mathrm{C}_{8}\right): 56.8(97.0)$

$\mathrm{C}_{10+}: 9.9$

PE: 4.8
57/Cr(acac) ${ }_{3} / \mathrm{MMAO}-3 \mathrm{~A} /$ methylcyclohexane $60^{\circ} \mathrm{C}, 5.0 \mathrm{MPa}$

Activity: $5.6 \times 10^{5} \mathrm{~g} /(\mathrm{g} \mathrm{Cr} \cdot \mathrm{h})$

Selectivity: (\%)

$\mathrm{C}_{6}\left(1-\mathrm{C}_{6}\right): 29.4$ (67.2)

$\mathrm{C}_{8}\left(1-\mathrm{C}_{8}\right): 57.0(97.8)$

$\mathrm{C}_{10+}: 9.6$

PE: 3.0
58/Cr(acac) $)_{3} / \mathrm{MMAO}-3 \mathrm{~A} /$ methylcyclohexane $60{ }^{\circ} \mathrm{C}, 5.0 \mathrm{MPa}$

Activity: $1.6 \times 10^{5} \mathrm{~g} /(\mathrm{g} \mathrm{Cr} \cdot \mathrm{h})$

Selectivity: (\%)

$\mathrm{C}_{6}\left(1-\mathrm{C}_{6}\right): 33.4(86.2)$

$\mathrm{C}_{8}\left(1-\mathrm{C}_{8}\right): 52.9(99.2)$

$\mathrm{C}_{10+}: 6.0$

PE: 7.8
59/Cr(acac) ${ }_{3} / \mathrm{MMAO}-3 \mathrm{~A} /$ methylcyclohexane $60{ }^{\circ} \mathrm{C}, 5.0 \mathrm{MPa}$

Activity: $5.0 \times 10^{5} \mathrm{~g} /(\mathrm{g} \mathrm{Cr} \cdot \mathrm{h})$ Selectivity: (\%)

$\mathrm{C}_{6}\left(1-\mathrm{C}_{6}\right): 31.0(85.4)$

$\mathrm{C}_{8}\left(1-\mathrm{C}_{8}\right): 56.9(99.0)$

$\mathrm{C}_{10+}: 7.5$

PE: 3.8

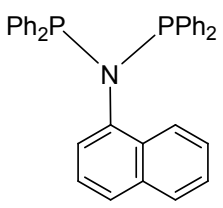

63/Cr(acac) $)_{3} / \mathrm{MMAO}-3 \mathrm{~A}$ methylcyclohexane $60{ }^{\circ} \mathrm{C}, 5.0 \mathrm{MPa}$

Activity: $5.2 \times 10^{5} \mathrm{~g} /(\mathrm{g} \mathrm{Cr} \cdot \mathrm{h})$ Selectivity: (\%)

$\mathrm{C}_{6}\left(1-\mathrm{C}_{6}\right): 25.5$ (63.9)

$\mathrm{C}_{8}\left(1-\mathrm{C}_{8}\right): 57.9(97.3)$

$\mathrm{C}_{10+}: 11.9$

PE: 3.4<smiles>O=[N+]([O-])c1ccc(N(Pc2ccccc2)Pc2ccccc2)cc1</smiles>

67

67/Cr(acac) $)_{3} / \mathrm{MMAO}-3 \mathrm{~A} /$ methylcyclohexane $60{ }^{\circ} \mathrm{C}, 5.0 \mathrm{MPa}$

Activity: $9.3 \times 10^{5} \mathrm{~g} /(\mathrm{g} \mathrm{Cr} \cdot \mathrm{h})$

Selectivity: (\%)

$\mathrm{C}_{6}\left(1-\mathrm{C}_{6}\right): 14.8$ (53.5)

$\mathrm{C}_{8}\left(1-\mathrm{C}_{8}\right): 53.3$ (96.9)

$\mathrm{C}_{10+}: 16.9$

PE: 16.2

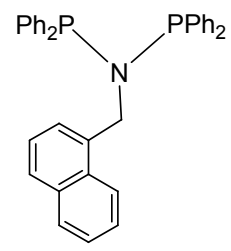

64
$60 / \mathrm{Cr}(\mathrm{acac})_{3} / \mathrm{MMAO}-3 \mathrm{~A} /$ methylcyclohexane $60^{\circ} \mathrm{C}, 5.0 \mathrm{MPa}$

Activity: $1.0 \times 10^{6} \mathrm{~g} /(\mathrm{g} \mathrm{Cr} . \mathrm{h})$ Selectivity: (\%) $\mathrm{C}_{6}\left(1-\mathrm{C}_{6}\right): 27.0$ (77.0) $\mathrm{C}_{8}\left(1-\mathrm{C}_{8}\right): 64.1$ (99.1)

$\mathrm{C}_{10+}: 7.1$

PE: 0.6
65/Cr(acac) $)_{3} / \mathrm{MMAO}-3 \mathrm{~A}$ $60{ }^{\circ} \mathrm{C}, 5.0 \mathrm{MPa}$ Activity: $1.0 \times 10^{6} \mathrm{~g} /(\mathrm{g} \mathrm{Cr} \cdot \mathrm{h})$ $\mathrm{C}_{6}\left(1-\mathrm{C}_{6}\right): 27.1(82.1)$ $\mathrm{C}_{8}\left(1-\mathrm{C}_{8}\right): 62.8(99.2)$

$\mathrm{C}_{10+}: 8.9$

PE: 0.8 methylcyclohexane $60{ }^{\circ} \mathrm{C}, 5.0 \mathrm{MPa}$

Selectivity: (\%)

$\mathrm{C}_{6}\left(1-\mathrm{C}_{6}\right): 17.7(53.5)$

$\mathrm{C}_{8}\left(1-\mathrm{C}_{8}\right): 62.3(96.6)$

$\mathrm{C}_{10+}: 16.4$

PE: 1.9
64/Cr(acac) $)_{3} / \mathrm{MMAO}-3 \mathrm{~A} /$ methylcyclohexane $60{ }^{\circ} \mathrm{C}, 5.0 \mathrm{MPa}$

Activity: $7.1 \times 10^{5} \mathrm{~g} /(\mathrm{g} \mathrm{Cr} \cdot \mathrm{h})$

Selectivity: (\%)

$\mathrm{C}_{6}\left(1-\mathrm{C}_{6}\right): 22.4$ (61.6)

$\mathrm{C}_{8}\left(1-\mathrm{C}_{8}\right): 65.0$ (98.4)

$\mathrm{C}_{10+}: 10.4$

PE: 1.0

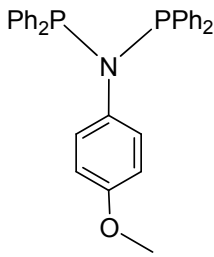

68/Cr(acac) $)_{3} / \mathrm{MMAO}-3 \mathrm{~A} /$

methylcyclohexane

$60^{\circ} \mathrm{C}, 5.0 \mathrm{MPa}$

Activity: $3.9 \times 10^{5} \mathrm{~g} /(\mathrm{g} \mathrm{Cr} \cdot \mathrm{h})$

Selectivity: (\%)

$\mathrm{C}_{6}\left(1-\mathrm{C}_{6}\right): 22.1$ (40.2)

$\mathrm{C}_{8}\left(1-\mathrm{C}_{8}\right): 58.2(96.7)$

$\mathrm{C}_{10+}: 13.8$

PE: 4.6

图 12 [PNP]配体 56 68 的结构式以及催化结果

Figure 12 Schematic structures of [PNP] ligands $56 \sim 68$ and catalytic results

$\left(\mathrm{Et}_{2} \mathrm{O}\right) \mathrm{Al}\left\{\mathrm{OCH}\left(\mathrm{C}_{6} \mathrm{~F}_{5}\right)_{2}\right\}_{3}, \quad\left(\mathrm{Et}_{2} \mathrm{O}\right) \mathrm{Al}\left\{\mathrm{OC}\left(\mathrm{CF}_{3}\right)_{3}\right\}_{3}$, $\left[\mathrm{Ph}_{3} \mathrm{C}\right]\left[\mathrm{Al}\left\{\mathrm{OC}\left(\mathrm{CF}_{3}\right)_{3}\right\}_{4}\right], \quad\left[\mathrm{Ph}_{3} \mathrm{C}\right]\left[\mathrm{A} 1 \mathrm{~F}\left\{\mathrm{OC}\left(\mathrm{CF}_{3}\right)_{3}\right\}_{3}\right]$, $\left[\mathrm{Ph}_{3} \mathrm{C}\right]\left[\left\{\left(\mathrm{F}_{3} \mathrm{C}\right)_{3} \mathrm{CO}\right\}_{3} \mathrm{Al}-\mathrm{F}-\mathrm{Al}\left\{\mathrm{OC}\left(\mathrm{CF}_{3}\right)_{3}\right\}_{3}\right],\left[\mathrm{Ph}_{3} \mathrm{C}\right][\mathrm{C}-$ $\mathrm{B}_{11} \mathrm{H}_{6} \mathrm{Br}_{6}$ ], 不同第二助剂导致 1-辛烯的选择性从 $5 \%$ 到
$72 \%$ 变化，这可能与助剂同上述催化体系的反应程度有 关，这点我们在前面的助剂以及溶剂部分讨论过.

随后，Wasserscheid 等 ${ }^{[43]}$ 报道了一系列氮原子上键 连环烷基取代基的 PNP 配体 $(\mathbf{8}, 27,28,69 \sim 80$ )(图 13)与 


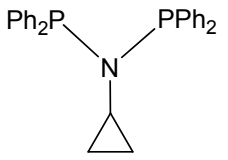

69
69/Cr(acac) $)_{3} / \mathrm{MMAO}-3 \mathrm{~A} /$ methylcyclohexane $60^{\circ} \mathrm{C}, 4.5 \mathrm{MPa}$ Activity: $4.6 \times 10^{5} \mathrm{~g} /(\mathrm{g}$ Cr.h) Selectivity: (\%) $\mathrm{C}_{6}\left(1-\mathrm{C}_{6}\right): 19.5(44.3)$ $\mathrm{C}_{8}\left(1-\mathrm{C}_{8}\right): 62.5$ (96.5) $\mathrm{C}_{10+}: 13.7$ PE: 1.8

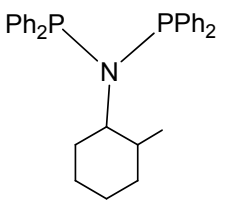

73

73/Cr(acac) $)_{3} / \mathrm{MMAO}-3 \mathrm{~A} /$ cyclohexane $60^{\circ} \mathrm{C}, 4.5 \mathrm{MPa}$ Activity: $2.3 \times 10^{6} \mathrm{~g} /(\mathrm{g} \mathrm{Cr} . \mathrm{h})$ Selectivity: (\%) $\mathrm{C}_{6}\left(1-\mathrm{C}_{6}\right): 26.6(85.4)$ $\mathrm{C}_{8}\left(1-\mathrm{C}_{8}\right): 63.7$ (99.5) $\mathrm{C}_{10+}$ : ndr PE: 0.7

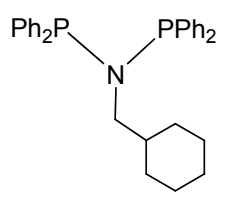

77

77/Cr(acac) $)_{3}$ MMAO-3A/ methylcyclohexane $60^{\circ} \mathrm{C}, 4.5 \mathrm{MPa}$ Activity: $4.8 \times 105 \mathrm{~g} /(\mathrm{g}$ Cr.h) Selectivity: (\%) $\mathrm{C}_{6}\left(1-\mathrm{C}_{6}\right): 19.8(45.8)$ $\mathrm{C}_{8}\left(1-\mathrm{C}_{8}\right): 63.9(97.3)$ $\mathrm{C}_{10+}: \mathrm{ndr}$ PE: 1.6

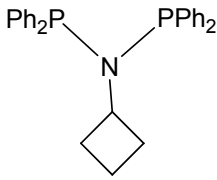

70

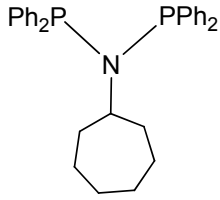

71

70/Cr(acac) $)_{3} / \mathrm{MMAO}-3 \mathrm{~A} /$ methylcyclohexane $60^{\circ} \mathrm{C}, 4.5 \mathrm{MPa}$

Activity: $3.9 \times 10^{5} \mathrm{~g} /(\mathrm{g} \mathrm{Cr} . \mathrm{h})$ Selectivity: (\%)

$\mathrm{C}_{6}\left(1-\mathrm{C}_{6}\right): 19.2(47.8)$

$\mathrm{C}_{8}\left(1-\mathrm{C}_{8}\right): 61.2(97.0)$

$C_{10}: 16.0$

PE: 1.3

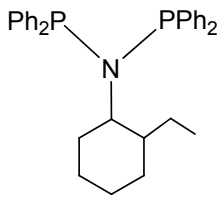

74/Cr(acac) $)_{3} / \mathrm{MMAO}-3 \mathrm{~A} /$ cyclohexane $60^{\circ} \mathrm{C}, 4.5 \mathrm{MPa}$ Activity: $2.2 \times 10^{6} \mathrm{~g} /(\mathrm{g}$ Cr.h $)$ Selectivity: (\%)

$\mathrm{C}_{6}\left(1-\mathrm{C}_{6}\right): 26.5$ (88.3)

$\mathrm{C}_{8}\left(1-\mathrm{C}_{8}\right): 64.5$ (99.6)

$\mathrm{C}_{10+}: \mathrm{ndr}$

PE: 0.3

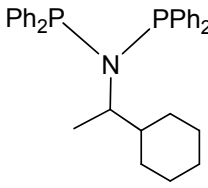

78

78/Cr(acac) $)_{3} / \mathrm{MMAO}-3 \mathrm{~A} /$ methylcyclohexane $60{ }^{\circ} \mathrm{C}, 4.5 \mathrm{MPa}$ Activity: $1.7 \times 10^{5} \mathrm{~g} /(\mathrm{g}$ Cr.h $)$ Selectivity: (\%) $\mathrm{C}_{6}\left(1-\mathrm{C}_{6}\right): 25.4$ (86.5) $\mathrm{C}_{8}\left(1-\mathrm{C}_{8}\right): 63.3$ (99.4) $\mathrm{C}_{10+}: \mathrm{ndr}$ PE: 1.9

71/Cr(acac) ${ }_{3} / \mathrm{MMAO}-3 \mathrm{~A} /$ methylcyclohexane $60{ }^{\circ} \mathrm{C}, 4.5 \mathrm{MPa}$ Selectivity: (\%)

$\mathrm{C}_{6}\left(1-\mathrm{C}_{6}\right): 19.8(74.3)$

$\mathrm{C}_{8}\left(1-\mathrm{C}_{8}\right): 68.1(99.0)$

$C_{10+}: 10.1$

PE: 1.2

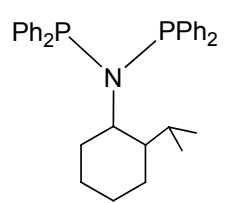

75

75/Cr(acac) 3 /MMAO-3A/ cyclohexane $60^{\circ} \mathrm{C}, 4.5 \mathrm{MPa}$

Selectivity: (\%)

$\mathrm{C}_{6}\left(1-\mathrm{C}_{6}\right): 29.6(92.9)$

$\mathrm{C}_{8}\left(1-\mathrm{C}_{8}\right): 59.8$ (99.6)

$\mathrm{C}_{10+}: \mathrm{ndr}$

PE: 1.3

79 methylcyclohexane $60{ }^{\circ} \mathrm{C}, 4.5 \mathrm{MPa}$

Selectivity: (\%)

$\mathrm{C}_{10+}: \mathrm{ndr}$

PE: 0.8

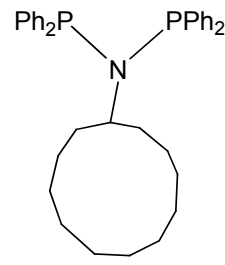

72

Activity: $7.4 \times 105 \mathrm{~g} /(\mathrm{g} \mathrm{Cr} . \mathrm{h})$

Activity: $1.9 \times 10^{6} \mathrm{~g} /(\mathrm{g}$ Cr.h)

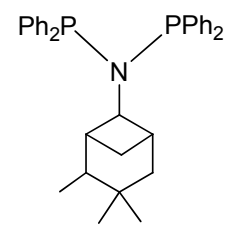

79/Cr(acac) 3 /MMAO-3A/

Activity: $6.1 \times 10^{5} \mathrm{~g} /(\mathrm{g}$ Cr.h)

$\mathrm{C}_{6}\left(1-\mathrm{C}_{6}\right): 27.1$ (89.1)

$\mathrm{C}_{8}\left(1-\mathrm{C}_{8}\right): 62.8$ (99.5)
72/Cr(acac) $)_{3} / \mathrm{MMAO}-3 \mathrm{~A} /$

methylcyclohexane $60^{\circ} \mathrm{C}, 4.5 \mathrm{MPa}$

Activity: $7.6 \times 10^{5} \mathrm{~g} /(\mathrm{g} \mathrm{Cr} . \mathrm{h})$

Selectivity: (\%)

$\mathrm{C}_{6}\left(1-\mathrm{C}_{6}\right): 22.6$ (84.6)

$\mathrm{C}_{8}\left(1-\mathrm{C}_{8}\right): 66.4$ (99.4)

$\mathrm{C}_{10+}: 9.6$

PE. 0.9

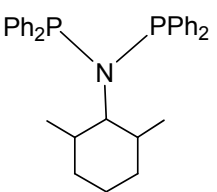

76

76/Cr(acac) $)_{3} / \mathrm{MMAO}-3 \mathrm{~A}$

cyclohexane

$60{ }^{\circ} \mathrm{C}, 4.5 \mathrm{MPa}$

Activity: $2.1 \times 10^{6} \mathrm{~g} /(\mathrm{g} \mathrm{Cr} . \mathrm{h})$

Selectivity: $(\%)$

$\mathrm{C}_{6}\left(1-\mathrm{C}_{6}\right): 43.0(95.4)$

$\mathrm{C}_{8}\left(1-\mathrm{C}_{8}\right): 48.7$ (99.7)

$\mathrm{C}_{10+}: \mathrm{ndr}$

PE: 0.3

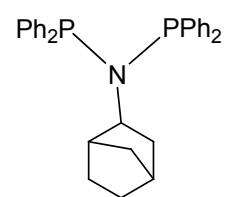

80

80/Cr(acac) $)_{3} / \mathrm{MMAO}-3 \mathrm{~A} /$ methylcyclohexane $60^{\circ} \mathrm{C}, 4.5 \mathrm{MPa}$ Activity: $8.5 \times 10^{5} \mathrm{~g} /(\mathrm{g}$ Cr.h) Selectivity: $(\%)$

$\mathrm{C}_{6}\left(1-\mathrm{C}_{6}\right) 22.8(70.5)$

$\mathrm{C}_{8}\left(1-\mathrm{C}_{8}\right): 65.3$ (98.8)

$\mathrm{C}_{10+}: \mathrm{ndr}$

PE: 3.0

图 $13[\mathrm{PNP}]$ 配体 69 $\sim 80$ 的结构式以及催化结果

Figure 13 Schematic structures of [PNP] ligands $69 \sim 80$ and catalytic results

$\mathrm{Cr}(\mathrm{acac})_{3}, \mathrm{MMAO}-3 \mathrm{~A}$ 组成催化体系的性能. 含 $\mathbf{8}$ 的催化 性能较好[活性为 $2.0 \times 10^{6} \mathrm{~g} /(\mathrm{g} \mathrm{Cr} \bullet \mathrm{h}), 1$ - 辛烯选择性为 68\%]. 氮原子上取代环烷基较小的配体所形成的催化 体系的 1- $\mathrm{C}_{6}$ 和 1- $\mathrm{C}_{8}$ 选择性很低, 其它副产物偏多. 然而 当环的大小由环丁基增长到环十二烷基时, 目标产物的 选择性显著提高. 当环烷基的碳数从 3 增加到 12 时, $\alpha-$ 烯烃的总选择性提高了 $16 \%$, 其中 $\mathrm{C}_{6}$ 中 $1-\mathrm{C}_{6}$ 的选择性
明显提高(从 $44 \%$ 到 $85 \%$ ), 副产物相应减少. 总体而言, 随着环烷基尺寸的增大，催化活性会相应地提高.

针对 $\mathrm{N}$ 上环己基的邻位取代基，增大取代基的位 阻， $\alpha$-烯烃的总选择性呈提高趋势。位阻越大，越有利 于 $1-\mathrm{C}_{6}$ 的形成. 含这些配体的催化体系是目前报道的活 性最高的催化剂之一，达到 $2.0 \times 10^{6} \mathrm{~g} /(\mathrm{g} \mathrm{Cr} \bullet \mathrm{h})$. 如在 $\mathrm{N}$ 上环己基的两个邻位各引入一个甲基(配体 76), $\mathrm{C}_{6}$ 中 
$1-\mathrm{C}_{6}$ 选择性达到 $95.4 \%$, 而且 $1-\mathrm{C}_{6}$ 和 $1-\mathrm{C}_{8}$ 的比例接近 $1 ： 1$. 当仅在环已基上的 2 位引入甲基(73)、乙基(74)、 异丙基(75)时, $\mathrm{C}_{8}$ 组分大于 $\mathrm{C}_{6}$ 组分.

将此配体引入 $\alpha$-分支结构基团，比较发现(配体 77 和 79), $\alpha$-烯烃的总选择性降低了 $9 \%$. 而将亚甲基上的 一个氢原子换作甲基(78), 空间位阻随之变大, $\alpha$-烯烃的 总选择性提高了 $13 \%$. 含配体 $\mathbf{8 0}$ 的催化剂选择性与含 $\mathbf{8}$ 的相似.

接着, 姜涛等 ${ }^{[44]}$ 研究了 $28 / \mathrm{Cr}(\mathrm{III}) / \mathrm{MAO} /$ 卤化物(二 氯甲烷、三氯甲烷、四氯甲烷、1,1,2-三氯乙烷、1,1,1三氯乙烷、1-溴丁烷、1-氯丁烷)四组分催化体系中卤化 物的作用. 结果表明, 二氯甲烷对 $1-\mathrm{C}_{8}$ 的催化活性和选 择性均优于三氯甲烷和四氯甲烷; 1,1,2-三氯乙烷对 $1-C_{8}$ 的选择性优于 $1,1,1-$ 三氯乙烷; 氯化物对 $1-C_{8}$ 的催 化活性和选择性均优于相应的溴化物. 因此, 卤化物的 空间位阻、分子稳定性、结构和类型对 $1-\mathrm{C}_{8}$ 的催化活性 和选择性都十分重要, 原因在前面阐述的助剂以及溶剂 部分中有讲到, 可能是卤化物与铬金属活性中心的相互 作用.

Hanton 等 ${ }^{[42]}$ 也在同时期研究了 [PNP]配体的羰基铬 化合物的催化性能 $\left([\mathbf{9}] \mathrm{Cr}(\mathrm{CO})_{4} 、\left\{[9] \mathrm{Cr}(\mathrm{CO})_{4}\right\}^{+}\left(\mathrm{BF}_{4}\right)^{-}\right.$、

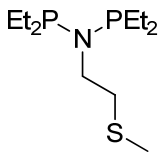

81

$81 / \mathrm{Cr}(\mathrm{acac})_{3} / \mathrm{MAO} /$ toluene $80^{\circ} \mathrm{C}, 3.0 \mathrm{MPa}$

Activity: $4.1 \times 10^{4} \mathrm{~g} /(\mathrm{g} \mathrm{Cr} \cdot \mathrm{h})$

Selectivity: (\%)

$\mathrm{C}_{6}\left(1-\mathrm{C}_{6}\right): 23.0$ (ndr)

$\mathrm{C}_{8}\left(1-\mathrm{C}_{8}\right): 43.9(97.7)$

$\mathrm{C}_{10+}: 22.9$

PE: 11.1

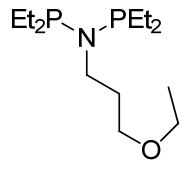

85

$85 / \mathrm{Cr}$ (acac) $)_{3} / \mathrm{MAO} /$ toluene $80^{\circ} \mathrm{C}, 3.0 \mathrm{MPa}$

Activity: $6.1 \times 10^{4} \mathrm{~g} /(\mathrm{g} \mathrm{Cr} \cdot \mathrm{h})$

Selectivity: (\%)

$\mathrm{C}_{6}\left(1-\mathrm{C}_{6}\right): 12.6$ (ndr)

$\mathrm{C}_{8}\left(1-\mathrm{C}_{8}\right): 25.8$ (97.8)

$\mathrm{C}_{10+}: 34.5$

PE: 26.9

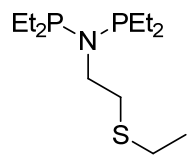

82

82/ $\mathrm{Cr}(\mathrm{acac})_{3} / \mathrm{MAO} /$ toluene $80^{\circ} \mathrm{C}, 3.0 \mathrm{MPa}$

Activity: $5.4 \times 10^{4} \mathrm{~g} /(\mathrm{g} \mathrm{Cr} \cdot \mathrm{h})$

Selectivity: (\%)

$\mathrm{C}_{6}\left(1-\mathrm{C}_{6}\right): 13.6$ (ndr)

$\mathrm{C}_{8}\left(1-\mathrm{C}_{8}\right): 27.9$ (98.5)

$\mathrm{C}_{10+}: 34.0$

PE: 24.3

$\left\{[9] \mathrm{Cr}(\mathrm{CO})_{4}\right\}^{+}\left(\mathrm{PF}_{6}\right)^{-}$. 以 $\mathrm{Et}_{3} \mathrm{Al}$ 为助催化剂, $\left[\mathrm{Al}\left(\mathrm{OC}\left(\mathrm{CF}_{3}\right)_{3}\right)_{4}\right]^{-}$为配位阴离子, 可有效催化乙烯四聚, 催化活性接近 $10^{5} \mathrm{~g} /(\mathrm{g} \mathrm{Cr} \bullet \mathrm{h}), 1$-辛烯选择性高达 $70 \%$. 翁 志强等 ${ }^{[72]}$ 则利用系列 PNP 配体 $81 \sim 87$ (图 14), 与 $\mathrm{CrCl}_{3}(\mathrm{THF})_{3} 、 \mathrm{MAO}$ 组成催化体系, 乙烯齐聚活性为 $10^{4}$ $\mathrm{g} /(\mathrm{g} \mathrm{Cr} \bullet \mathrm{h}), \mathrm{C}_{6}$ 选择性为 $9 \% \sim 27 \%, \mathrm{C}_{8}$ 选择性为 $19 \%$ $55 \%$.

2008 年，姜涛等 ${ }^{[73]}$ 报道了 $[\mathbf{8 8} \sim \mathbf{9 2}] / \mathrm{Cr}(\mathrm{acac})_{3} / \mathrm{MAO}$ 催化体系中 $[\mathrm{PNP}]$ 配体中氮原子上芳基取代基对催化活 性和 1-辛烯选择性的影响(图 15). 发现配体92 形成的催 化体系得到最高的 1-辛烯选择性 $71.0 \%$, 催化活性为 $10^{5} \mathrm{~g} /(\mathrm{g} \mathrm{Cr} \bullet \mathrm{h})$.

比较配体 56, 88, 89 发现, 随着芳基取代位置的变 化(从邻位到间位再到对位), 所形成的催化体系对 1-辛 烯的选择性逐渐升高(分别是 $55.1 \%$ ，66.5\%，69.3\%)，配 体 89 与苯基取代的配体 7 的选择性相当; 对于邻位取代 的配体 56, 57, 58, 90, 随着取代基位阻的增大，催化活 性和 1-辛烯的选择性均有降低的趋势; 而间位取代的配 体 88, 91, 92, 随着取代基位阻的增大，催化活性和 1-辛 烯的选择性均有升高的趋势.

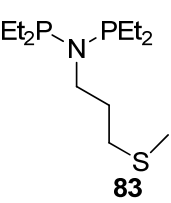

83/Cr(acac) $)_{3} / \mathrm{MAO} /$ toluene $80^{\circ} \mathrm{C}, 3.0 \mathrm{MPa}$ Activity: $4.4 \times 10^{4} \mathrm{~g} /(\mathrm{g} \mathrm{Cr} \cdot \mathrm{h})$ Selectivity: (\%) $\mathrm{C}_{6}\left(1-\mathrm{C}_{6}\right): 25.4$ (ndr) $\mathrm{C}_{8}\left(1-\mathrm{C}_{8}\right): 52.8$ (98.5) $\mathrm{C}_{10+}: 16.5$ PE: 4.9<smiles>CCPN(CC)CCc1ccccn1</smiles>

87

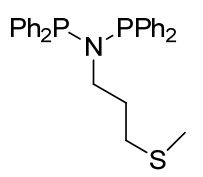

84

$84 / \mathrm{Cr}(\mathrm{acac})_{3} / \mathrm{MAO} /$ toluene $80^{\circ} \mathrm{C}, 3.0 \mathrm{MPa}$ Activity: $1.9 \times 10^{4} \mathrm{~g} /(\mathrm{g} \mathrm{Cr} \cdot \mathrm{h})$ Selectivity: (\%) $\mathrm{C}_{6}\left(1-\mathrm{C}_{6}\right): 3.5$ (ndr) $\mathrm{C}_{8}\left(1-\mathrm{C}_{8}\right): 12.5$ (97.0) $\mathrm{C}_{10+}: 21.3$ PE: 62.4

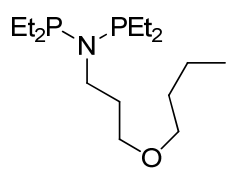

86

$86 / \mathrm{Cr}(\mathrm{acac})_{3} / \mathrm{MAO} /$ toluene $80{ }^{\circ} \mathrm{C}, 3.0 \mathrm{MPa}$ Activity: $4.9 \times 10^{4} \mathrm{~g} /(\mathrm{g} \mathrm{Cr} \cdot \mathrm{h})$ Selectivity: (\%) $\mathrm{C}_{6}\left(1-\mathrm{C}_{6}\right): 13.2$ (ndr) $\mathrm{C}_{8}\left(1-\mathrm{C}_{8}\right): 27.8$ (97.5) $\mathrm{C}_{10+}: 32.4$ PE: 26.4
87/Cr(acac) $3 / \mathrm{MAO} /$ toluene $80^{\circ} \mathrm{C}, 3.0 \mathrm{MPa}$

Activity: $3.1 \times 10^{4} \mathrm{~g} /(\mathrm{g} \mathrm{Cr} \cdot \mathrm{h})$ Selectivity: (\%)

$\mathrm{C}_{6}\left(1-\mathrm{C}_{6}\right): 9.1$ (ndr)

$\mathrm{C}_{8}\left(1-\mathrm{C}_{8}\right): 15.0(96.2)$

$\mathrm{C}_{10+}: 53.2$

PE: 23.4

图 14 [PNP]配体 81 87 的结构式以及催化结果

Figure 14 Schematic structures of [PNP] ligands $81 \sim 87$ and catalytic results 


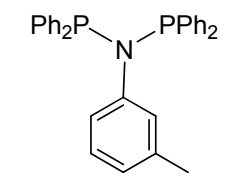

88

$88 / \mathrm{Cr}(\mathrm{acac})_{3} / \mathrm{MAO} /$ cyclohexane $60^{\circ} \mathrm{C}, 3.0 \mathrm{MPa}$ Activity: $3.5 \times 10^{4} \mathrm{~g} /\left(\mathrm{g}\right.$ Cr.h) Activity: $4.0 \times 10^{4} \mathrm{~g} /(\mathrm{g} \mathrm{Cr} \cdot \mathrm{h})$ Selectivity: (\%) $1-\mathrm{C}_{6}: 17.2$ $1-\mathrm{C}_{8}: 66.5$ $\mathrm{C}_{10+}: 1.7$ PE: 2.9

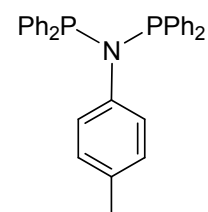

89 $89 / \mathrm{Cr}(\mathrm{acac})_{3} / \mathrm{MAO}$ cyclohexane $60^{\circ} \mathrm{C}, 3.0 \mathrm{MP}$

Selectivity: (\%)

$1-\mathrm{C}_{6}: 15.4$

$1-C_{8}: 69.3$

$\mathrm{C}_{10+}: 1.8$

PE: 2.1

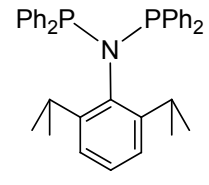

90

$90 / \mathrm{Cr}(\mathrm{acac})_{3} / \mathrm{MAO} /$ toluene $60{ }^{\circ} \mathrm{C}, 3.0 \mathrm{MPa}$ cyclohexane

$60^{\circ} \mathrm{C}, 3.0 \mathrm{MPa}$

Activity: $6.8 \times 10^{4} \mathrm{~g} /(\mathrm{g} \mathrm{Cr} \cdot \mathrm{h})$

Selectivity: (\%)

$1-\mathrm{C}_{6}: 14.3$

$1-\mathrm{C}_{8}: 77.2$

$\mathrm{C}_{10+}: 1.2$

PE: 3.1

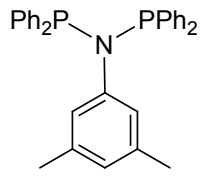

92

92/ $\mathrm{Cr}(\mathrm{acac})_{3} / \mathrm{MAO} /$

cyclohexane

$60{ }^{\circ} \mathrm{C}, 3.0 \mathrm{MPa}$

Activity: $9.6 \times 10^{4} \mathrm{~g} /(\mathrm{g} \mathrm{Cr} \cdot \mathrm{h})$

Selectivity: (\%)

$1-\mathrm{C}_{6}: 13.2$

$1-\mathrm{C}_{8}: 71.0$

$\mathrm{C}_{10+}: 0.6$

PE: 1.5

图 15 [PNP]配体 88 $\sim 92$ 的结构式以及催化结果

Figure 15 Schematic structures of [PNP] ligands $88 \sim 92$ and catalytic results

2012 年, 他们报道了配体 93 与 $\mathrm{Cr}(\mathrm{III}) 、 \mathrm{MAO}$ 组成 的催化体系(图 16)可以高活性高选择性地催化乙烯四 聚, 并考察了反应温度、摩尔比 $(\mathrm{Al} / \mathrm{Cr} 、 \mathrm{PNP} / \mathrm{Cr})$ 对催化 活性和 1-辛烯选择性的影响 ${ }^{[74]}$.<smiles>Pc1ccccc1</smiles>

93

93/Cr(III)/MAO/cyclohexane
$30{ }^{\circ} \mathrm{C}, 2.0 \mathrm{MPa}$
Activity: $2.3 \times 10^{4} \mathrm{~g} /(\mathrm{g} \mathrm{Cr}$ h)
Selectivity: $(\%)$
$1-\mathrm{C}_{6}: 24.2$
$1-\mathrm{C}_{8}: 61.8$
$\mathrm{C}_{10+}: 3.5$
$\mathrm{PE}: 0.9$

图 16 [PNP]配体 93 的结构式以及催化结果

Figure 16 The schematic structure of [PNP] ligand 93 and the catalytic result

实验结果显示, 随着温度的升高, 催化活性先升高 后降低, 在 $40{ }^{\circ} \mathrm{C}$ 时达到最大 $\left[5.0 \times 10^{4} \mathrm{~g} /(\mathrm{g} \mathrm{Cr} \bullet \mathrm{h})\right]$, 而 1辛烯的选择性逐渐降低, 由 $61.8 \%$ 降为 $40.7 \%$. 随着 $\mathrm{Al} / \mathrm{Cr}$ 比的增大, 催化活性也是呈先升高后降低趋势, 当 $\mathrm{Al} / \mathrm{Cr}$ 为 300 时最大 $\left[4.7 \times 10^{4} \mathrm{~g} /(\mathrm{g} \mathrm{Cr} \bullet \mathrm{h})\right], 1$-辛烯的选 择性从 $53.8 \%$ 升至 $57.2 \%$. 随压力的增大, 催化活性和 1-辛烯的选择性都相应地逐渐升高.

2013 年, Kim 等 ${ }^{[75]}$ 合成了一系列[PNP]配体 94 100 (图 17), 分别与 $\mathrm{CrCl}_{3}(\mathrm{THF})_{3}$ 或 $\mathrm{Cr}(\mathrm{acac})_{3} 、 \mathrm{MAO}$ 组 成催化体系, 考察了配体结构、温度对催化活性和选择 性的影响. 结果表明, 配体结构对催化活性和选择性的 影响很大. 含配体 94 的催化体系对乙烯齐聚没有选择
性; 含 100 的催化体系仅给出聚合产物; 含 95 97 催化 体系催化乙烯齐聚的活性在 $10^{4} \mathrm{~g} /(\mathrm{g} \mathrm{Cr} \bullet \mathrm{h})$ 数量级, $1-\mathrm{C}_{6}$ 的选择性为 $6 \% \sim 14 \%, 1-\mathrm{C}_{8}$ 选择性为 $43 \% \sim 62 \%$. 温度 对含 9 的催化活性和选择性影响较大, 对含 96 和 98 的 催化性能影响较小.

最近, 姜涛等 ${ }^{[76]}$ 研究了负载化的催化剂性能, 载体 (MAO 修饰的 HZSM-5, HY, NaY)不同，负载的方式不 同，催化乙烯四聚的活性和选择性也不同. ZSM-Cr 和 $\mathrm{HY}-\mathrm{Cr}$ 催化乙烯四聚的活性和选择性较低, 而 $\mathrm{NaY}-\mathrm{Cr}$ 催化乙烯四聚的活性和选择性较高(活性 $3.3 \times 10^{5} \mathrm{~g} /(\mathrm{g}$ $\mathrm{Cr} \cdot \mathrm{h}), 1$-辛烯选择性达 $66.1 \%$.

\section{7 总结与展望}

基于 $[\mathrm{PNP}]$ 骨架配体的铬基催化剂用于乙烯选择性 齐聚的研究自 2002 年起至今已开展了近 13 年. 该催化 体系开创了乙烯选择性四聚, 是铬系自 Phillips 三聚催 化剂后的又一重要的催化体系.

该催化剂体系显示了 [PNP]配位骨架的特点, 同时 $\mathrm{N}$ 和 $\mathrm{P}$ 上取代基在电子和立体空间的结构方面可以变 化. 这些结构特点在调控金属铬中心催化乙烯选择性齐 聚方面起到了关键的作用. 同时，反应体系的催化活性 又受到其它很多因素的影响, 如使用的助催化剂的种 类、溶剂、反应浓度、温度、压力、时间等. 主催化剂 和助催化剂的制备体现了丰富的合成化学，催化反应的 诸多因素的影响也展现了丰富的催化化学. 这些都构成 了 [PNP] 铬基催化剂催化乙烯选择性齐聚的新化学. 基 于这些特点, 我们相信该催化剂体系在将来的乙烯选择 性齐聚生产 1-辛烯方面具有发展潜力. 同时, 我们也注 意到该类催化剂的负载性研究, 但是不多. 这些研究将 为其工业化应用奠定基础. 因此, 负载型催化剂体系值 得化学工作者们去进一步深入地研究. 
<smiles>CN(C)N(Pc1ccccc1)c1ccccc1</smiles>

94

$94 / \mathrm{CrCl}_{3}(\mathrm{THF})_{3} / \mathrm{MAO} /$ toluene $60^{\circ} \mathrm{C}, 3.0 \mathrm{MPa}$ Activity: $1.4 \times 10^{4} \mathrm{~g} /(\mathrm{g} \mathrm{Cr} . \mathrm{h})$ Selectivity: (\%) $\mathrm{C}_{6}\left(1-\mathrm{C}_{6}\right): \mathrm{ndr}$ $\mathrm{C}_{8}\left(1-\mathrm{C}_{8}\right): \mathrm{ndr}$ $\mathrm{C}_{10+}: \mathrm{ndr}$ PE: 7.0

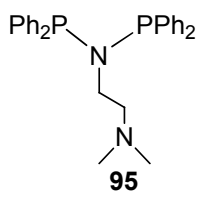

$95 / \mathrm{CrCl}_{3}(\mathrm{THF})_{3} / \mathrm{MAO} /$ toluene $60{ }^{\circ} \mathrm{C}, 3.0 \mathrm{MPa}$

Activity: $1.3 \times 10^{4} \mathrm{~g} /(\mathrm{g}$ Cr.h) Selectivity: (\%) $\mathrm{C}_{6}\left(1-\mathrm{C}_{6}\right): 23.3(62.2)$ $\mathrm{C}_{8}\left(1-\mathrm{C}_{8}\right): 46.3(93.7)$ $\mathrm{C}_{10+}: \mathrm{ndr}$ PE: 70.9<smiles>CC(C)N(CCN(Pc1ccccc1)c1ccccc1)C(C)C</smiles>

$96 / \mathrm{CrCl}_{3}(\mathrm{THF})_{3} / \mathrm{MAO} /$ toluene $60^{\circ} \mathrm{C}, 3.0 \mathrm{MPa}$ Activity: $1.5 \times 10^{4} \mathrm{~g} /(\mathrm{g}$ Cr.h $)$ Selectivity: (\%) $\mathrm{C}_{6}\left(1-\mathrm{C}_{6}\right): 23.7(51.5)$ $\mathrm{C}_{8}\left(1-\mathrm{C}_{8}\right): 64.8(96.0)$

$\mathrm{C}_{10+}: \mathrm{ndr}$ PE: 1.6<smiles>CCCCN(CCCC)CCN(Pc1ccccc1)c1ccccc1</smiles>

97

$97 / \mathrm{CrCl}_{3}(\mathrm{THF})_{3} / \mathrm{MAO} /$ toluene

$60{ }^{\circ} \mathrm{C}, 3.0 \mathrm{MPa}$

Activity: $1.4 \times 10^{4} \mathrm{~g} /(\mathrm{g}$ Cr.h $)$

Selectivity: (\%)

$\mathrm{C}_{6}\left(1-\mathrm{C}_{6}\right): 24.3(58.4)$

$\mathrm{C}_{8}\left(1-\mathrm{C}_{8}\right): 53.4(94.4)$

$\mathrm{C}_{10+}$ : ndr

PE: 48.8<smiles>CN(C)CCCN(Pc1ccccc1)Pc1ccccc1</smiles>

98

$98 / \mathrm{CrCl}_{3}(\mathrm{THF})_{3} / \mathrm{MAO} /$ toluene $60^{\circ} \mathrm{C}, 3.0 \mathrm{MPa}$ Activity: $1.1 \times 10^{4} \mathrm{~g} /(\mathrm{g}$ Cr.h $)$ Selectivity: (\%) $\mathrm{C}_{6}\left(1-\mathrm{C}_{6}\right): 21.9(61.2)$ $\mathrm{C}_{8}\left(1-\mathrm{C}_{8}\right): 64.6(94.0)$ $\mathrm{C}_{10+}: \mathrm{ndr}$ PE: 15.7

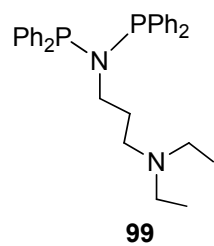

99/ $\mathrm{CrCl}_{3}(\mathrm{THF})_{3} / \mathrm{MAO} /$ toluene $60{ }^{\circ} \mathrm{C}, 3.0 \mathrm{MPa}$ Activity: $7.8 \times 10^{3} \mathrm{~g} /(\mathrm{g}$ Cr.h) Selectivity: (\%) $\mathrm{C}_{6}\left(1-\mathrm{C}_{6}\right): 26.6$ (55.6) $\mathrm{C}_{8}\left(1-\mathrm{C}_{8}\right): 62.4(95.2)$ $\mathrm{C}_{10+}: \mathrm{ndr}$ PE: 7.4<smiles>CN(C)CCCN(Pc1ccccc1)Pc1ccccc1</smiles>

$100 / \mathrm{CrCl}_{3}(\mathrm{THF})_{3} / \mathrm{MAO} /$ toluene

$60{ }^{\circ} \mathrm{C}, 3.0 \mathrm{MPa}$

Activity: $6.3 \times 10^{3} \mathrm{~g} /(\mathrm{g}$ Cr.h)

Selectivity: (\%)

$\mathrm{C}_{6}\left(1-\mathrm{C}_{6}\right)$ : ndr

$\mathrm{C}_{8}\left(1-\mathrm{C}_{8}\right): \mathrm{ndr}$

$\mathrm{C}_{10+}: \mathrm{ndr}$

PE: 96.3

图 17 [PNP]配体 94 100 的结构式以及催化结果

Figure 17 Schematic structures of [PNP] ligands $\mathbf{9 4} \sim \mathbf{1 0 0}$ and catalytic results

\section{References}

[1] Skupinska, J. Chem. Rev. 1991, 91, 613.

[2] Dixon, J. T.; Green, M. J.; Hess, F. M.; Morgan, D. H. J. Organomet. Chem. 2004, 689, 3641.

[3] Qian, B. Z. Petrochem. Ind. Technol. 2011, 18, 58 (in Chinese). (钱伯章, 石化技术, 2011, 18, 58.)

[4] McGuinness, D. S. Chem. Rev. 2011, 111, 2321.

[5] Agapie, T. Coord. Chem. Rev. 2011, 255, 861.

[6] Belov, G. P. Pet. Chem. 2012, 52, 139.

[7] (a) Jiang, T.; Chen, H. X.; Ning, Y. N.; Chen, W. Chin. Sci. Bull. 2006, 51, 391 (in Chinese).

(姜涛，陈洪侠，宁英男，陈伟，科学通报, 2006, 51, 391.)

(b) Song, X. F.; Ning, Y. N.; Jiang, T. Chem. Ind. Eng. Prog. 2008, 27, 693 (in Chinese).

(宋宪凤，宁英男，姜涛，化工进展，2008，27, 693.)

(c) Ning, Y. N.; Niu, B.; Jiang, T. Chem. Prod. Technol. 2009, 16, 19 (in Chinese)

(宁英男，牛博，姜涛，化工生产与技术, 2009, 16, 19.)

(d) Mao, G. L.; Ning, Y. N.; Niu, B.; Jiang, T. Chin. Sci. Bull. 2008, 53, 2592 (in Chinese).

(毛国梁，宁英男，牛博，姜涛，科学通报，2008，53，2592.)

(e) Zhao, T. Petrochem. Technol. Appl. 2010, 28, 439 (in Chinese). (赵檀，石化技术与应用, 2010, 28, 439.)

(f) Zhang, L. C.; Wu, H. F.; Li, J. W. China Synth. Resin Plast. 2010, 27, 68 (in Chinese).

(张立超，吴红飞，李建伟，合成树脂及塑料, 2010, 27, 68.)

[8] Lappin, G. Butene-1 and Other LLDPE Comonmers, Chem Sys- tems, Inc, New York, 1986

[9] Sauser, J. Alpha-olefins Applications Handbook, Marcel Dekker, New York, 1989.

[10] Guo, F.; Li, C. F.; Liu, J. W.; Chen, S. H.; Yang, A. W. Mod. Chem. Ind. 2010, 30, 92 (in Chinese).

(郭峰，李传峰，刘经伟，陈韶辉，杨爱武，现代化工，2010，30, 92.)

[11] (a) Small, B. L.; Brookhart, M.; Bennett, A. M. A. J. Am. Chem Soc. 1998, 120, 4049.

(b) Brookhart, B. L.; Small, M. J. Am. Chem. Soc. 1998, 120, 7143.

[12] Bennett. J. WO 98/27124, 1998 [Chem. Abstr. 1998, 129, 122973].

[13] (a) Britovsek, G. J. P.; Gibson, V. C.; Kimberley, B. S.; Maddox, P. J.; Williams, D. J. Chem. Commun. 1998, 849.

(b) Britovsek, G. J. P.; Bruse, M.; Gibson, V. C.; Kimberley, B. S.; Maddox, P. J.; Mastroianni, S.; McTavish, S. J.; Redshaw, C.; Solan, G. A.; Williams, D. J. J. Am. Chem. Soc. 1999, 121, 8728.

(c) Britovsek, G. J. P.; Mastroianni, S.; Solan, G. A.; Baugh, S. P. D.; Redshaw, C.; Gibson, V. C.; White, A. J. P.; Williams, D. J. Chem.-Eur. J. 2000, 6, 2221.

[14] Pillai, S. M.; Sivaram, S. Chem. Rev. 1986, 86, 353.

[15] Ziegler, K.; Martin, H. US 2943125, 1960 [Chem. Abstr. 1960, 54, 13467].

[16] Chauvin, Y. Angew. Chem., Int. Ed. 2006, 45, 3741.

[17] Robert, M. M.; Albans, S. US 3300458, 1967 [Chem. Abstr. 1967, $66,66008]$.

[18] Reagen, W. K. EP 0417477, 1991 [Chem. Abstr. 1991, 115, 93172].

[19] Carter, A.; Cohen, S. A.; Cooley, N. A.; Murphy, A.; Scutt, J.; Wass, D. F. Chem. Commun. 2002, 858 . 
[20] Bollmann, A.; Blann, K.; Dixon, J. T.; Hess, F. M.; Killian, E.; Maumela, H.; McGuinness, D. S.; Morgan, D. H.; Neveling, A.; Otto, S.; Overett, M.; Slawin, A. M. Z.; Wasserscheid, P.; Kuhlmann, S. J. Am. Chem. Soc. 2004, 126, 14712.

[21] Shaikh, Y.; Albahily, K.; Sutcliffe, M.; Fomitcheva, V.; Gambarotta, S.; Korobkov, I.; Duchateau, R. Angew. Chem., Int. Ed. 2012, 51, 1366.

[22] (a) Jefferson, R.; Nixon, J. F.; Painter, T. M.; Keat, R.; Stobbs, L. J. Chem. Soc., Dalton Trans. 1973, 1414.

(b) Payne, N. C.; Stephan, D. W. J. Organomet. Chem. 1981, 221, 203.

[23] (a) Wannagat, U.; Autzen, H. Z. Anorg. Allg. Chem. 1976, 420, 119. (b) Colquhoun, I. G.; McFarlane, W. J. Chem. Soc. Dalton Trans. $1977,1674$.

[24] (a) Chen, H. J.; Barendt, J. F.; Haltiwanger, R. C.; Hill, T. G.; Norman, A. D. Phosphorus Sulfur, Relat. Elem. 1986, 26, 155.

(b) Davies, A. R.; Dronsfield, A. T.; Haszeldine, R. N.; Taylor, D. R. J. Chem. Soc., Perkin Trans. 1 1973, 379.

(c) Jefferson, R.; Nixon, J. F.; Painter, T. M.; Keat, R.; Stobbs, L. J. Chem. Soc., Dalton Trans. 1973, 1414.

(d) Cross, R. J.; Green, T. H.; Keat, R. J. Chem. Soc., Dalton Trans. 1976, 1424.

(e) Tarassoli, A.; Haltiwanger, R. C.; Norman, A. D. Inorg. Chem. 1982, 21, 2684.

(f) Wiegrabe, W.; Bock, H. Chem. Ber. 1968, 101, 1414

[25] Elowe, P. R.; McCann. C.; Pringle, P. G.; Spitzmesser, S. K.; Bercaw, J. E. Organometallics 2006, 25, 5255.

[26] Noeth, H.; Meinel, L. Z. Anorg. Allg. Chem. 1967, 349, 225.

[27] Blann, K.; Bollmann, A.; Debod, H.; Dixon, J.; Killian, E.; Nongodlwana, P.; Maumela, M.; Maumela, H.; McConnell, A.; Morgan, D. J. Catal. 2007, 249, 244.

[28] Bulloch, G.; Keat, R. J. Chem. Soc., Dalton Trans. 1974, 2010.

[29] Kamalesh Babu, R. P.; Krishnamurthy, S. S.; Nethaji, M. Heteroat. Chem. 1991, 2, 447.

[30] Kamalesh Babu, R. P.; Krishnamurthy, S. S. J. Organomet. Chem. 1993, 454, 157.

[31] Maumela, M.; Blann, K.; de Bod, H.; Dixon, J.; Gabrielli, W.; Williams, D. B. Synthesis 2007, 3863.

[32] Zhao, F. F.; Scopelliti, R.; Dyson, P. J. Dalton Trans. 2003, 2772.

[33] Fenske, D.; Maczek, B.; Maczek, K. Z. Anorg. Allg. Chem. 1997, 623,1113

[34] Schofer, S. J.; Day, M. W.; Henling, L. M.; Labinger, J. A.; Bercaw, J. E. Organometallics 2006, 25, 2743.

[35] Agapie, T.; Day, M. W.; Henling, L. M.; Labinger, J. A.; Bercaw, J. E. Organometallics 2006, 25, 2733.

[36] Bowen, L. E.; Haddow, M. F.; Orpen, A. G.; Wass, D. F. Dalton Trans. 2007, 1160.

[37] Chen, E. Y.; Marks, T. J. Chem. Rev. 2000, 100, 1391.

[38] (a) Blann, K.; Bollmann, A.; Dixon, J. T.; Hess, F. M.; Killian, E.; Maumela, H.; Morgan, D. H.; Neveling, A.; Otto, S.; Overett, M. J. Chem. Commun. 2005, 620.

(b) Blann, K.; Bollmann, A.; Debod, H.; Dixon, J.; Killian, E.; Nongodlwana, P.; Maumela, M.; Maumela, H.; McConnell, A.; Morgan, D. J. Catal. 2007, 249, 244.

[39] Jabri, A.; Crewdson, P.; Gambarotta, S.; Korobkov, I.; Duchateau, R. Organometallics 2006, 25, 715.

[40] Jiang, T.; Liu, X. Y.; Ning, Y. N.; Chen, H. X.; Luo, M. J.; Wang, L. B.; Huang, Z. J. Catal. Commun. 2007, 8, 1145.

[41] McGuinness, D. S.; Overett, M.; Tooze, R. P.; Blann, K.; Dixon, J. T.; Slawin, A. M. Z. Organometallics 2007, 26, 1108.

[42] Rucklidge, A. J.; McGuinness, D. S.; Tooze, R. P.; Slawin, A. M. Z.; Pelletier, J. D. A.; Hanton, M. J.; Webb, P. B. Organometallics
2007, 26, 2782.

[43] Kuhlmann, S.; Blann, K.; Bollmann, A.; Dixon, J.; Killian, E.; Maumela, M.; Maumela, H.; Morgan, D.; Pretorius, M.; Taccardi, N.; Wasserscheid, P. J. Catal. 2007, 245, 279.

[44] Chen, H. X.; Liu, X. Y.; Hu, W. B.; Ning, Y. N.; Jiang, T. J. Mol. Catal. A: Chem. 2007, 270, 273.

[45] Balakrishna, M. S.; Reddy, V. S.; Krishnamurthy, S. S.; Nixon, J. F.; Laurent, J. C. Coord. Chem. Rev. 1994, 129, 1.

[46] Cloete, N.; Visser, H. G.; Engelbrecht, I.; Overett, M. J.; Gabrielli, W. F.; Roodt, A. Inorg. Chem. 2013, 52, 2268.

[47] (a) Schulz, G. V. Z. Phys. Chem., Abt. B 1935, 30, 379.

(b) Schulz, G. V. Z. Phys. Chem., Abt. B 1939, 43, 25.

(c) Flory, P. J. J. Am. Chem. Soc. 1940, 62, 1561.

[48] (a) Forestiere, A.; Olivier-Bourbigou, H.; Saussine, L. Oil Gas Sci. Technol. Rev. IFP 2009, 64, 649.

(b) Al-Sa'doun, A. W. Appl. Catal., A 1993, 105, 1.

(c) Commereuc, D.; Chauvin, Y.; Gaillard, J.; Leonard, J.; Andrews, J. Hydrocarbon Process 1984, 63, 118.

[49] (a) Belov, G. P.; Dzhabiev, T. S.; Kolesnikov, I. M. J. Mol. Catal. 1982, 14, 105.

(b) Agapie, T.; Schofer, S. J.; Labinger, J. A.; Bercaw, J. E. J. Am. Chem. Soc. 2004, 126, 1304.

[50] (a) Spencer, M. D.; Morse, P. M.; Wilson, S. R.; Girolami, G. S. J. Am. Chem. Soc. 1993, 115, 2057.

(b) You, Y.; Girolami, G. S. Organometallics 2008, 27, 3172.

[51] [51] Manyik, R. M.; Walker, W. E.; Wilson, T. P. J. Catal. 1977, 47, 197.

[52] (a) McDermott, J. X.; White, J. F.; Whitesides, G. M. J. Am. Chem. Soc. 1973, 95, 4451.

(b) McDermott, J. X.; White, J. F.; Whitesides, G. M. J. Am. Chem. Soc. 1976, 98, 6521.

[53] (a) Theopold, K. H. Eur. J. Inorg. Chem. 1998, 15. (b) McDaniel, M. P. Adv. Catal. 1985, 33, 47.

[54] Briggs, J. R. Chem. Commun. 1989, 11, 674.

[55] (a) Emrich, R.; Heinemann, O.; Jolly, P. W.; Kruger, C.; Verhovnik, G. P. Organometallics 1997, 16, 1511.

(b) Freeman, J. W.; Buster, J. L.; Knudsen, R. D. US 5856257, 1999, [Chem. Abstr. 1999, 130, 415984].

[56] Overett, M. J.; Blann, K.; Bollmann, A.; Dixon, J. T.; Haasbroek, D.; Killian, E.; Maumela, H.; McGuinness, D. S.; Morgan, D. H. J. Am. Chem. Soc. 2005, 127, 10723.

[57] (a) Yu, Z.; Houk, K. N. Angew. Chem. Int. Ed. 2003, 42, 808. (b) Blok, A. N. J.; Budzelaar, P. H. M.; Gal, A. W. Organometallics 2003, 22, 2564.

[58] Tobisch, S.; Ziegler, T. Organometallics 2003, 22, 5392.

[59] Wang, J. J.; An, D. L.; Zhu, H. P. Chin. J. Struct. Chem. 2010, 29, 933.

[60] Morgan, D. H.; Schwikkard, S. L.; Dixon, J. T.; Nair, J. J.; Hunter, R. Adv. Synth. Catal. 2003, 345, 939.

[61] van Rensburg, W. J.; Grove, C.; Steynberg, J. P.; Stark, K. B.; Huyser, J. J.; Steynberg, P. J. Organometallics 2004, 23, 1207.

[62] Walsh, R.; Morgan, D. H.; Bollmann, A.; Dixon, J. T. Appl. Catal. A 2006, 306, 184.

[63] Yang, Y.; Kim., H. J.; Lee, J.; Paikb, H.; Jang, H. G. Appl. Catal., A 2000, 193, 29.

[64] Reagen, W. K.; Conroy, B. K. CA 2020509, 1991 [Chem. Abstr. 1992, 116, 60189].

[65] Overett, M. J.; Blann, K. .; Bollmann, A.; Dixon, J. T.; Hess, F.; Killian, E.; Maumela, H.; Morgan, D. H.; Neveling, A.; Otto, S. Chem. Commun. 2005, 622.

[66] Blann, K.; Bollmann, A.; Dixon, J. T.; Neveling, A.; Morgan, D. H.; Maumela, H.; Killian, E.; Hess, F. M.; Otto, S.; Pepler, L.; 
Mahomed, H. A.; Overett, M. J.; WO 2004/056479, 2002 [Chem. Abstr. 2004, 141, 89536].

[67] Kuhlmann, S.; Dixon, J. T.; Haumann, M.; Morgan, D. H.; Ofili, J.; Spuhl, O.; Taccardi, N.; Wasserscheid, P. Adv. Synth. Catal. 2006, $348,1200$.

[68] Jiang, T.; Ning, Y. N.; Zhang, B. J.; Li, J. Z.; Wang, G.; Yi, J. J.; Huang, Q. J. Mol. Catal. A: Chem. 2006, 259, 161.

[69] Paul R.; Elowe, C. M.; Pringle, P. G.; Spitzmesser, S. K.; Bercaw, J. E. Organometallics 2006, 25, 5255.

[70] Killian, E.; Blann, K.; Bollmann, A.; Dixon, J. T.; Kuhlmann, S.; Maumela, M. C.; Maumela, H.; Morgan, D. H.; Nongodlwana, P.; Overett, M. J.; Pretorius, M.; Höfener, K.; Wasserscheid, P. J. Mol.
Catal. A: Chem. 2007, 270, 214

[71] McGuinness, D. S.; Rucklidge, A. J.; Tooze, R. P.; Slawin, A. M. Z. Organometallics 2007, 26, 2561.

[72] Weng, Z. q.; Teo, S. h.; Andy Hor, T. S. Dalton Trans. 2007, 3493.

[73] Jiang, T.; Zhang, S.; Jiang, X. L.; Yang, C. F.; Niu, B.; Ning, Y. N. J. Mol. Catal. A: Chem. 2008, 279, 90.

[74] Jiang, T.; Tao, Y. Q.; Gao, X. L.; Mao, G. L.; Chen, H. X.; Chen, C. G. .; Ning, Y. N. Chin. Sci. Bull. 2012, 57, 1510.

[75] Sa, S.; Lee, S. M.; Kim, S. Y. J. Mol. Catal. A: Chem. 2013, 378, 17.

[76] Shao, H.; Li, Y.; Gao, X.; Cao, C.; Tao, Y.; Lin, J.; Jiang, T. J. Mol. Catal. A: Chem. 2014, 390, 152. 